\title{
Carex flava agg. (section Ceratocystis, Cyperaceae) in Poland: distribution maps and locality lists
}

\author{
Helena Więcław
}

Department of Plant Taxonomy and Phytogeography, Faculty of Biology, University of Szczecin, Wąska 13, 71-415 Szczecin, Poland, e-mail: wieclawh@univ.szczecin.pl

\begin{abstract}
This paper presents lists of localities and distribution maps of Carex taxa of the section Ceratocystis in Poland. Distribution maps of individual taxa are based on the ATPOL grid, using material deposited in Polish herbaria and private collections as well as material collected during field research in 2007-2012. The collected data indicate that (i) in Poland C. flava s.s. is the most common species of the section Ceratocystis, whereas C. hostiana is the rarest one; (ii) most of the localities of C. demissa are situated in southern Poland, especially in Małopolska, Podkarpacie, and Silesia Provinces (iii) C. lepidocarpa is most frequent in Polesie, Lublin Upland, Małopolska, and Pomerania; no records of this species in southernmost parts of Poland have been confirmed; (iv) C. viridula var. viridula is frequent in Poland, whereas $C$. viridula var. pulchella is only known from 2 localities in the north; (v) the most frequent hybrid in Poland is $C . \times$ alsatica [C. demissa $\times C$. flava], while C. $\times$ ruedtii $[$ C. flava $\times$ C. lepidocarpa], C. $\times$ subviridula $[$ C. flava $\times$ C. viridula], and C. $\times$ schatzii $[$ C. lepidocarpa $\times$ C. viridula $]$ are reported less often; the rarest hybrids are $C$. demissa $\times C$. viridula, $C . \times f u l v a[C$. demissa $\times C$. hostiana], and $C . \times l e u t z i i$ [C. hostiana $\times$ C. lepidocarpa].
\end{abstract}

Key words: Carex flava agg., Carex hostiana, hybrids, Ceratocystis, Cyperaceae, distribution, ATPOL, Poland

\section{Contents}

1. Introduction

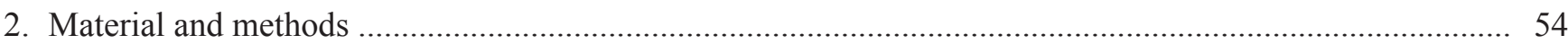

3. Distribution maps of Carex taxa within the section Ceratocystis .......................................................... 55

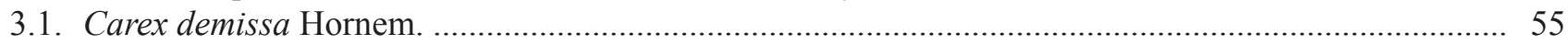

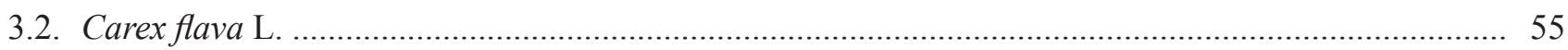

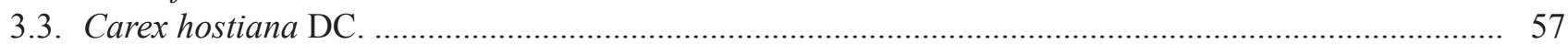

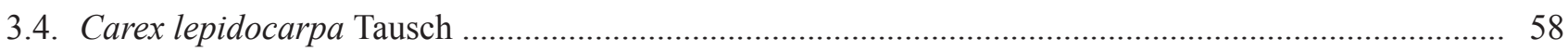

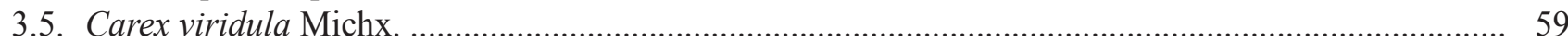

4. Distribution maps of hybrids within the section Ceratocystis ................................................................. 59

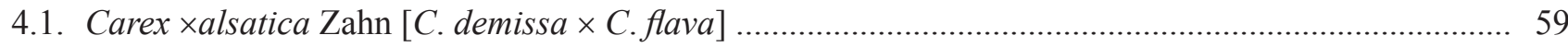

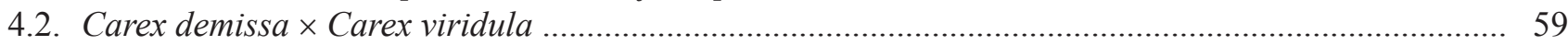

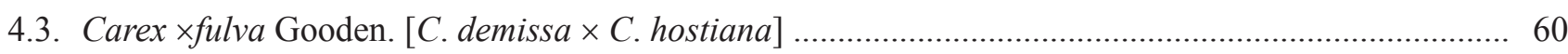

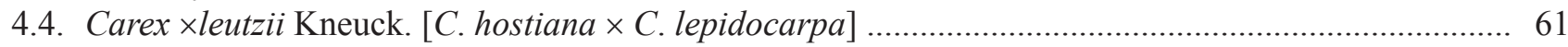

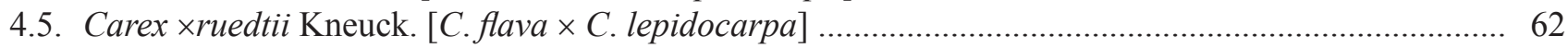

4.6. Carex $\times$ schatzii Kneuck. [C. lepidocarpa $\times$ C. viridula] ................................................................ 63

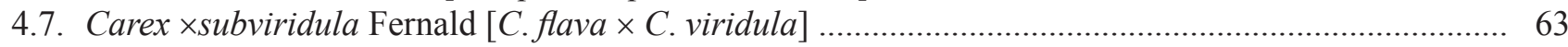

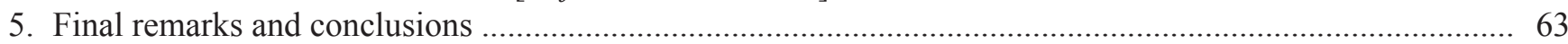

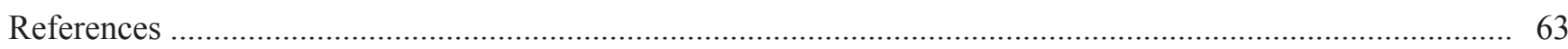

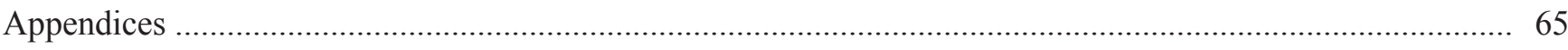




\section{Introduction}

The genus Carex L. is one of the few truly cosmopolitan plant genera (Good 1974) with centres of diversity in the temperate regions of Asia, Europe, and the Americas (Govaerts et al. 2010). Sedges of this genus are absent in tropical lowlands, except for a few species present in southeast Asia and sub-Saharan Africa (Ball \& Reznicek 2002). Some species exhibit disjunct distribution patterns, such as bipolar or eastern Asianeastern North American distributions (Moore \& Chater 1971; Ball 1990; Escudero et al. 2010; Villaverde et al. 2012).

Taxa of the section Ceratocystis Dumort. are found frequently in the Northern Hemisphere, frequent both in Europe and in North America (Meusel et al. 1965; Hultén \& Fries 1986a, 1986b). Members of this section were described by Raymond (1951) as Amphi-Atlantic species (see also Davies 1953b), but according to Ball (1990), species like C. flava L. and C. viridula Michx., which are more widespread in Europe and North America, but absent from much of Asia, are probably more reasonably interpreted as fragmented circumpolar distribution (see also Kuchel \& Bruederle 2000).

In Poland 6 taxa of the section Ceratocystis are found: C. hostiana DC., C. flava L., C. lepidocarpa Tausch, C. demissa Hornem., C. viridula Michx. var. viridula, and var. pulchella (Lönnroth) B. Schmid, and the following 7 hybrids: $C$. $\times$ alsatica Zahn, $C$. $\times$ ruedtii Kneuck., C. ×schatzii Kneuck., C. ×subviridula Fernald, $C$. demissa $\times C$. viridula, $C . \times$ fulva Gooden., and C. xleutzii Kneuck. (Więcław 2014). Among them, C. hostiana and C. flava are morphologically best defined, usually easily identifiable. Delimitation of other taxa of the section Ceratocystis is difficult because of their variable morphology, overlap of ranges of many characters, and appearance of hybrids whose characters are intermediate between those of parental species (Więcław 2014).

During examination of herbarium specimens, quite some errors in species identification were noticed on their labels. Some herbarium specimens were hybrids classified by collectors as pure species (in fact, hybrids within the section Ceratocystis were not distinguished in Poland before this study). The complex pattern of variation of taxa within the section Ceratocystis makes it difficult to delimit them unambiguously (which was evidenced empirically during examination of herbarium specimens). Thus it can be supposed that some published data about their distribution in Poland are probably also wrong and do not reflect their true distribution. That is why this study does not take into account localities known only from the literature, whose identification could not be verified because no herbarium specimens are available.
This study was aimed to present the distribution of species and hybrids within the section Ceratocystis in Poland based on herbarium specimens loaned from Polish herbaria and private collections as well as on material collected during my field research.

\section{Material and methods}

Plant material for this study included herbarium specimens from 26 Polish herbaria (BIL, BNPH, BSG, BYDG, DRAPN, KRA, KRAM, KRAB, KTC, KTU, LBLM, LOD, OLTC, OPOL, POZ, PUMA, TRN, SLTC, SPNH, SZUB, SZCZ, UGDA, WA, WRSL, WSRP, ZAMU) and loans from 7 private collections, as well as plants collected during field research in 2007-2012. Abbreviations of the names of herbaria follow Mirek et al. (1997).

Localities of pure taxa and hybrids are presented on maps based on the ATPOL grid (Zając 1978) and as locality lists ordered alphabetically according to Polish names of provinces (voivodeships): Dolnośląskie (Lower Silesia Province), Kujawsko-Pomorskie (Kujawy-Pomerania Province), Lubelskie (Lublin Province), Lubuskie (Lubusz Province), Łódzkie (Łódź Province), Małopolskie (Małopolska [Lesser Poland] Province), Mazowieckie (Masovia Province), Opolskie (Opole Province), Podkarpackie (Podkarpacie Province), Podlaskie (Podlasie Province), Pomorskie (Pomerania Province), Śląskie (Silesia Province), Świętokrzyskie (Świętokrzyskie Province), WarmińskoMazurskie (Warmia-Masuria Province), Wielkopolskie (Wielkopolska [Greater Poland] Province), Zachodniopomorskie (West Pomerania Province).

The records include the following data: ATPOL square, place of collection (geographic name of the locality: town, village, lake, nature reserve, forest section, etc., depending on information given on labels), collector's name, year of collection, herbarium name abbreviation (private collections and own material were marked with * and ${ }^{* *}$, respectively). Taxa are listed alphabetically, first the species and lower taxa, followed by the hybrids.

In many cases (most of the specimens collected in the late $19^{\text {th }}$ and early $20^{\text {th }}$ century), labels of herbarium specimens contained too general information about the location of the taxa; often the geographic name was ambiguous (as localities with the same name are found in various parts of Poland) or no information was given about place of collection. In such cases, the ATPOL square was not specified but the information was used in general descriptions of distribution of individual taxa. The following symbols were used on maps to distinguish between localities, depending on the year of record/collection of individual specimens: ( $\mathbf{-}$ - no date, $\times$ - sedges collected till 1960, - - sedges collected in 1961-1990, - - sedges collected in 19912013. 


\section{Distribution maps of Carex taxa within the section Ceratocystis}

\subsection{Carex demissa Hornem.}

Amphi-Atlantic species (Zając \& Zając 2009), distributed in northern, western, and central Europe, north-eastern Africa, and western part of North America (Hultén \& Fries 1986a, 1986b). It has not been recorded in Russia (Egorova 1999), while in North America it is probably an adventive species (Crins \& Ball 1989). From Iran and Afghanistan, Kukkonen (1984) has been described C. demissa subsp. iranica Kukkonen.

In Poland C. demissa was first noted in the Bieszczady Mts in 1965, near the village of Baligród (Jasiewicz 1965). Currently the species is found in scattered localities in all provinces of Poland, in 176 ATPOL squares (Fig. 1, Appendix 1). It is most common in southern Poland, especially in Małopolska, Podkarpacie, and Silesia Provinces (Małopolskie, Podkarpackie, and Śląskie, respectively). The species is frequent in the mountains and in the submontane zone, but scattered in lowlands. The most elevated locality, about $1290 \mathrm{~m}$ a.s.l., is known from the Żywiec Beskids (Beskid Żywiecki Mts) near Hala Miziowa (see Appendix 1). Similarly, in Bohemia (within the former Czechoslovakia), C. demissa is quite frequent in the foothills and mountains (Havlícková 1983). In the British Isles it is the most common species of the section Ceratocystis (Davies 1953b, 1956).

Carex demissa usually grows in meadows, on roadsides, in fens and intermediate mires, groundwater seepage areas, pastures, in moist depressions, moist fallows, forests (spruce, fir-spruce, fir-beech, alluvial or oak-hornbeam), in forest glades and roadsides, near ponds, ditches, along streams and rivers.

\subsection{Carex flava $\mathrm{L}$.}

Circum-Boreal species (Zając \& Zając 2009), distributed nearly all over Europe, in $\mathrm{W}$ and $\mathrm{E}$ parts of North America, and in North Africa; in Asia recorded as a relict species, mostly in the Caucasus and south of the Caspian Sea (Meusel et al. 1965; Hultén \& Fries 1986a, 1986b).

Carex flava is the most common species of the section Ceratocystis in Poland. The collected material documents its occurrence in 571 ATPOL squares (Fig. 2, Appendix 1), and the resultant map is generally similar to the map presented in the atlas of distribution of vasular plants in Poland (Zając \& Zając 2001). This sedge species is more frequent in mountains and in the submontane zone than in lowlands. The most elevated locality, about $1650 \mathrm{~m}$ a.s.1., is known from the Tatra Mts (Mały Kopieniec and slope of Miedziane; see Appendix 1). More of its localities are situated in eastern

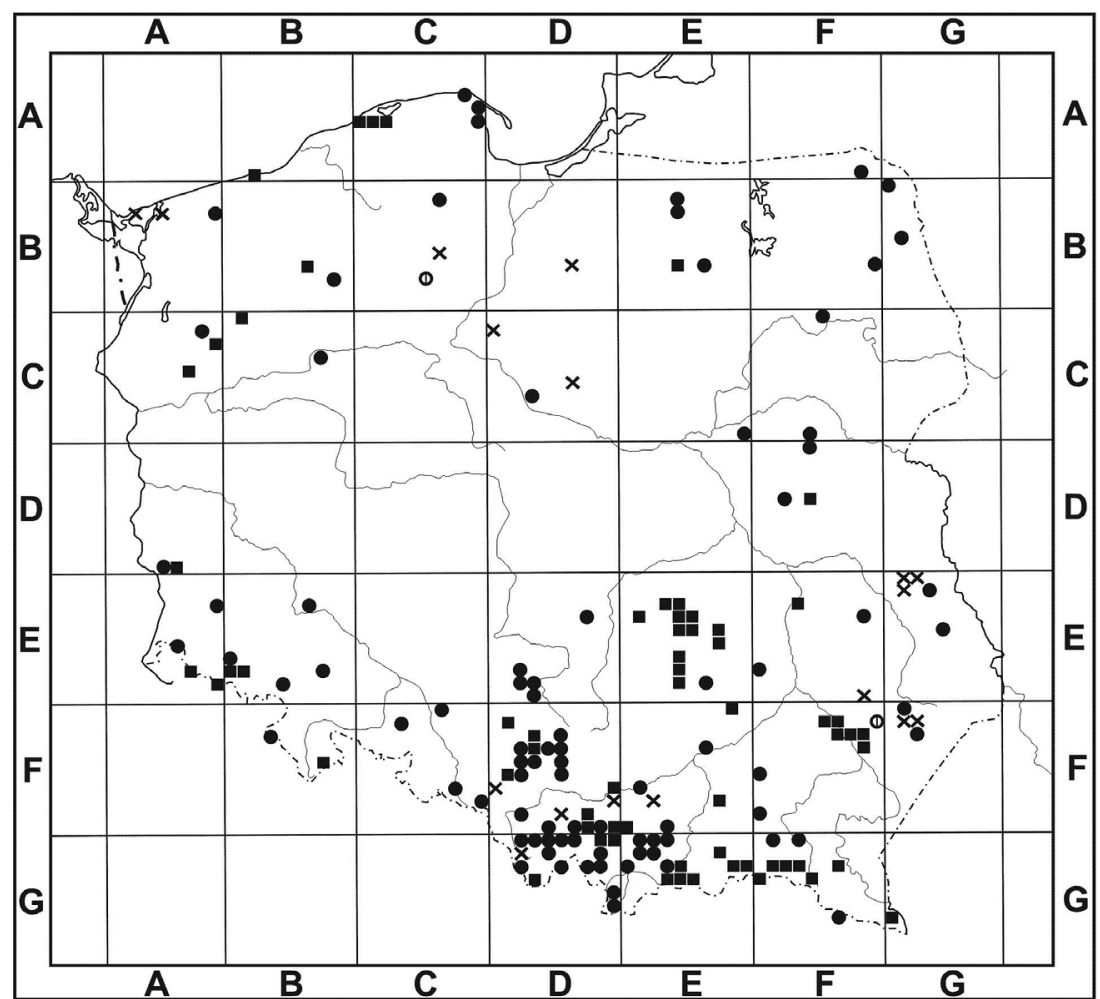

Fig. 1. Distribution map of Carex demissa Hornem.

Explanations: (1) - no date, $\times$ - sedges collected till 1960 , 


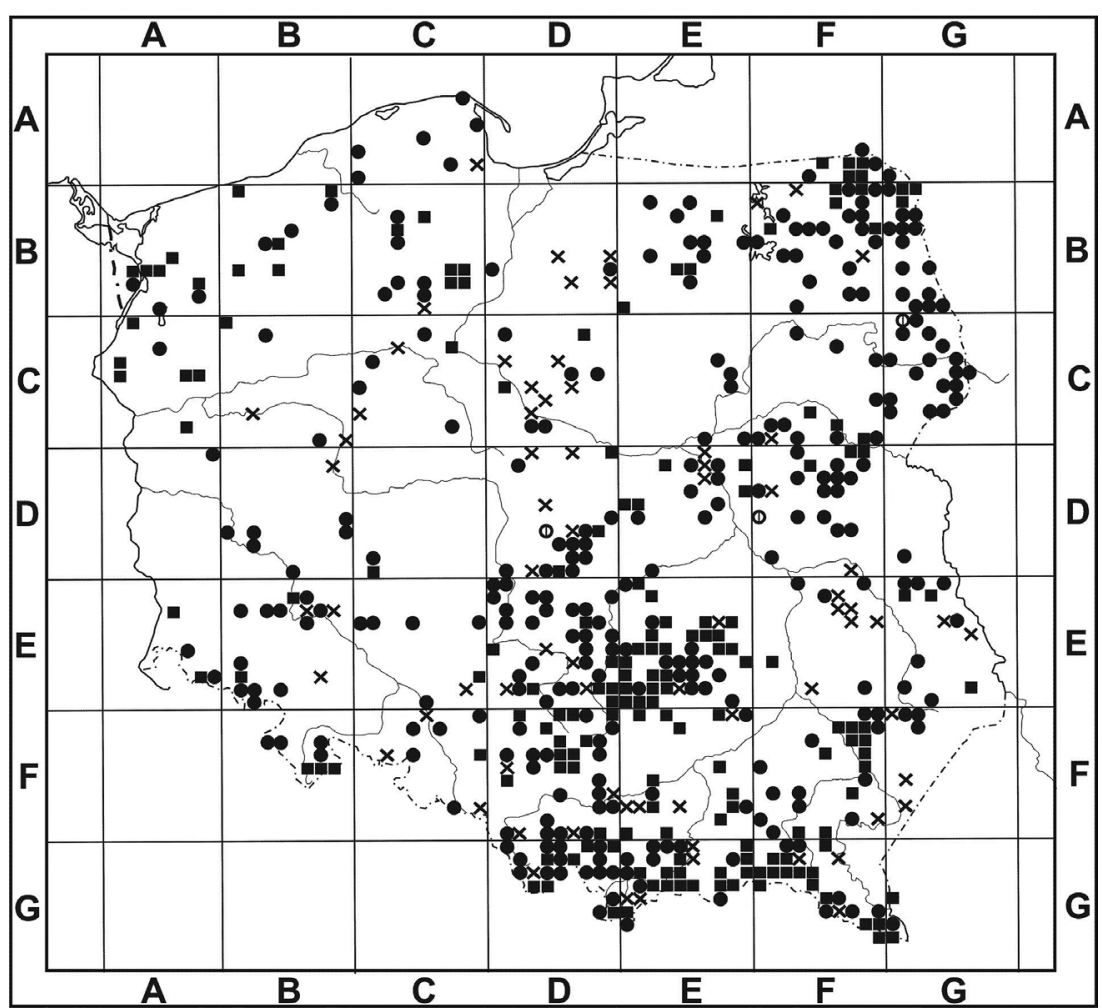

Fig. 2. Distribution map of Carex flava $\mathrm{L}$.

Explanations: $\mathbb{D}$ - no date, $\times$ - sedges collected till 1960, - - sedges collected in 1961-1990, $\mathbf{\square}$ - sedges collected in 1991-2013

and southern Poland than in its western and northern parts.

In Scandinavia, Switzerland, the Czech Republic, and Bulgaria, C. flava is common (Schmid 1981; Hav- lícková 1983; Stoeva \& Štepánková 1990; Pykälä \& Toivonen 1994), but it is very rare in the British Isles (Davies 1953a, 1953b, 1956; Blackstock \& Ashton 2001).

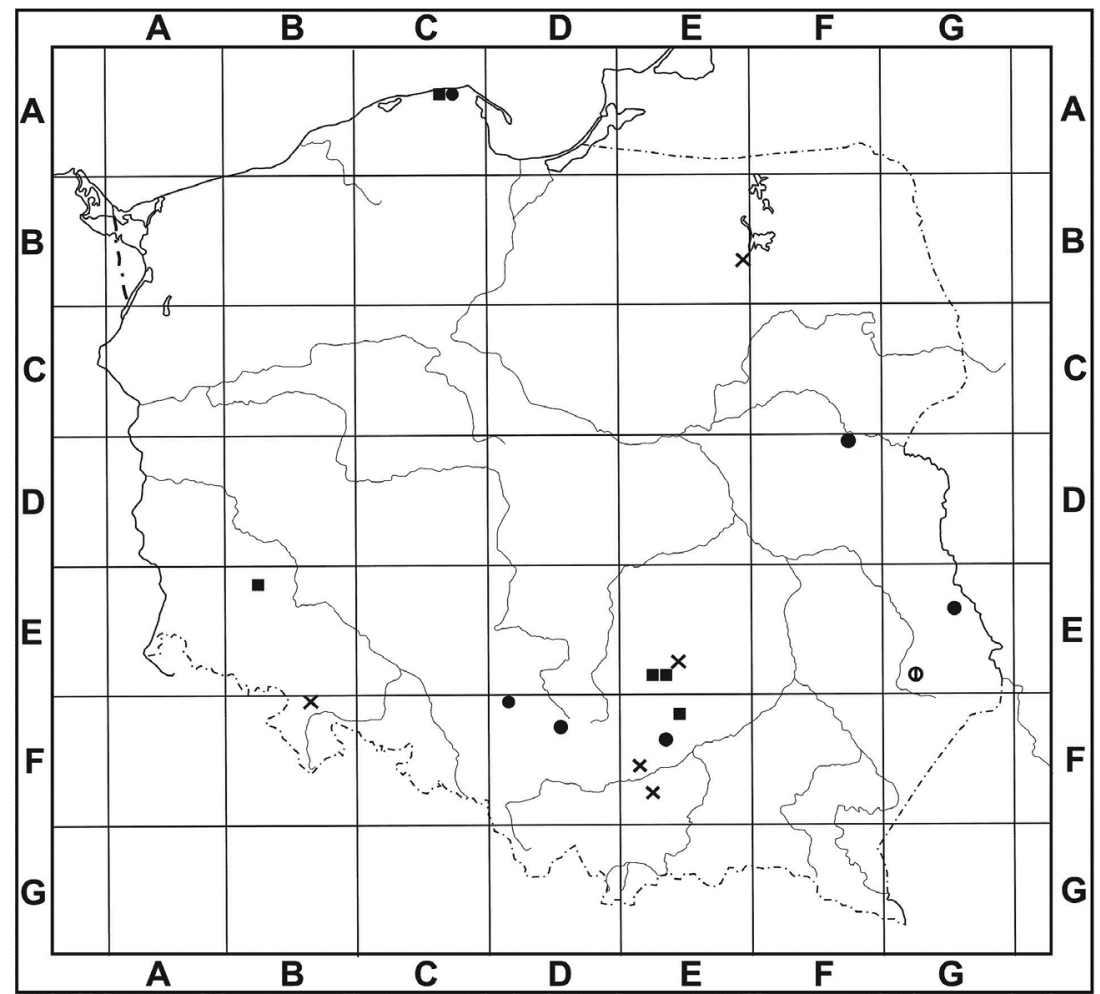

Fig. 3. Distribution map of Carex hostiana DC. 
This sedge species usually grows in moist meadows, marshes, peatlands, ditches, along streams, in roadside depressions, along forest roads, on pastures, in thickets, at forest edges, and in forests (alluvial, wet coniferous, pine, fir-spruce, or alder).

\subsection{Carex hostiana DC.}

European-temperate species of lowland group with Balkan-type distribution, associated especially with Atlantic part of Europe (Zając \& Zając 2009). Distributed nearly all over Europe (except eastern part), rarely in the Mediterranean region (Meusel et al. 1965; Chater 1980). Reported also from North America, as C. hostiana var. laurentiana Fern \& Wieg. (Raymond 1950; Hultén \& Fries 1986a, 1986b).

In Poland C. hostiana is relatively rare, as it reaches its eastern limit here (Sotek 2010). According to Zając and Zając (2001), it is recorded mostly in the west and southwest of Poland (Silesia and Małopolska). However, results of the present study confirm localities of this sedge only in 17 ATPOL squares in 8 provinces (Fig. 3, Appendix 1). The map of distribution of C. hostiana presented here includes a lower number of localities than the map in the atlas of the Polish vascular flora (Zając \& Zając 2001). This results from 3 factors: (i) Polish herbaria comprise a small number of specimens of $C$. hostiana, $(i i)$ some herbarium specimens were hybrids, $C$. $\times$ fulva $[$ C.demissa $\times C$.hostiana $]$ or $C . \times$ leutzii $[$ C. hostiana $\times$ C. lepidocarpa $]$, instead of C. hostiana, (iii) on some herbarium sheets with specimens collected in the late $19^{\text {th }}$, no information about location was given (4 sheets in TRN herbarium).

Carex hostiana is found in moist meadows and fens, usually on more or less calcareous soils.

\subsection{Carex lepidocarpa Tausch}

Amphi-Atlantic species (Zając \& Zając 2009), distributed nearly all over Europe and in western Canada (Meusel et al. 1965; Hultén \& Fries 1986a, b). In many European countries, C. lepidocarpa is declining because of drainage of wetlands (Schmid 1981; Havlícková 1983; Pykälä 1994).

According to Zając and Zając (2001), and Sotek (2010), C. lepidocarpa in frequent in southern Poland, primarily in the Eastern Carpathians and Bieszczady Mts. However, on the basis of this study and revision of herbarium material, the pattern of distribution of this species in Poland seems to be different. During field research in the Bieszczady Mts, no local population of C. lepidocarpa was found. Revision of herbarium specimens also did not confirm frequent occurrence of this species in the south of Poland, since most of the herbarium sheets labelled as C. lepidocarpa contained specimens of C. flava s.s.

Localities of C. lepidocarpa were recorded in 103 ATPOL squares in 14 provinces (Fig. 4, Appendix 1). Its localities are most numerous in Polesie and Lublin Upland, Małopolska, Lithuanian Lakeland, and Western

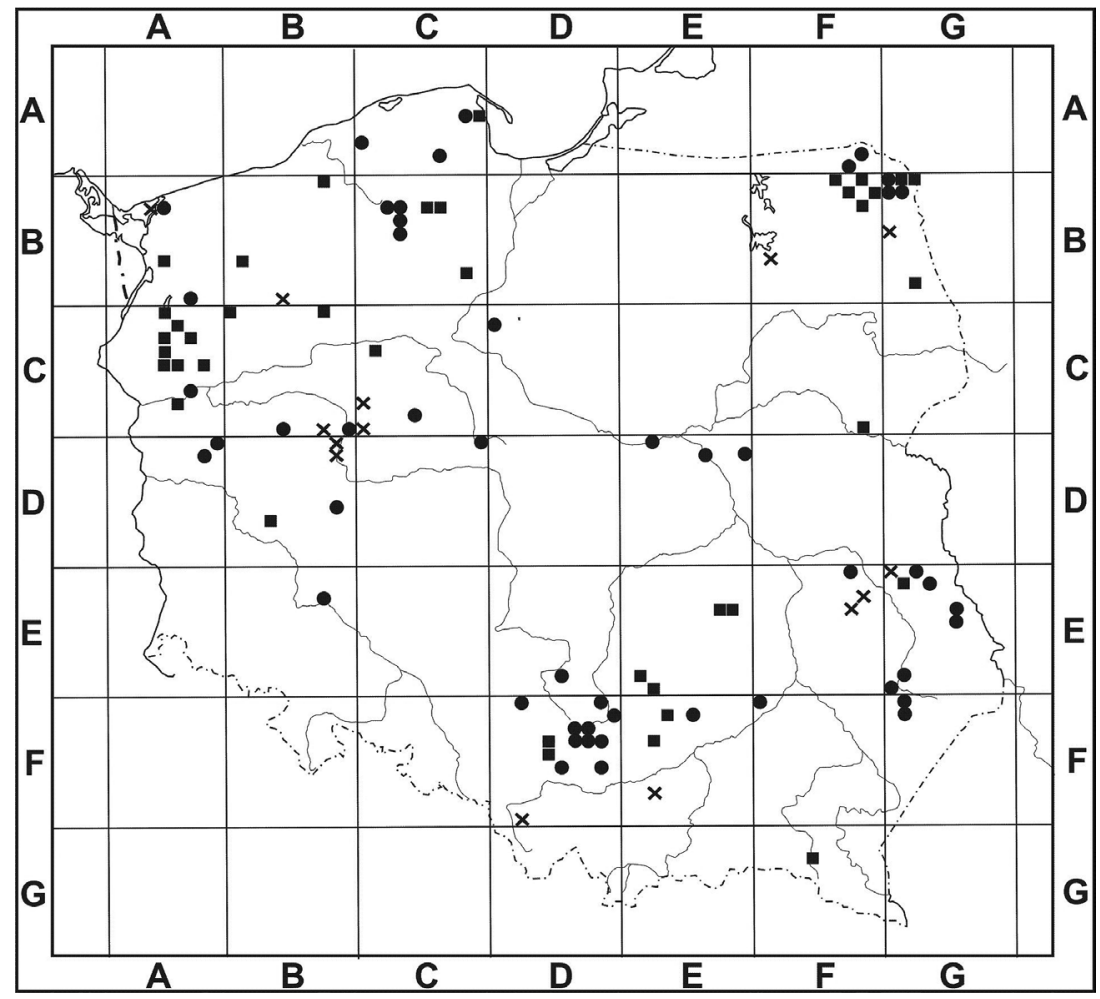

Fig. 4. Distribution map of Carex lepidocarpa Tausch

Explanations: $\times$ - sedges collected till 1960, - sedges collected in 1961-1990, $\mathbf{0}$ - sedges collected in 1991-2013 


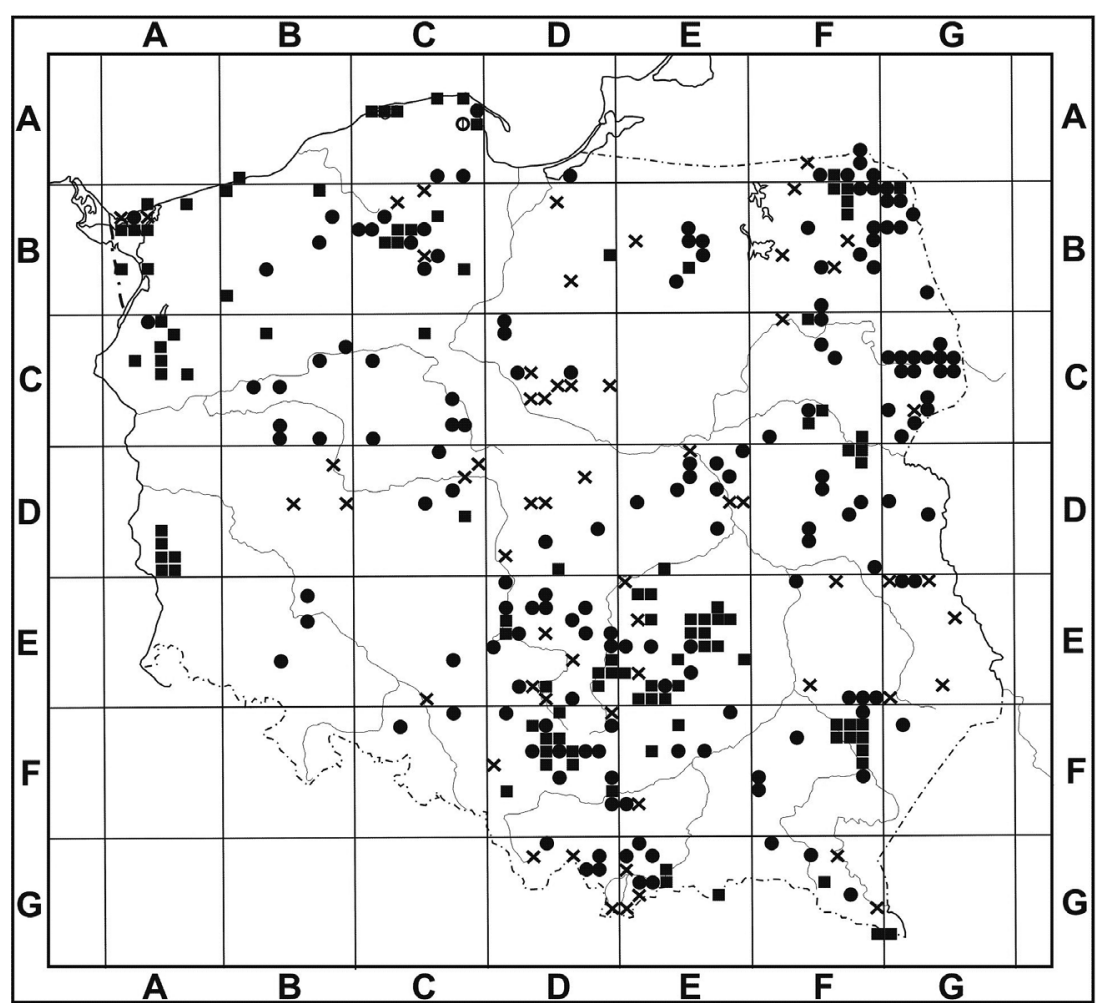

Fig. 5. Distribution map of Carex viridula Michx. var. viridula

Explanations: $\mathbb{D}$ - no date, $\times$ - sedges collected till 1960, - - sedges collected in 1961-1990, $\mathbf{\square}$ - sedges collected in 1991-2013

Pomerania. The species is usually found in plains or on hills. In Poland its most elevated record was at $460 \mathrm{~m}$ a.s.l., near Wernejówka in Podkarpacie Province; simi- larly, in the Czech Republic and Finland, it is usually distributed in plains or on small hills, reaching altitudes of up to about 300 m a.s.l. (Havlícková 1983; Pykälä

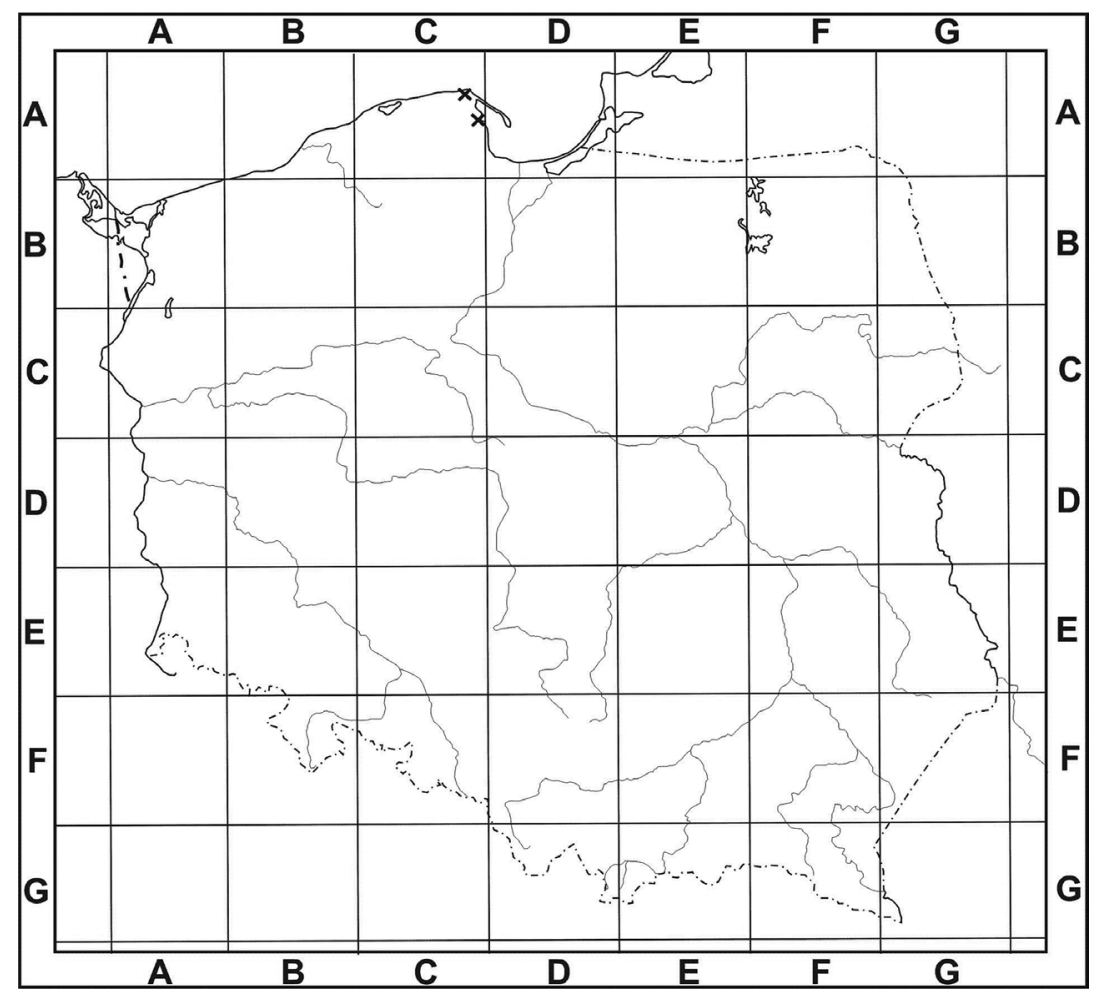

Fig. 6. Distribution map of Carex viridula var. pulchella (Lönnr.) B. Schmid Explanations: $\times-$ sedges collected till 1960 
1994), whereas in Switzerland its most elevated record was at about $2000 \mathrm{~m}$ a.s.1. (Schmid 1981).

Carex lepidocarpa typically grows on calcareous soils, on moist and waterlogged meadows, groundwater seepage areas, typical and poor fens.

\subsection{Carex viridula Michx. (var. viridula and var. pulchella)}

Circum-Boreal species with disjunctive range (Zając \& Zając 2009), distributed nearly all over Europe (rarer in the Mediterranean region), in northern and central parts of North America and in western Asia (Chater 1980; Hultén \& Fries 1986a, b). The common variety, C. viridula var. viridula, is found nearly throughout the range of distribution of this species, whereas $C$. viridula var. pulchella is reported from Scandinavia (Pykälä \& Toivonen 1994), Russia (Egorova 1999), Scotland (Davies 1953a), the Netherlands (Kern \& Reichelt 1954), Germany (Schulze-Motel 1980), and Poland (Zając 1968).

Carex viridula var. viridula is frequent in Poland, recorded in lowlands and in mountains; its most elevated locality (about $1187 \mathrm{~m}$ a.s.l.) is situated in Małopolska Province (eastern peak of Łysanki, Western Tatras). According to Zając and Zając (2001), localities of this sedge are most numerous in the South Podlasie Lowland (Nizina Południowopodlaska) and Lublin Upland. Results of this study indicate that this sedge species is relatively frequent in northern, central, and south- eastern Poland, whereas in the south-western part its localities are scattered; recorded in 336 ATPOL squares (Fig. 5, Appendix 1).

Carex viridula var. viridula grows in moist meadows, marshes, groundwater seepage areas, typical, poor, and calcareous fens, sandy depressions at edges of lakes, ponds, waterholes, streams, ditches, on roadsides, in salt-marshes, and thickets.

Carex viridula var. pulchella is very rare; recorded in only 2 ATPOL squares in Pomerania province (Fig. 6).

\section{Distribution maps of hybrids within the section Ceratocystis}

\subsection{Carex $\times$ alsatica Zahn $[$ C. demissa $\times$ C.flava $]$}

Hybrid reported mostly from northern and western Europe (Koopman 2011). In Poland it is the most frequent hybrid of the C. flava complex; recorded in 44 ATPOL squares in 12 provinces (Fig. 7, Appendix 2).

\subsection{Carex demissa $\times$ Carex viridula}

In Europe rare, known from Scandinavia, the Netherlands, Germany, Great Britain, Spain, Slovakia, Latvia, and Estonia (Koopman 2011). In Poland found in 2 ATPOL squares in 2 provinces (Fig. 8, Appendix 2).

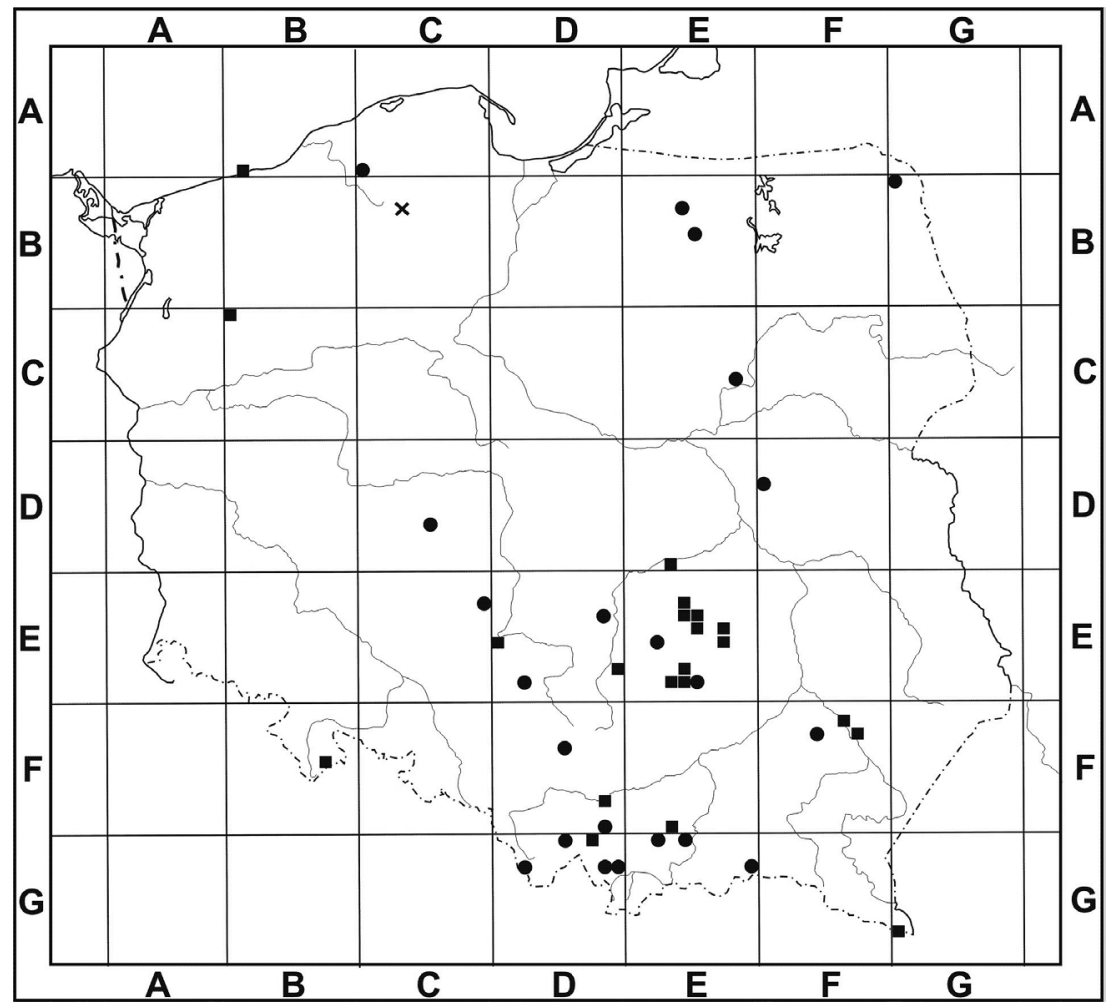

Fig. 7. Distribution map of Carex $\times$ alsatica Zahn

Explanations: $\times$ - sedges collected till 1960, $\bullet$ - sedges collected in 1961-1990, - sedges collected in 1991-2013 


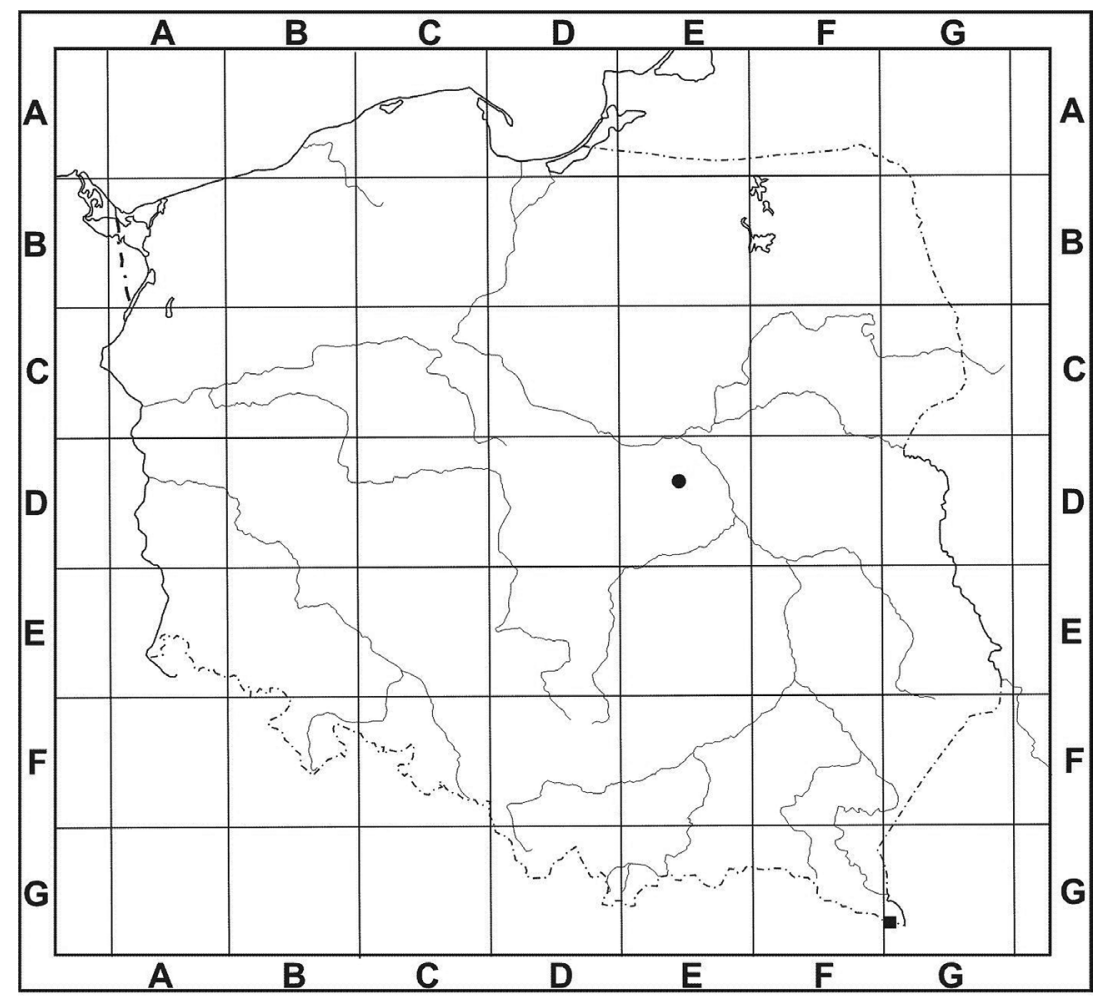

Fig. 8. Distribution map of Carex demissa $\times$ Carex viridula

Explanations: - - sedges collected in 1961-1990, $\mathbf{\square}$ - sedges collected in 1991-2013

\subsection{Carex $\times$ fulva Gooden. [C. demissa $\times$ C. hostiana $]$}

Hybrid quite frequent in Europe (Kiffe 2001; Koopman 2011; Więcław \& Koopman 2013). In Poland, localities of $C$. $\times$ fulva have been recorded in the north, in 3 ATPOL squares in 2 provinces (Fig. 9, Appendix 2). Specimens of this hybrid were found only in herbaria (OLTC, TRN).

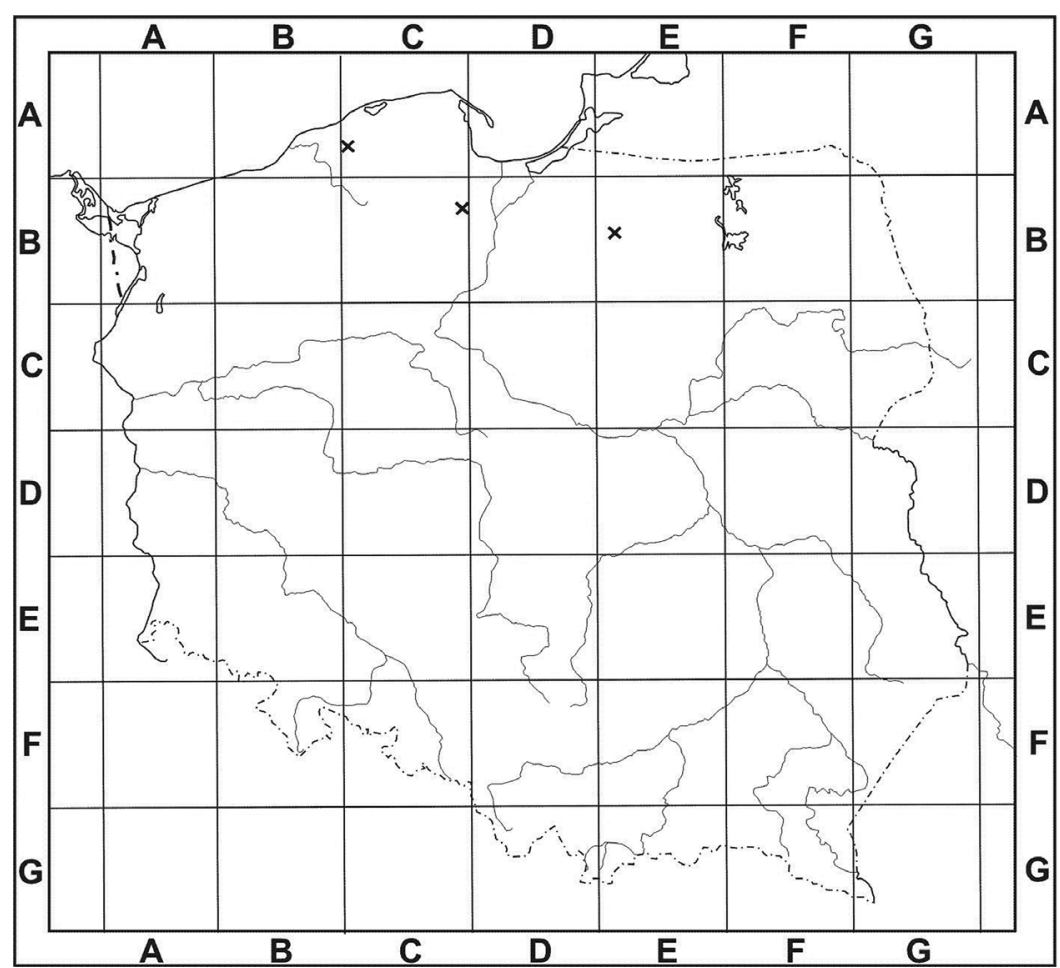

Fig. 9. Distribution map of Carex $\times$ fulva Gooden.

Explanations: $\times-$ sedges collected till 1960 


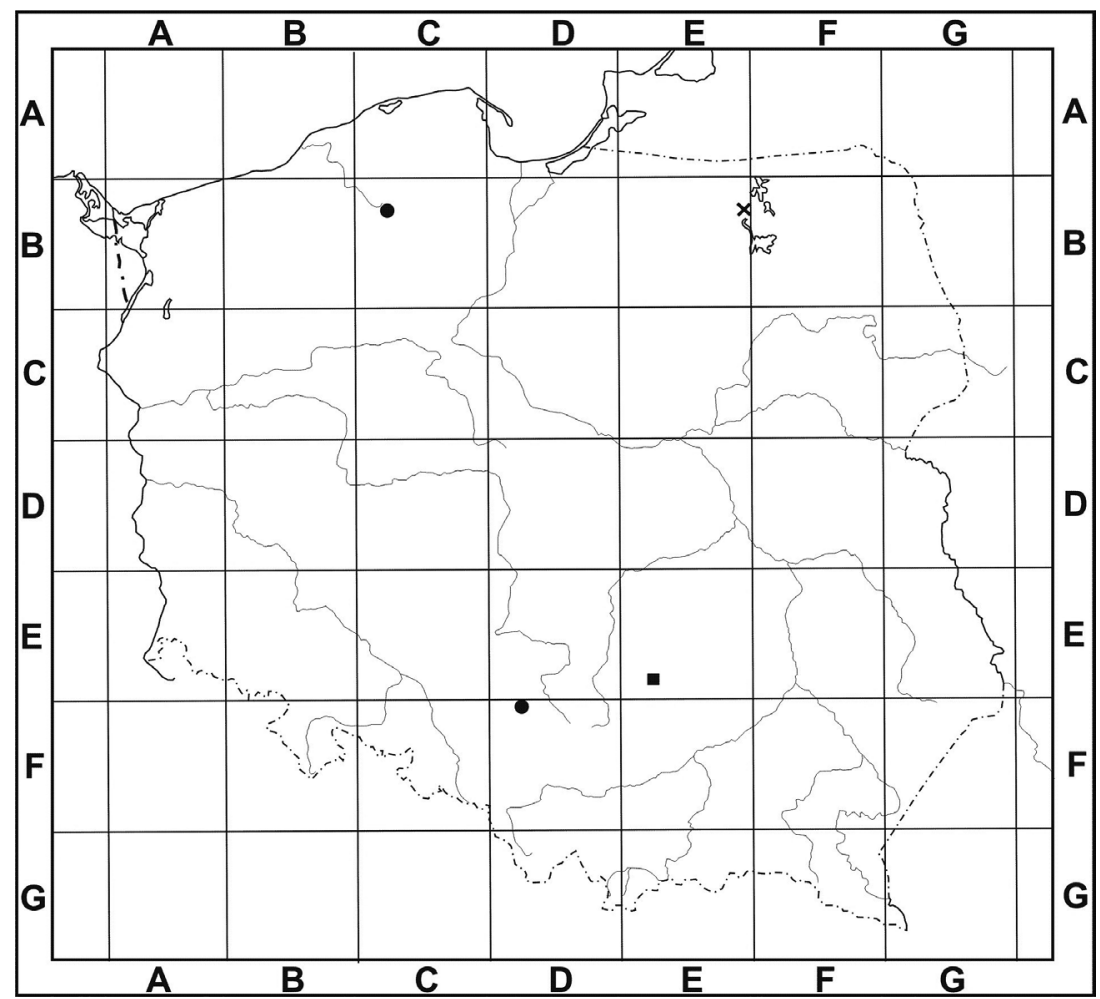

Fig. 10. Distribution map of Carex $\times$ leutzii Kneuck.

Explanations: $\times$ - sedges collected till 1960, - sedges collected in 1961-1990,

- sedges collected in 1991-2013

\subsection{Carex $\times$ leutzii Kneuck. [C. hostiana $\times$ C. lepido- carpa]}

Hybrid reported from Scandinavia, Great Britain, Denmark, Belgium, Germany, Switzerland, Italy,
Austria, Slovakia, Lithuania, Latvia, and Estonia (Kiffe 2001; Koopman 2011; Więcław \& Koopman 2013). In Poland recorded in 4 ATPOL squares in 4 provinces (Fig. 10, Appendix 2). Specimens of $C$. ×leutzii were found only in herbaria (KRA, LOD, POZ).

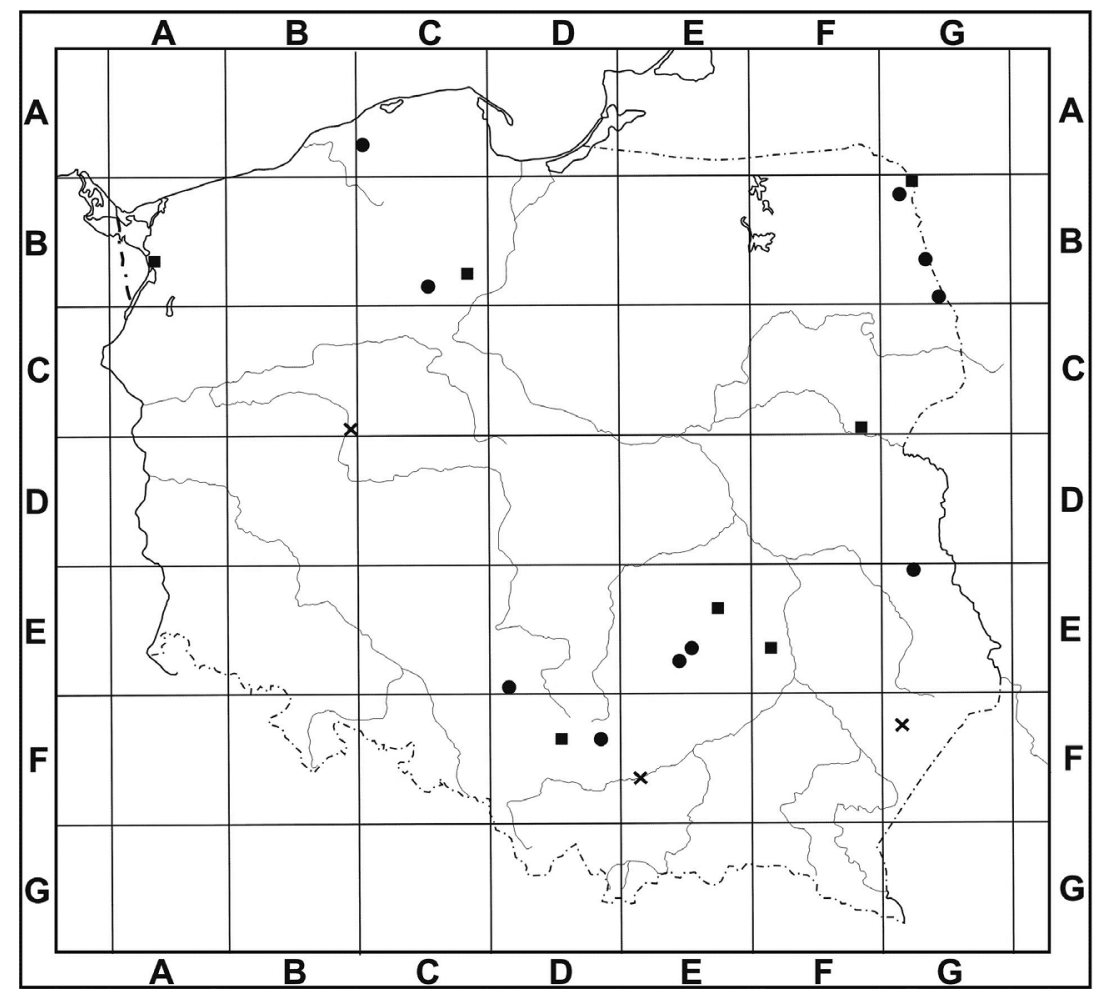

Fig. 11. Distribution map of Carex $\times$ ruedtii Kneuck.

Explanations: $\times$ - sedges collected till 1960, - - sedges collected in 1961-1990,

- sedges collected in 1991-2013 


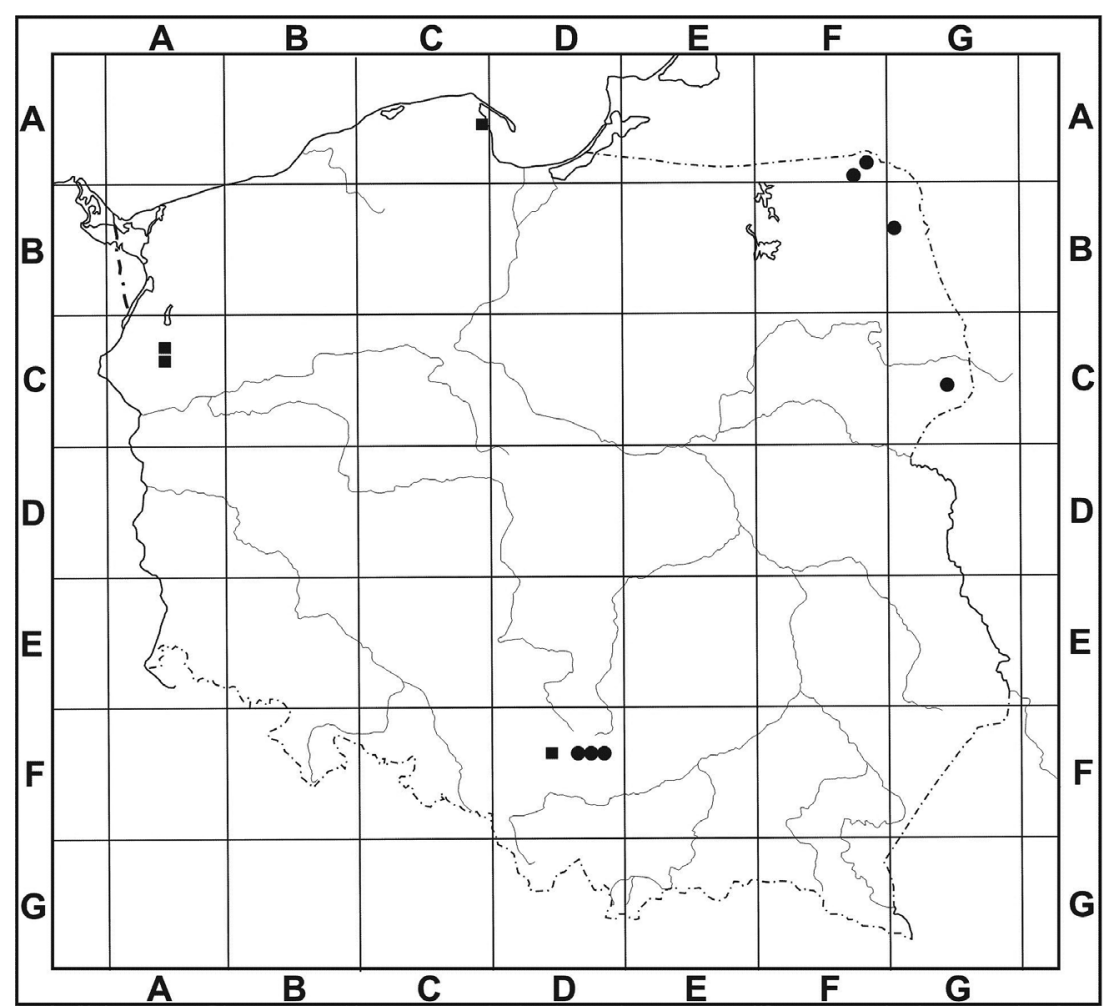

Fig. 12. Distribution map of Carex $\times$ schatzii Kneuck.

Explanations: - sedges collected in 1961-1990, $\mathbf{\square}$ - sedges collected in 1991--2013

\subsection{Carex $\times$ ruedtii Kneuck. [C. flava $\times$ C. lepido- carpa]}

Hybrid recorded mostly in northern and western Europe (Koopman 2011); also known from Canada
(Raymond 1950) and USA (Crins \& Ball 1989). In Poland found in 20 ATPOL squares in 10 provinces. Its localities are most numerous in the central and eastern part of the country (Fig. 11, Appendix 2).

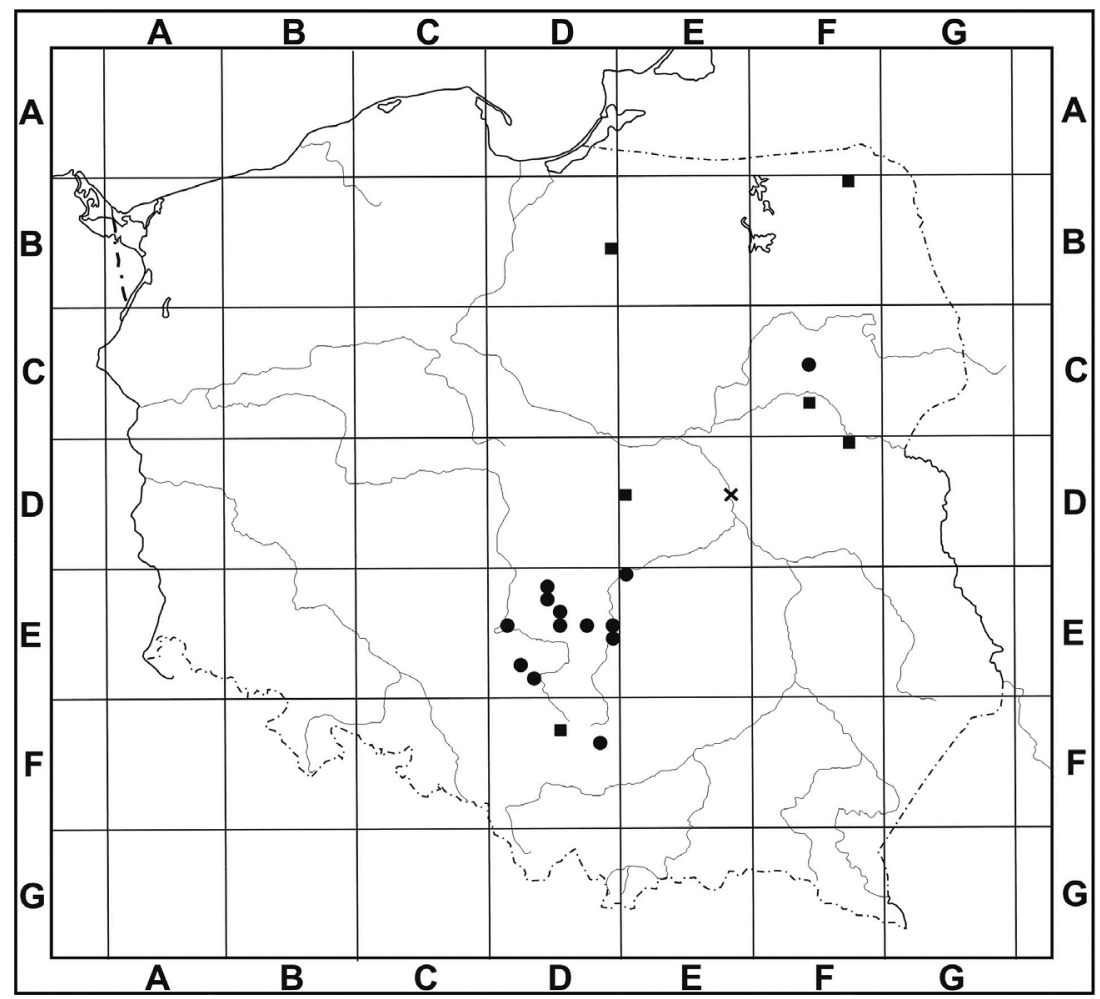

Fig. 13. Distribution map of Carex $\times$ subviridula Fernald

Explanations: $\times$ - sedges collected till 1960, - sedges collected in 1961-1990,

- sedges collected in 1991-2013 


\subsection{Carex $\times$ schatzii Kneuck. [C. lepidocarpa $\times$ C. viridula]}

Distributed mostly in northern and central Europe (Koopman 2011). In Poland it is most frequent in Małopolska and the western part of Pomerania; its localities were recorded in 11 ATPOL squares in 5 provinces (Fig. 12, Appendix 2).

\subsection{Carex $\times$ subviridula Fernald [C. flava $\times$ C. viridula]}

Hybrid distributed in Scandinavia, Germany, Belgium, France, Switzerland, Austria, Romania, Lithuania, Latvia, Estonia, and Russia (Koopman 2011); reported from Canada (Raymond 1950) and USA (Crins $\&$ Ball 1989). In Poland C. ×subviridula was found only in herbarium material (LOD, OLTC, and private collections). Its localities are most numerous in the central part of the country, especially in Łódź Province (Łódzkie); in total, recorded in 20 ATPOL squares in 6 provinces (Fig. 13, Appendix 2).

\section{Final remarks and conclusions}

The presented maps of distribution of sedges of the C. flava complex are based on field data and information from labels of herbarium specimens. During analysis of material from Polish herbaria, many errors in species identification were noticed on labels of taxa of the C. flava group. Most often, hybrids were misidentified, labelled as pure species, whereas relatively rarely mistakes in identification of pure taxa were noticed: $C$. viridula vs $C$. demissa or C. lepidocarpa vs $C$. flava. Most of herbarium sheets contained plants collected in 1961-1990. Unfortunately, only few herbaria included specimens collected in the last few years. Besides, some labels provided incomplete data about the site of collection, i.e. the locality name given on the label was ambiguous (as localities with the same name are found in various parts of Poland) or no information was given about the collection site (this applies particularly to specimens collected in the late 19th and early 20th century). Because of these problems, the maps presented here can be treated as a general pattern of distribution of the studied taxa in Poland, based on an analysis of all the available data.

The results indicate that C.flava s.s. is the most common species of the section Ceratocystis in Poland, while $C$. hostiana is the rarest. C. demissa is relatively abundant in southern Poland: in the mountains and in the submontane zone, especially in Małopolska, Podkarpacie, and Silesia Provinces. Its most elevated locality (about 1290 m a.s.1.) is known from the Beskid Żywiecki Mts, near Hala Miziowa. C. lepidocarpa is most numerous in Polesie and Lublin Upland, Małopolska, and Pomerania. Revision of herbarium specimens and field research did not confirm frequent occurrence of this species in the south of Poland. C. lepidocarpa typically grows on calcareous soils, in plains or on hills. In Poland its most elevated locality was at $460 \mathrm{~m}$ a.s.1. C. viridula var. viridula is frequent in Poland, whereas $C$. viridula var. pulchella has only 2 localities in the north. $C$. viridula var. viridula is relatively frequent in northern, central, and south-eastern Poland, whereas in the south-western part its localities are scattered. The most frequent hybrid in Poland is $C$. xalsatica, while $C$. xruedtii, C. $\times$ subviridula, and $C . \times$ schatzii are rarer, and the rarest hybrid are $C$. demissa $\times C$. viridula, $C . \times f u l v a$, and $C . \times$ leutzii.

\section{References}

BALL P. W. 1990. Some aspects of the phytogeography of Carex. Can. J. Bot. 68: 1462-1472.

Ball P. W. \& RezniceK A. A. 2002. Carex L. In: P. W. Ball, A. A. RezniceK \& D. F. Murray (eds.). Flora of North America north of Mexico, vol. 23, 254-572 pp. Oxford University Press, New York.

Blackstock N. \& Ashton P. A. 2001. A re-assessment of the putative Carex flava agg. (Cyperaceae) hybrids at Malham Tarn (v.c. 64): A morphometric analysis. Watsonia 23: 505-516.

Chater A. O. 1980. Carex L. In: T. G. Tutin, V. A. Heywood, N. A. Burges, D. M. Moore, S. M. Walters \& D. A. WebB (eds.). Flora Europaea, vol. 5 Alismataceae to Orchidaceae (Monocotyledones). 290-323 pp. Cambridge University Press, Cambridge.
CRins W. J. \& Ball P. W. 1989. Taxonomy of the Carex flava complex (Cyperaceae) in North America and northern Eurasia. II. Taxonomic treatment. Can. J. Bot. 67: 1048-1065.

Davies E. W. 1953a. Notes on Carex flava and its alliens. I. A sedge new to the British Isles. Watsonia 3: 66-69.

Davies E. W. 1953b. Notes on Carex flava and its alliens. IV. Geographic distribution. Watsonia 3: 80-84.

Davies E. W. 1956. The ecology and distribution of Carex flava and its allies in the British Isles. Botaniska Notiser 109: 51-74.

Egorova T. V. 1999. The sedges (Carex L.) of Russia and adjacent states (within the limits of the former USSR). 772 pp. Missouri Botanical Garden Press, Saint-Louis. 
Escudero M., Valcárcel V., Vargas P. \& Luceño M. 2010. Bipolar disjunctions in Carex: long-distance dispersal, vicariance or parallel evolution? Flora 205: 118-127.

Good R. 1974. The Geography of the Flowering Plants. 557 pp. Longman, London.

Govaerts R., Koopman J., Simpson D., Goetghebeur P., Wilson K., Egorova T. \& Bruhl J. 2010. World checklist of Cyperaceae. Kew: The Board of Trustees of the Royal Botanic Gardens. Available at http://apps. kew.org/wcsp/monocots/ (accessed 25 May 2011).

HavlícKová J. 1983. Carex flava-complex in the Czech lands. II. Notes on the geographical distribution. Preslia 55: 245-263.

Hultén E. \& Fries M. 1986a. Atlas of North European vascular plants north of the Tropic of Cancer. 3: 969-1172. Koeltz Scientific Books, Königstein.

Hultén E. \& Fries M. 1986b. Atlas of North European vascular plants north of the Tropic of Cancer. 1. pp. xvii +498 . Koeltz Scientific Books, Königstein.

JASIEwicz A. 1965. Rośliny naczyniowe Bieszczadów Zachodnich. Monogr. Bot.. 20: 3-337.

Kern J. H. \& Reichelt T. J. 1954. Carex L. In: T. Weevers, B. H. DANSER \& J. Heimans (eds.). Flora Neerlandica 1(3): 1-133. Koninklijke Nederlandse Botanische Vereniging, Amsterdam.

Kiffe K. 2001. Die Hybriden zwischen Carex hostiana und den Arten der Carex flava-Gruppe in NordrheinWestfalen. Flor. Rundbr. 35(1/2): 61-71.

Koopman J. 2011. Carex Europaea. The genus Carex L. (Cyperaceae) in Europe, 1. Accepted names, hybrids, synonyms, distribution, chromosome numbers. 726 pp. Margraf Publishers, Weikersheim.

Kuchel S. D. \& Bruederle L. P. 2000. Allozyme data support a eurasian origin for Carex viridula subsp. viridula var. viridula (Cyperaceae). Madroño 3: 147-158.

KuKKonen I. 1984. New infraspecific taxa and nomenclatural combinations in Carex (Cyperaceae) in the Flora Iranica area. Ann. Bot. Fen. 21: 383-389.

Meusel H., JÄGer E. \& Weinert E. 1965. Vergleichende Chorologie der zentraleuropäischen Flora. I. Text 583 pp., Karten 258 pp. Gustav Fischer Verlag, Jena.

Mirek Z., Musią Ł. \& Wójcicki J. J. 1997. Polish Herbaria. Polish Bot. Studies Guidebook Series 8: 1-116.

Moore D. M. \& Chater A. O. 1971. Studies of bipolar disjunct species. 1. Carex. Botaniska Notiser 124: 317-334.
PYKÄLÄ J. 1994. The ecology and distribution of Carex lepidocarpa subsp. lepidocarpa in Finland. Ann. Bot. Fen. 31: 261-274.

PykäLÄ J. \& Toivonen H. 1994. Taxonomy of the Carex flava complex (Cyperaceae) in Finland. Nordic J. Bot. 14: 173-191.

RAYmond P. M. 1950. Les Cypéracées de l'île anticosti: Carex et Kobresia. Canadian Journal of Research C, 28: 406-444.

RAYMOND P. M. 1951. Sedges as material for phytogeographical studies. Mémoires du Jardin Botanique Montréal 20: 1-23.

Schmid B. 1981. Die Verbreitung der Artengruppe Carex flava L. s.1. in der Schweiz. Botanica Helvetica 91: 3-8.

Schultze-Motel W. 1980. Carex L. In: Gustav Hegi. Illustrierte Flora von Mitteleuropa. 2(1): 96-274. Verlag Paul Parey, Berlin-Hamburg.

SoтEK Z. 2010. Distribution patterns, history, and dynamics of peatland vascular plants in Pomerania (NW Poland). Biodiv. Res. Conserv. 18: 1-82.

Stoeva M. P. \& ŠTePÁnKovÁ J. 1990. Variation patterns within the Carex flava agg. in Bulgaria and Czechoslovakia. Preslia 62: 1-24.

WIEcCŁAW H. 2014. Carex flava aggregate (section Ceratocystis, Cyperaceae) in Poland: taxonomy, morphological variation, and soil conditions. Biodiv. Res. Conserv. 33: 3-47.

WiĘCŁAW H. \& KoOpMAn J. 2013. Numerical analysis of morphology of natural hybrids between Carex hostiana DC. and the members of Carex flava agg. (Cyperaceae). Nordic J. Bot. 31(4): 464-472.

Villaverde T., Martín-Bravo S., Escudero M. \& Luceño M. 2012. Extreme phylogeography in Carex (Cyperaceae). Informatore Botanico Italiano 44: 58-6.

ZAJĄC A. 1968. Carex serotina Mér. subsp. pulchella (Lönnr.) v. Ooststr. w Polsce. Fragm. Flor. Geobot. 14(2): 205-211.

ZAJĄC A. 1978. Atlas of distribution of vascular plants in Poland. Taxon 27(5-6): 481-484.

ZAJAcC A. \& ZAJĄC M. (eds.). 2001. Distribution Atlas of Vascular Plants in Poland. xii+714 pp. Edited by Laboratory of Computer Chorology, Institute of Botany, Jagiellonian University, Cracow.

Zając M. \& ZAJĄC A. 2009. The geographical elements of native flora of Poland. $94 \mathrm{pp}$. Edited by Laboratory of Computer Chorology, Institute of Botany, Jagiellonian University, Kraków. 
Appendix 1. Locality lists of Carex taxa within the section Ceratocystis

\section{Carex demissa Hornem.}

Dolnośląskie. AE29: S of Gozdów, meadow, leg. E. Kuźniewski 1967 (SZUB); S of Bolesławiec, meadow, leg. Suszyńska 1969 (WRSL). AE56: Leśna, river alluvia, leg. Powiertowski 1965 (LOD). AE78: Karpniki, near green tourist trail along a stream, leg. H. Więcław 2011 (**). AE89: bottom of glacial kettle Mały Staw, leg. A. Sokołowski 1955 (BIL); Karpacz, roadside near green tourist trail, between Polana Bronka Czecha (glade) and Rozdroże Łomnickie, leg. H. Więcław $2011(* *)$. BE26: Mojęcice-Krzydlina Mała, meadow, leg. A. Krawiecowa 1964 (WRSL). BE60: Jelenia Góra, moist depression in forest between Jeżów and Dziwiszów, leg. A. Sokołowski 1975 (BIL). BE70: Mysłakowice, roadside ditch leg. H. Więcław 2011 $(* *)$. BE71: Karpniki, forest roadside near blue tourist trail leg. H. Więcław $2011(* *)$; Karpniki, moist forest roadside near yellow tourist trail, leg. H. Więcław 2011 (**). BE77: Sulistrowiczki, meadow, leg. M. Ciaciura 1988 (SZUB). BE84: Jedlina Zdrój, moist meadow, leg. W. Włoch 1970 (WRSL). BF23: Darnków, moist meadow, leg. Z. Gołąb 1969 (WRSL). BF47: Międzygórze, pasture near farmstay "Rancho u Bolka", leg. H. Więcław 2011 (**); Międzygórze, forest roadside near yellow tourist trail, leg. H. Więcław $2011(* *)$.

Kujawsko-pomorskie. CB75: Bladowo, leg. E. F. Klinsmann, no date (TRN). DC10: Skąpe, meadow, leg. K. Kępczyński 1960 (TRN). DC56: Modrzewie, meadow, leg. K. Kępczyński 1960 (TRN). DC63: Krzyżówki, leg. K. Kępczyński 1961 (TRN). DC84: Wójtowskie Lake, leg. M. Gośniak 1964 (TRN).

Lubelskie. FD42: Rudnik, transition mire, leg. D. Fijałkowski 1964 (LBLM). FD45: Ławki, fen, leg. Z. Głowacki 2000 (WSRP). FE38: Wilczopole, meadow, leg. D. Fijałkowski 1965 (LBLM). FE98: Frampol, Błoto Rakowskie, transition mire, leg. T. Krzaczek 1959 (LBLM). FF19: Majdan Nowy, field, leg. unknown, no date (SZUB). FF27: Lipiny Dolne, near a ditch in farmland, leg. A. Michalewska 2003 (KRA). FF28: Potok Górny, moist drainage ditch, leg. A. Michalewska 2003 (KRA). GE01: Załucze Stare, Lake Łukie, transition mire, leg. D. Fijałkowski 1951 (LBLM). GE02: Wytyczno near Włodawa, Lake Wytyckie, mire, leg. D. Fijałkowski 1951 (LBLM). GE11: Kaniwola, Lake Nadrybie, transition mire, leg. D. Fijałkowski 1959 (LBLM). GE13: Tarnów near Urszulin, meadow, leg. D. Fijałkowski 1965 (LBLM). GE44: Strachosław, meadow, leg. D. Fijałkowski 1963 (LBLM). GF01: Górecko Kościelne, Szum river valley, leg. D. Fijałkowski 1965 (LBLM). GF12: Oseredek, mire, leg. T. Krzaczek 1960 (LBLM). GF13: Kocudza, fir-pine forest, leg. D. Fijałkowski 1950 (LBLM). GF22: Rebizanty, meadows near Tanew river, leg. D. Fijałkowski 1965 (LBLM).

Lubuskie. AC47: Lośno, wet meadow, leg. M. Myśliwy 1999 (SZUB). AD95: NE of Witoszyn Górny, wet meadow, leg. M. Ciaciura 1962 (SZUB). AD96: Jankowa Żagańska, under power lines, leg. P. Kobierski $2011\left(^{*}\right)$.

Lódzkie. DE37: Wilkoszewice, meadow, leg. L. Fagasiewicz 1977 (LOD). ED51: Ruda-Chlebacz, alluvial alder forest, leg. M. Karolak 1980 (LOD).

Małopolskie. DF55: Chrzanów-Kościelec, muddy depression on a pasture near Chechło Reservoir (= Lake Chechelskie), leg. L. Olesiński 1972 (KRAM). DF65: Libiąż, wet pine-birch forest near a railway embankment, leg. I. Mazaraki 1968 (KRAM). DF69: Królówki near Kostrza, ditch, leg. K. Ciońćka 1993 (KRA). DF79: Kraków, Borek Fałęcki, leg. W. Kulczyński 1874 (KRAM); Kraków, Borek Fałęcki, mire, leg. T. Tacik 1951 (KRAM). DF85: Wieprz near Andrychów, wet meadow, leg. M. Łańcucka 1935 (KRAM). DF87: Bugaj, near "Dróżki Pana Jezusa” (Jesus' Trails), leg. K. Jędrzejko 1999 (KRAM). DF96: Śleszowice, waterlogged field road, leg. B. Kotońska 1986 (KRA). DF97: Budzów, wet meadow, leg. D. Kempa 2006 (KRA); Skawce, moist meadow, leg. W. Maczek 1967 (KRAM). DF98: nature reserve "Las Gościbia", Gościbka stream valley, 460 m a.s.l., leg. H. \& T. Tacik 1965 (KRAM); Jaworze, Gościbka stream valley, fen in groundwater seepage area, 520 m a.s.l, leg. H. \& T. Tacik 1964 (KRAM); Podlesie, fen in groundwater seepage area, 440 m a.s.l., leg. H. \& T. Tacik 1965 (KRAM); Kijówka, Harbutowicki stream valley, fen in groundwater seepage area, 400 m a.s.1., leg. H. \& T. Tacik 1964 (KRAM). DF99: Więciórka, peaty meadow, leg. I. Błaszczyk 1967 (KRA); Stróża, Chaciabówka Mount, near a ditch with water along a road, 410 m a.s.l., leg. W. Bartoszek 1996 (KRA). DG06: Żurawnica, meadow, leg. B. Kotońska 1986 (KRA). DG08: Wieprzec-Skomielna, Magurka Mount, southern slope, waterhole, leg. W. Bartoszek 1997 (KRA). DG09: Kokorzyk Mount (= Fuck's Mount), above Skomielna Czarna, wetland near green tourist trail, $560 \mathrm{~m}$ a.s.l., leg. W. Bartoszek 1997 (KRA); Tokarnia, escarpment near Więciórka stream, 420 m a.s.l., leg. W. Bartoszek 1997 (KRA). DG18: SSE of Sidzina Górna, wet meadow, 765 m a.s.l., leg. M. \& J. Guzikowie 1964 (KRAM). DG27: Łopień, between streams Rybny Potok and Suchy Potok, fen in groundwater seepage area, 190 m a.s.1., leg. K. Towpasz 1968 (KRA); Lipnica Wielka, Cyrak Mount, SE part, meadow, 780 m a.s.l., leg. M. Guzikowa 1963 (KRAM); Lipnica Mała, partly flooded meadow at bottom of Sykla stream valley, $750 \mathrm{~m}$ a.s.l., leg. M. \& J. Guzikowie 1966 (KRAM), NE of Kiczorka, peaty meadow, 840 m a.s.l, leg. B. \& L. Stuchlikowie 1956 (KRAM). DG28: Podszkle, Bukowiński stream valley, wet glade in spruce forest, 815 m a.s.l., leg. M. \& J. Guzikowie 1965 (KRAM); Podszkle, wet meadow, leg. M. \& J. Guzikowie 1965 (KRAM); Podwilk, waterlogged depression in a meadow behind church, 690 m a.s.l., leg. M. \& J. Guzikowie 1966 (KRAM); Podwilk, NE part, pasture near major road, 685 m a.s.l., leg. M. \& J. Guzikowie 1966 (KRAM); E of Podszkle, Pająków Mount, SE slope, wet depression in a meadow, 865 m a.s.l., leg. M. \& J. Guzikowie 1965 (KRAM); Podszkle, stream valley ESE of church, wet meadow, leg. M. \& J. Guzikowie 1965 (KRAM); Podszkle, stream valley, W of church, muddy places, 680 m a.s.l., leg. M. \& J. Guzikowie 1965 (KRAM); between 
Załuczne and Piekielnik, meadow, 680 m a.s.l., leg. M. \& J. Guzikowie 1962 (KRAM); N of Podwilk, spruce forest edge, on slopes of Bębeński stream valley, 685 m a.s.l., leg. M. \& J. Guzikowie 1966 (KRAM); N of Harkabuz, mountain pass SE of Łysa Góra, moist meadow, 810 m a.s.l., leg. M. \& J. Guzikowie 1966 (KRAM); between Podszkle and Bukowina, flooded clay pit, partly overgrown with emergent vegetation, 850 m a.s.l., leg. M. \& J. Guzikowie 1965 (KRAM). DG59: mountain pass Przełęcz na Patykach (now known as Przełęcz Białego) leg. I. Król 1914 (KRA); Kościelisko, near sanatorium Salamandra (S slope of Butorów), fen in groundwater seepage area, leg. Z. Mirek 1985 (ZAMU); Kościelisko, above Salamandra, eutrophic fen in groundwater seepage area, $1060 \mathrm{~m}$ a.s.l., leg. Z. Mirek 1985 (ZAMU); Dolina Strążyska (valley), leg. unknown 1906 (KRAM); glade near road to Stoły, 1240 m a.s.l., leg. B. Chwastowski 1977 (KRAM); Dolina za Bramką (valley), leg. unknown 1881 (KRAM); Zakopane, wet meadow, leg. K. Chronowska 1954 (KRAM). EF61: Niepołomice Forest, W part, leg. L. Nowak 1987 (KRAM). EF72: N of Grodkowice, Niepołomice Forest, mud, leg. M. Zającówna 1953 (KRAM). EF77: Skrzyszów, wet meadow, leg. K. Towpasz 1994 (KRA). EF90: Lipnik, moist meadow, leg. W. Bartoszek 1994 (KRA); Trzemeśnia Brzeg, wet meadow, 350 m a.s.l., leg. K. Stawowczyk 2004 (KRA); Bulina near Osieczany, meadow, 380 m a.s.1., leg. M. Ralska 1956 (KRAM); Pcim, leg. K. Starmach 1925 (KRAM). EF93: Żmiąca, meadow, leg. M. Szewczyk 1978 (KRAM). EG01: Kasina Wielka, wet meadow, 560 m a.s.l., leg. K. Towpasz 1968 (KRA). EG02: Chyszówki, wet meadow, $640 \mathrm{~m}$ a.s.l., leg. K. Towpasz 1968 (KRA); Hajdowska Góra (Słopnice), wet meadow, 560 m a.s.l., leg. K. Towpasz 1968 (KRA). EG03: Pisarzowa, ditch, leg. J. Staszkiewicz 1964 (KRAM); Sowliny near Limanowa, fen in groundwater seepage area in alder wood, leg. M. Szewczyk 1979 (KRAM); N slope of Łysa Góra, moist meadow, leg. M. Szewczyk 1979 (KRAM). EG11: mountain pass between Półrzeczka and Wilczyce, wet old field, $740 \mathrm{~m}$ a.s.l., leg. K. Towpasz 1970 (KRA). EG12: Mogilnica Mount, Czarny stream valley, fen in groundwater seepage area, $760 \mathrm{~m}$ a.s.l., leg. K. Towpasz 1969 (KRA). EG17: Kąclowa near old church, peatland, leg. D. Wróbel 2004 (KRAM). EG20: between Lasek and Klikuszowa, damp depression in a pasture, 610 m a.s.l., leg. M. \& J. Guzikowie 1966 (KRAM); Gorce Mts, Turbacz, wetlands near road, 1245 m a.s.l., leg. J. Kornaś 1955 (KRAM). EG23: Pasmo Radziejowej (mountain range), moist place on a path, leg. K. Stawowczyk 2008 (KRA). EG24: Jaworzynka stream valley, along a forest road, $610 \mathrm{~m}$ a.s.l., leg. K. Stawowczyk 2008 (KRA); Przysietnica stream valley, damp places near a wood shed, leg. K. Stawowczyk 2008 (KRA). EG28: Czarna near Uście Gorlickie, meadow, leg. K. Oklejewicz 1994 (*). EG29: Smerekowiec, wet meadow, leg. K. Oklejewicz 1994 (*); Banica, roadside, leg. K. Oklejewicz 1994 (*). EG33: Jarmuta, wet places near a quarry, leg. B. Pietras 1967 (KRA); Skotnicki Potok, stream valley, $510 \mathrm{~m}$ a.s.l., leg. K. Stawowczyk 2008 (KRA). EG34: Szczawnica, valley of Kotelniczy Potok (stream), damp roadsides, 730 m a.s.l., leg. K. Stawowczyk 2006 (KRA); Czercz stream valley, leg. B. Pawłowski 1918 (KRAM). EG35: Obłazy Ryterskie, moist place on a path, leg. K. Stawowczyk 2007 (KRA); Czercz stream valley, damp places along stream, leg. K. Stawowczyk 2007 (KRA). Mazowieckie. EC99: Kurówka, meadow, leg. Z. Głowacki 1980 (WSRP). EE23: Przysucha, Zapniów Forest, fen, leg. M. Podgórska 2006 (KRA). EE24: W of Rzuców, road in moist oak-hornbeam forest, leg. M. Nobis 2003 (KRA); Dluga Brzezina, forest road, leg. M. Podgórska 2006 (KRA). EE35: Budki III near Szydłowiec, moist roadside, leg. M. Nobis 2003 (KRA). EE45: Majdów, nature reserve "Cisowy Majdów”, peatland, leg. J. Grygiel \& E. Bróż 1976 (KTC). FC94: Kostki-Pieńki, wet forest meadow, leg. Z. Głowacki 1980 (WSRP). FD04: E of Paczuski, forest, leg. E. Ćwikliński 1987 (WSRP). FE23: Gołe Łąki, mire, leg. J. Sikora 1997 (WSRP).

Opolskie. CF06: Walidrogi, wet forest glade, leg. A. Duda 1977 (KTU). CF09: Liszczak, pond edge, leg. G. Chmiel 1977 (KTU). ). CF13: NE of Dobrzyków, meadow, leg. E. Kuźniewski 1967 (SZUB); Rzymkowice, wetland, leg. W. Głombica 1978 (KTU). CF32: Prudnik, leg. Barthel 1884 (TRN).

Podkarpackie. FF16: SE of Bukowina, wet meadow, leg. A. Michalewska 2003 (KRA); Wólka Bielińska, wet meadow, leg. A. Michalewska 2003 (KRA); SW of Dąbrowica, wet forest road, leg. A. Michalewska 2004 (KRA); 750 m SW of Dąbrowica, wet forest roadside, leg. A. Michalewska 2004 (KRA). FF17: S of E part of village Harasiuki, near a watercourse, leg. A. Michalewska 2004 (KRA). FF28: 2 km N of Luchów Dolny, SW of Jastrzębiec, moist meadow near forest, leg. A. Michalewska 2004 (KRA); E of village buildings of Brzyska Wola, moist old field near forest, leg. A. Michalewska 2004 (KRA). FF38: E of Dą̧rowica Duża, meadow near partly overgrown waterhole, leg. A. Michalewska 2003 (KRA); E of Dąbrowica Duża, meadow near road Słoboda-Kolonia Polska, leg. A. Michalewska 2003 (KRA); Brzyska Wola, moist ditch, leg. A. Michalewska 2003 (KRA); ); Brzyska Wola, wet meadow, leg. A. Michalewska 2003 (KRA); Słoboda Duża-Kolonia Polska, waterlogged site near road, leg. A. Nobis 2005 (KRA). FF50: Nagoszyn, wet pasture, leg. C. Brzostowska 1977 (KRA); Wola Nagoszyńska, fen, leg. C. Brzostowska 1977 (KRA). FF80: Wola Brzostecka, emergent vegetation, leg. K. Towpasz 1981 (KRA). FG01: Łężyny near Jasło, edge of a waterhole, leg. K. Oklejewicz 1989 (KRA). FG03: between Widacz and Haczów, fen, leg. K. Oklejewicz 1990 (KRA). FG21: Ropianka, waterlogged site in fir-beech forest, leg. K. Oklejewicz $1999(*)$; settlement Olchowiec, E part, fen in groundwater seepage area, leg. J. Mitka 1999 (KRA); Ropianka, wet glade in beech forest, leg. K. Oklejewicz 2004 (OLTC). FG22: Cergowa Mount near Dukla, wet road in beech forest, leg. K. Oklejewicz $1994\left(^{*}\right.$ ); Lipowica, clayey site near a bus stop, leg. K. Oklejewicz 2000 (*). FG23: Zawadka Rymanowska, E part, edge of fish pond,

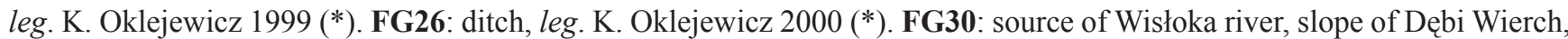
alder wood, leg. M. Zarzyka 1999 (KRA). FG34: Góra 727, Low Beskids (Beskid Niski Mts), drying site where wood was stored, leg. K. Oklejewicz 2000 (*); Berdo Mount, waterlogged, rocky bank of Wisłoka river, leg. K. Oklejewicz 2000 (*); Jasiel, forest road, leg. K. Oklejewicz 2007 (KRA). FG46: Bystre near Baligród, fen in groundwater seepage area, $500 \mathrm{~m}$ a.s.l., leg. A. Jasiewicz 1962 (KRAM). GG70: Wołosate, forest roadside in direction of Szczawinka river leg. H. Więcław 
2010 (**); Wołosate, meadow in Szczawinka river valley, leg. H. Więcław 2010 (**); Wołosate, Przełęcz Beskid (mountain pass), roadside ditch, leg. H. Więcław $2010(* *)$.

Podlaskie. FA98: Udryn, meadow, leg. A. Kawecka 1980 (BIL). FB69: Białko, Kępina mire, leg. Z. Głowacki 1974 (WSRP). FC05: Jedwabne, wet meadow, leg. A. Sokołowski 1968 (BIL). GB00: Pawłówka, peaty meadow, leg. S. Fieńko 1969 (BIL). GB11: Giby, peatland, near Lake Dumbel, leg. H. Werblan-Jakubiec 1975 (WA). GB41: Lipsk, meadow, leg. J. Żurawski 1974 (BIL).

Pomorskie. BB29: Miastko, Lake Lednik, mire, leg. J. Nowak 1978 (SLTC). CA38: Jastrzębia Góra, fen in groundwater seepage area, leg. H. Piękoś 1964 (KRAM). CA49: Wielka Wieś near Władysławowo, moist pasture near Bay of Puck, leg. H. \& T. Tacik 1962 (KRAM). CA50: Retowo, moist meadow S of Lake Gardno, leg. H. Więcław 2011 (**). CA51: N of Gardna Wielka, pasture, leg. H. Więcław 2011 (**). CA52: S of Kluki, moist meadow, leg. H. Więcław 2011 (**). CA59: W of Rewa, Reda river valley, meadow, leg. M. Jańczak 1971 (UGDA). CA89: Oliwa Forest (Lasy Oliwskie), leg. A. Wangerin 1925 (TRN). CB16: Sarnowy, forest mire near Lake Drzędno, leg. E. Jasińska 1974 (UGDA). CB56: Konewka near Czersk, patch of alluvial forest near village, leg. F. Borys 1964 (POZ).

Śląskie. CF67: Obora, wet glade leg. I. Żurek 1963 (WRSL). CF79: Krostoszowice, moist edge of a pond, N of mine shaft, leg. K. Prudel 1987 (KTU). DE72: Rybno, peaty wetlands, leg. J. Śledziona 1987 (KTU). DE82: Blachownia, mire, leg. B. Gajos-Kędzierska 1959 (KRA); Gać near Blachownia, meadow, leg. J. Hereźniak 1972 (LOD). DE83: Gliny-Wygoda near Dźbów, wetlands, mining subsidence pools, leg. J. Hereźniak 1972 (LOD). DE93: Kijas near Konopiska, meadow, leg. J. Hereźniak 1972 (LOD); Konopiska, bottom of drying pond, leg. J. Hereźniak 1972 (LOD). ). DF11: Tłuczykąt, moist medow, leg. E. Głogowska 2004 (KTU). DF23: Psary, moist medow, leg. B. Opiełka 1999 (KTU). DF25: Tucznawa, moist medow, leg. K. Jędrzejko 1974 (KTU). DF32: Bytom Szombierki, meadow, leg. G. Kloula 1977 (KTU). DF33: Będzin, Łagisz power station on Psarka river, fens in groundwater seepage areas among moist meadows, leg. A. Błońska $2011(*)$. DF34: Będzin Bory, fen in groundwater seepage area in Czarna Przemsza river valley, leg. A. Sendek 1976 (KTU); meadow near a pond W of Łagisz power station, leg. K. Jędrzejko, H. Klama \& J. Żarnowiec 1981 (KRA); Dąbrowa Górnicza Pogoria, peatland near a lake, leg. A. Sendek 1983 (KTU). DF35: N of Łąka, forest road, leg. B. Babczyńska-Sendek 1988 (*). DF42: Huta Katowice, Pogoria, leg. K. Rostański 1975 (KTU). DF43: Katowice Muchowiec, old airport, leg. A. Sendek 1975 (KTU); Katowice Muchowiec, fen in groundwater seepage area, leg. A. Sendek 1976 (KTU). DF45: Cieśle, Sosnowiec Maczki, sedge bed, leg. A. Sendek 1976 (KTU); Cieśle, meadow, leg. A. Sendek 1976 (KRA). DF51: Łaziska, Górne Kąty, wet meadow, leg. A. Urbisz 1995 (KTU); Zawiść-Pasieki, alluvial alder forest, leg. A. Balon 1983 (KTU). DF52: Mikołów, Dolina Jamny (stream valley), wet meadow, leg. M. Dobrzańska 1977 (KTU). DF60: Żory near Lake Śmieszek, leg. J. Gałuszka 1931 (KTU). DF82: Zabrzeg, wet meadow, leg. B. Bolek 1988 (KTU). DF94: Kosarzyska, 510 m a.s.1., leg. B. Kotońska 1980 (KRA); Żarnówka Mała, meadow in river valley, leg. I. Wojtyra 1980 (KTU); Kozy, 550 m a.s.1., leg. B. Kotońska 1986 (KRA). DG02: Szczyrk, moist meadow under slope of Beskidek Mount leg. W. Ganszer 1960 (SZUB); Brenna, spruce forest, leg. M. Broda 1967 (KRA); Brenna, wet meadow, leg. S. Cabała 1974 (KTU); Brenna, Leśnica river head, above ponds, leg. K. Rostański 1974 (KTU). DG03: Szczyrk, Polana Hondraska (glade), slope above road, leg. K. Rostański 1987 (KTU). DG04: Oczków, peaty patch, leg. B. Kotońska 1986 (KRA). DG05: Kocoń, wet road, leg. B. Kotońska 1985 (KRA). DG12: W slope of Malinowska Skała, fen in groundwater seepage area, 1040 m a.s.1., leg. K. Zarzycki 1956 (KRAM). DG14: Juszczyna, in Juszczynka stream valley, 520 m a.s.l., leg. K. Białecka 1960 (KRA); Potok Pawlusianki (stream), wetland near road, 390 m a.s.1., leg. K. Nowak 1986 (KRAM). DG22: Olecki Górne, wet meadow, leg. M. Dudzik 1972 (LOD); Istebna, peatland near forest, leg. A. Sendek 1978 (KTU). DG23: Kamesznica-Złotna, moist roadside, leg. D. Papiernik \& B. Babczyńska-Sendek 1987 (*); Nieledwia, wet meadow, leg. I. Hola 1999 (KTU); Szare, roadside, leg. I. Hola 1999 (KTU). DG25: Pilsko, Hala Miziowa (glade), fen in groundwater seepage area, 1290 m a.s.l., leg. K. Białecka 1970 (KRA).

Świętokrzyskie. EE32: Kaczywół, wet meadow, leg. Trojecka-Brzezińska 2010 (KRA). EE34: Motyki Mount between Słoby and Końskie, peaty edge of forest, leg. M. Podgórska 2004 (KRA); Górka, transition mire, leg. M. Podgórska 2006 (KRA). EE44: Odrowąż, moist roadside in pine forest, leg. M. Podgórska 2006 (KRA). EE45: Bliżyn, fen near Bliżyński Reservoir, leg. M. Nobis 2003 (KRA); Sękorki, mossy spruce-fir forest, leg. M. Podgórska 2006 (KRA); Bliżyn, wet meadow, leg. H. Piękoś 1969 (KRAM). EE47: Starachowice, forest mire, leg. M. Nobis 2002 (KRA); N part of Mirzec village, fen edges, leg. M. Nobis 2002 (KRA). EE57: S of Bugaj district of Starachowice, moist roadside, leg. M. Nobis 2004 (KRA). EE64: Góra Wierzejska (mount), forest road, leg. E. Bróż \& Sapielak 1987 (KTC); Kielce, meadow on Silnica river, leg. B. Maciejczak 1998 (KTC); Miedziana Góra (mount), thicket, leg. I. Kulej \& B. Maciejczak 1986 (KTC); fen in groundwater seepage area on Lubrzanka river drainage divide, leg. E. Bróż \& D. Strzelecka 1987 (KTC). EE74: Kielce, pine forest behind shooting range, leg. K. Kaznowski 1927 (KRAM); Kielce, meadow, leg. U. Misztal \& B. Maciejczak 1987 (KTC); Kielce, wet meadow near allotments (gardens), leg. A. Ławik \& B. Maciejczak 1987 (KTC); NE of Suków-Borki, mire, leg. G. Łazarski 2013 (KRA); Kielce Podlesie, E of Ściegiennego Street, wetlands near roadside ditch, leg. G. Łazarski 2013 (KRA); Kielce Baranówek, N slope of Telegraf Mount, moist roadside, leg. G. Lazarski (KRA). EE84: Piaseczna Górka, NE of Bilcza, wet meadow, leg. G. Łazarski 2013 (KRA). EE86: Widełki, wetland, leg. W. Paul 1988 (KRAM). EF08: Wola Wiśniowska, peatland near forest, leg. unknown 1973 (KRA). EF36: Ostrowce near Nowy Korczyn, wet meadow, leg. B. Chwastowski 1978 (KRAM). FE70: Orłowiny, leg. W. Paul 1990 (KRAM).

Warmińsko-Mazurskie. DB76: Iława, E edge of Lake Karaś, sedge bed, leg. H. Wojciechowska \& B. Polakowski 1954 (OLTC). EB14: Kiersnowo, meadow on drained peatland, leg. L. Olesiński 1968 (OLTC). EB24: Pierwagi, wet meadow, leg. 
L. Olesiński 1968 (OLTC). EB56: Targowska Wólka, meadow, leg. T. Szarejko 1971 (OLTC). EB64: Michałki, bottom of former fish pond, leg. M. Środa 2002 (OLTC).

Wielkopolskie. BC37: Jabłonowo Wlkopolskie., wet meadow, leg. M. Anczyńska 1980 (POZ).

Zachodniopomorskie. AB22: Lubiewo, meadows near forester's lodge, leg. H. Piotrowska 1958 (UGDA). AB24: N of Kołczewo, mossy meadows, leg. H. Piotrowska 1958 (UGDA). AB29: Jarkowo near Kołobrzeg, springhead, leg. M. Jasnowski 1964, 1965 (SZCZ). AC18: Sądów, mire, leg. M. Jasnowski 1966 (SZCZ). AC29: S of Żeńsko, about 8 km S of Choszczno, meadow, leg. J. Koopman 2010 (*). AC47: Lubociesz, meadow, leg. M. Myśliwy 1999 (SZUB). BA91: Bagicz, meadow and roadside depression, N of road Sianożęty-Bagicz, leg. H. Więcław 2011 (**). BB66: Szczecinek, S edge of Lake Śniadowo, leg. I. Lebiecka 1993 (SLTC). BC00: Kiełpino near Choszczno, forest meadow, leg. H. Więcław 2011 (**).

\section{Carex flava $\mathbf{L}$.}

Dolnośląskie. AE26: N of Węgliniec, peatland near railway tracks, leg. Z. Dajdok, J. Zalewska-Gałosz \& A. Nowak 2006 (*). AE57: W of Złotniki Lubańskie, forest clearing, leg. E. Panek 1971 (WRSL). AE78: Karpniki, green tourist trail along a stream, leg. H. Więcław 2011 (**). AE79: Cicha Dolina (valley), Karkonosze Mts, wet meadows and peatlands, leg. Z. Wiącek 1965 (WRSL). BD72: Grochowice, ditch in forest, leg. K. Baraniecka 1980 (WRSL). BD95: Uszczonów, wetland, leg. A. Fortuniak 1978 (LOD). BE15: Dziewin, wet meadow in Odra river valley, leg. E. Kozioł 1997 (WRSL). BE16: N of Wrzosy, wet meadow, leg. A. Krawiecowa 1964 (WRSL). BE21: Chojnów, wet ditch, leg. Z. Ruchlewicz 1970 (WRSL). BE23: Dobrzejów, meadow, leg. J. Anioł 1965 (WRSL). BE24: Rybin, meadow, leg. G. Staszewska 1967 (WRSL). BE26: Uskorz Wielki, wet meadow on Juszka river, leg. Z. Głowacki 1958 (WRSL). BE27: SE of Radecz, mire, leg. Z. Głowacki 1973 (WSRP). BE28: Oborniki Śląskie, meadow, leg. S. Godzik 1953 (KRAM, LOD, OLTC, WRSL, POZ, KTU, TRN, WA). BE36: Przedmoście, fen, leg. W. Stojanowska 1965 (WRSL). BE61: Wojcieszów Górny, alluvial forest and meadow, leg. A. Krawiecowa 1960 (WRSL); Kaczorów, wet meadow, leg. Czyżewska 1960 (WRSL); Wojcieszów Górny, meadow, leg. J. Sarosiek 1961 (WRSL). BE71: Rędziny, meadow, leg. H. Więcław 2011 (**); Karpniki, forest roadside near yellow tourist trail, leg. H. Więcław 2011 (**). BE77: W of Będkowice, moist ditch in mixed deciduous forest near road on E slopes of Ślęża, leg. W. Ganszer 1956 (KRAM); Sulistrowice, moist sites and near stream, leg. unknown 1959 (WRSL). BE81: Lubawka, wet meadow, leg. A. Dwulat 1989 (WRSL). BE82: E of Krzeszów, meadow at forest edge, leg. E. Kuźniewski 1964 (SZUB); Grzędy Górne, pasture, leg. D. Cisek 1980 (WRSL). BE84: Wałbrzych, moist meadow, leg. Rybałtowska 1970 (WRSL). BE92: N of Olszyny, meadow, leg. E. Kuźniewski 1966 (KRAM). BF23: Jerzykowice, thicket near stream, W part of village, leg. M. Ciaciura 1965 (SZUB); E of Witów, meadow near stream leg. E. Kuźniewski 1965 (SZUB); Darnków, wet meadow, leg. Z. Gołąb 1969 (WRSL). BF24: SW of Szczytniki, at forest edge near a meadow, leg. E. Kuźniewski 1965 (SZUB); SE of Kulin, meadow near stream, leg. M. Ciaciura 1965 (KRAM). BF27: Złoty Stok, Chwalisław depression, wet meadows, leg. E. Twaróg 1990 (WRSL). BF37: Siniak-Kierzna, meadow 520 m a.s.l., leg. Z. Szelag 1987 (KRAM); near Pogonna stream, 730 m a.s.l., leg. Z. Szelag 1987 (KRAM). BF46: Jaworek, moist meadow, leg. H. Więcław 2011 (**); Nowa Wieś, roadside, leg. H. Więcław 2011 (**). BF47: Jawor, moist meadow, 820 m a.s.l., leg. Z. Szeląg 1987 (KRAM); Międzygórze, Wilczka stream, meadow, 770 m a.s.l., leg. Z. Szelag 1987 (KRAM); Przełęcz Puchaczówka (mountain pass) closer to Sienna, leg. Z. Szelag 1987 (KRAM); Międzygórze, pasture near farmstay "Rancho u Bolka", leg. H. Więcław 2011 (**); Międzygórze, near yellow tourist trail, forest roadside, leg. H. Więcław $2011\left(^{* *}\right)$. BF48: Bielice, along stream in village centre, leg. H.

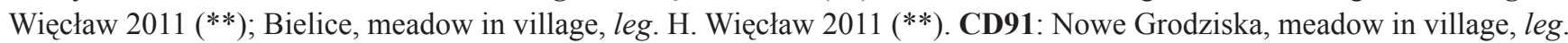
H. Więcław $2011(* *)$. CE30: E of Pruszowice, meadows among woods, leg. T. Kowal 1963 (SZUB). CE31: W of Damnik, moist meadow, leg. M. Ciaciura 1966 (KRAM).

Kujawsko-Pomorskie. CB67: SE of Zazdrość, meadow near forester's lodge, leg. H. Więcław 2009 (**); E of Laski, meadows near Grzybienica Mała river, leg. H. Więcław 2009 (**). CB68: W of Stara Rzeka, meadows near Czyściewnica river, leg. H. Więcław 2009 (**). CB73: Kamień, meadow on Kamionka, leg. K. Burzyńska 1963 (POZ). CB75: Bladowo, leg. E. F. Klinsmann (TRN); Rudzki Most, leg. M. Rejewski 1968 (TRN); Rudzki Most, wet meadow, leg. J. Błędowski 1972 (TRN). CB77: Suchom, meadow, leg. E. Krasicka-Korczyńska 1992 (BYDG). CB78: Osie, Stary Tartak (old sawmill), alder carr near edge of Lake Czerno, leg. H. Więcław 2009 (**); Wałkowiska, fen near E edge of Lake Sierosławek, leg. H. Więcław 2009 (**); Wda river valley, meadow at forest edge, leg. H. Więcław 2009 (**). CB82: Lutówko, moist meadow, leg. S. Bojakowska 1963 (POZ); Lutówko, moist meadow near road to Człuchów, leg. M. Rejewski 1964 (TRN). CB85: Piła Młyn, leg. M. Ceynowa-Giełdoń \& A. Siedlewska 1970 (TRN). CB95: Sokole-Kuźnica near Koronowo, leg. M. Ceynowa 1959 (TRN). CC15: Tryszczyn, meadow, leg. A. Szufrajda 1970 (BYDG). CC23: Trzeciewnica, moist meadow, leg. A. Godomska 1956 (LOD). CC27: Bydgoszcz Fordon, wet meadow, leg. M. Korczyński 1993 (BYDG). CC87: Przyjezierze, forest plantation, leg. W. Gugnacka 1971 (TRN); Przyjezierze, meadow on canal between Lake Czyste and Lake Ostrowskie, leg. W. Gugnacka 1971 (TRN). DC11: Węgorzyn, Czarciak mire, leg. K. Stemyska 1962 (POZ). DC17: Górzno, springhead mire, leg. M. Jasnowski 1972 (SZCZ); Gutowo-Klonowo, roadside, leg. R. Puchałka 2003 (TRN). DC31: Lubicz near Toruń, forest, leg. A. Zalewski 1889 (WA). DC35: W of Rypin, leg. A. Zalewski 1821 (WA); W of Rypin, forest pond, leg. A. Zalewski 1891 (WA). DC46: Wólka Rumunki, leg. K. Kępczyński 1960 (TRN). DC51: Ciechocinek, wet meadow, leg. R. Kobendza 2010 (WA). DC53: Maliszewo, leg. K. Kępczyński 1960 (TRN). DC64: Lipno, leg. A. Zalewski 1889 (WA); Ostrowie near Lipno, 
leg. A. Zalewski 1890 (WA). DC73: Szpetal, near Włocławek on Vistula river, leg. R. Kobendza 1913 (WA). DC83: Włocławek, Lake Widoń, leg. M. Głośniak 1964 (TRN). DC84: Lake Wójtowskie, leg. M. Gośniak 1964 (TRN). DD03: Chodecz, meadow, leg. W. Mazgalska \& L. Fagasiewicz 1949 (LOD).

Lubelskie. FD53: Kobiałki, meadow, leg. D. Fijałkowski 1964 (LBLM); Turzec, meadow, leg. D. Fijałkowski1965 (LBLM). FD55: Żdżary, fen, leg. Z. Głowacki 1982 (WSRP). FD66: Zofibór, moist forest, leg. H. Sawczuk 1977 (WSRP). FD67: Ulan, fen, leg. B. Wójcik 1976 (WSRP). FD97: Firlej, pine forest, leg. L. Rewieński 1946 (LBLM). FE03: Gołąb, wet meadow NW of railway station, leg. A. Gelo 1972 (LBLM). FE08: Serniki, fen, leg. B. Sołyga 1977 (WSRP). FE15: Markuszów, meadow, leg. Z. Buczkowska 1973 (LBLM). FE17: Nasutów, meadow, leg. H. Koporska 1918 (LBLM). FE27: Sławinek, meadow on Czechówka river, leg. L. Rewieński 1946 (LBLM). FE28: Sobianowice, meadows near Bystrzyca river, leg. D. Fijałkowski 1951 (KRA, LBLM). FE37: Zemborzyce, meadows near Bystrzyca river, leg. D. Fijałkowski 1951 (LBLM); Zemborzyce Lubelskie, meadow among hazel thickets near Bystrzyca river, leg. E. Charęzińska 1960 (LBLM); Zemborzyce Lubelskie, hay meadows in Bystrzyca river valley, leg. E. Charęzińska 1960 (LBLM). FE39: Piaski, peaty meadows, leg. St. Trzebiński 1919 (LBLM). FE88: Goraj, meadow, leg. D. Fijałkowski 1973 (LBLM). FF08: Andrzejówka, meadow, leg. D. Fijałkowski 1971 (LBLM). FF09: Brodziaki, meadow, leg. D. Fijałkowski 1971 (LBLM). FF17: Lipiny Górne-Borowina, moist sand pit near forest, leg. A. Michalewska 2003 (KRA). FF18: Wólka Biska, wet meadow, leg. J. Cios 1976 (LBLM); S of Wólka Biska, moist meadow, leg. A. Michalewska 2003 (KRA). FF19: Majdan Stary, waterlogged edge of pine forest, leg. M. Fedec 1980 (LBLM). FF27: Lipiny Dolne, near a ditch in farmland, leg. A. Michalewska 2003 (KRA); Lipiny Dolne, meadows N of village buildings, leg. A. Michalewska 2004 (KRA). FF28: S of Wola Kolońska, wet roadside, leg. A. Michalewska 2004 (KRA). FF38: Luchów Dolny, wet meadow, leg. A. Michalewska 2003 (KRA). GD81: Holendernia, meadow, leg. D. Fijałkowski 1971 (LBLM). GE01: Kolonia Wola Wereszczyńska, peatland near Lake Długie, leg. D. Fijałkowski 1964 (LBLM); Komarówka, meadow, leg. D. Fijałkowski 1964 (LBLM). GE02: Wytyczno, Krowie Bagno, mire, leg. D. Fijałkowski 1951 (KRA), leg. E. Koteja \& St. Kucharzyk 1978 (KRAB), leg. B. Chwastowski 1978 (KRAM); Wytyczno, wet meadow, leg. H. Trzcińska-Tacik 1986 (KRA). GE04: Wytyczno, polana near Durne Bagno, leg. H. Trzcińska-Tacik 1986 (KRA). GE11: Grabniak, poor fen near Lake Uśćwierz, E side, leg. J. Zalewska-Gałosz 2004 (KRA). GE13: Serniawy, alder carr, leg. J. Holub 1992 (KRAB). GE34: Chełm, calcareous meadows, leg. D. Fijałkowski 1951 (LBLM). GE35: peatland reserve "Brzeźno" near Chełm Lubelski, leg. M. Szewczyk 1978 (KRAM). GE46: Kajetanówka, stream edge, leg. D. Fijałkowski 1949 (LBLM). GE62: Krasne, meadow, leg. D. Fijałkowski 1964 (LBLM). GE81: S of Szczebrzeszyn, moist meadows near Wieprz river, leg. A. Jasiewicz 1967 (KRAM). GE86: Sahryń near Werbkowice, ditch in a meadow, leg. A. Siennicka 1997 (SZUB). GE93: Krynice near Tarnawatka, meadow, leg. D. Fijałkowski 1964 (LBLM). GF00: Bukownica, wet and mossy meadow, leg. T. Traczyk 1957 (KRAM). GF01: Górecko Kościelne, peatland in Szum river valley, leg. D. Fijałkowski 1965 (LBLM). GF02: Hutki, meadows near Wieprz river, leg. D. Fijałkowski 1962 (LBLM). GF12: Hamernia, alder woods along Sopot river, leg. D. Fijałkowski 1965 (LBLM); Oseredek, medow, leg. D. Fijałkowski 1965 (LBLM). GF21: Zamch, wet meadow in Wirowa river valley, leg. T. Krzaczek 1959 (LBLM).

Lubuskie. AC48: Santoczno, partly overgrown lake, leg. St. Skałacki 1964 (LOD); Lubociesz, wet alder forest, leg. M. Myśliwy 1999 (SZUB). AC87: Lubniewsko near Sulęcin, springhead alder carr, leg. A. Ryńska 2010 (SZCZ). AD09: Nowy Dworek, N of Lake Paklicko Wielkie, leg. W. Żukowski 1966 (POZ). BD60: Otyń, nature reserve "Bażantarnia", moist mixed deciduous forest, leg. M. Konopnicka 1979 (WRSL). BD62: Tarnów Jeziorny, wet meadow, leg. E. Kozioł 1974 (WRSL).

Lódzkie. CE39: Piaski near Wieluń, wet meadow, leg. J. Woźniak 1984 (LOD). DD44: Łęczyca, mire, leg. T. Kowal 1952 (SZUB); Błonie near Łęczyca, mire, leg. T. Kowal 1952 (SZUB). DD59: Bażantarnia, moist forest clearing, leg. A. Grzyl 1980 (LOD). DD64: Aleksandrów Łódzki, peatland near town, leg. M. Jędrzejczak no date (LOD). DD66: Łódź Łagiewniki, waterside, leg. Z. Kunitowicz 1960 (LOD). DD67: Lipka, moist meadow, leg. E. Karpik 1975 (LOD). DD68: Grzmiąca Sława, meadow, leg. M. Guldzińska 1999 (LOD). DD75: Aleksandrów Łódzki, nature reserve "Rąbień", moist meadow, leg. M. Mamiński 1980 (LOD). DD76: Wandalin, wet meadow, leg. A. Kwiecień \& B. Skrobisz 1962 (LOD). DD77: Gałkówek, moist meadow, leg. L. Leśnikowska 1973 (LOD); Wiączyń, along ditches at forest edges, leg. E. Chalearz 1978 (LOD). DD86: Bronisin, wet meadow, leg. A. Kwiecień \& B. Skrobisz 1962 (LOD). DD87: Kraszew, moist meadow, leg. E. Olszewska 1973 (LOD). DD91: Bogumiłów, moist meadow, leg. T. Załuski 1970 (LOD). DD93: Łask, meadow on Grabia river, leg. M. Tkaczuk 1957 (LOD); Okup Mały, meadow, leg. M. Tkaczuk 1957 (LOD). DD94: Mogilno, forest, leg. G. Ojrzyńska 1980 (LOD); Pałusznica, forest, leg. G. Ojrzyńska 1981 (LOD). DD95: Rydzyny, alluvial forest, leg. J. Kozemińska-Freda 1975 (LOD); S of Pabianice, meadow and ditch, leg. Z. Pawelec 1994 (KRAB). DD96: Modlica, nature reserve "Źródła Walbórki”, birch wood, leg. J. Bartel 1956 (LOD); Tuszyn, moist forest, leg. B. Cieślak \& Z. Sumińska 1973 (LOD); Modlica, moist meadow, leg. J. Barasińska 1973 (LOD). DE00: Pyszków, peaty meadow, leg. T. Załuski 1970 (LOD). DE01: Sokołów, wet pasture, leg. T. Załuski 1972 (LOD). DE10: Nowa Wieś, meadow, leg. R. Sowa 1960 (LOD); Złoczew, nature reserve "Nowa Wieś”, forest, leg. Pytlińska \& Schwarcschulc 1962 (LOD). DE13: Lubiec, pasture, leg. M. Wróblewska 1963 (LOD); Zalesie, leg. T. Załuski 1972 (TRN). DE14: Zbyszek, mire, leg. L. Kucharski \& W. Przybylińska 1977 (LOD). DE19: Zarzęcin, moist meadow, leg. J. Hereźniak, J. Staniszewska \& K. Próba 1971 (LOD). DE21: Szynkielów, peaty pool, leg. I. Góźdź 1977 (LOD). DE24: Chmielowiec, mire, leg. J. Hereźniak 1963 (LOD). DE26: Zawadów, meadow, leg. J. Jakubowska-Gabara 1984 (LOD). DE27: Piaski, alluvial forest, leg. E. Gabryszewska \& H. Marcinkowska 1978 (LOD). DE31: Siemkowice, Mokry Las, forest edge and meadow, leg. M. Pejska 1984 (LOD). DE33: Stróża, forest glade, leg. J. Jakubowska-Gabara 1981 
(LOD). DE34: Łuszczanowice, alluvial forest, leg. E. Łukasiewicz, B. Morawska \& D. Piątkowska 1978 (LOD). DE37: Trzepnica, partly overgrown pond, leg. W. Rychter 1949 (LOD); Wilkoszewice, peaty meadow, leg. L. Fagasiewicz 1977 (LOD); Bęczkowice, fen, leg. E. Kil 2009 (LOD). DE38: Piotrków, nature reserve "Wielkopole", forest glade, leg. K. Żanet 1983 (LOD). DE46: Pytowice, wet coniferous forest, leg. H. Rutowiczowa \& J. Olenderek 1977 (LOD). DE47: Kodrąb, meadow on Widawka river, leg. J. Hereźniak 1965 (LOD). DE49: E of Trupień, mire, leg. L. Fagasiewicz 1958 (LOD); Trupień on Pilica river, peaty meadow, leg. L. Fagasiewicz 1960 (LOD); Bąkowa Góra (mount), fen, leg. P. Witosławski 1981 (LOD). DE57: Kobiele Wielkie, wet meadow, leg. K. Sztampke 1958 (LOD). DE59: Przedbórz on Pilica river, leg. J. Lembke 1947 (LOD); Grobla, fen on Pilica river, leg. L. Fagasiewicz 1960 (LOD); Wierzchlas near Wojciechów, flooded depression in alder wood, leg. U. Ziętal 1971 (LOD). DE66: Jasien, forest reserve with yew, thickets, leg. E. Cajgler 1958 (LOD). DE67: Jasień, forest, leg. H. Urbanek 1959 (LOD); Żytno, forest mire, leg. H. Urbanek 1968 (LOD). DE78: Dębowiec near Silniczka, forest meadow, leg. R. Olaczek 1964 (LOD); Domaniew near Żytno, oak-hornbeam forest, leg. B. Chyrczakowska \& K. Bańkowska 1965 (LOD). DF51: Strugi near Kochlew, meadow S of Florian's Spring, leg. H. Więcław 2010 (**). ED40: Borowiny, alluvial forest, leg. M. Chabior 1997 (LOD). ED41: Ruda, right side of Rokita stream valley, meadow, leg. M. Owczarek 1996 (LOD). ED51: Ruda-Chlebacz, alluvial alder forest, leg. M. Karolak 1980 (LOD); Ruda, Bolimów Forest (Puszcza Bolimowska), peaty meadow, leg. W. Pisarek 1983 (LOD); Grabina, meadow, leg. W. Pisarek 1983 (LOD). ED92: Żądłowice reserve, mire, leg. R. Sowa 1962 (LOD). ED92: Żądłowice, alder carr, peatland, leg. J. Jakubowska, St. Stępniak \& E. Zawadzka 1967 (LOD). EE00: Tomaszów Mazowiecki, nature reserve Niebieskie Źródła, meadow, leg. J. Mowszowicz \& R. Olaczek 1960 (LOD); Sługocice, nature reserve "Jeleń", forest meadow, leg. M. Mamiński 1980 (LOD). EE01: Dęba Opoczyńska, moist meadow, leg. A. Trojecka-Brzezińska 2010 (KRA). EE12: Mroczków Gościnny, wet meadow, leg. A. Trojecka-Brzezińska 2010 (KRA); Idzikowice, moist meadow, leg. A. Trojecka-Brzezińska 2010 (KRA). EE50: Piskorzeniec, mire, leg. H. Urbanek 1967 (LOD), leg. A. Ucińska 1968 (LOD). EE60: E of Kajetanów, meadow and ditch, leg. H. Więcław $2010(* *)$.

Małopolskie. DF27: Bydlin (close to Krzywopłoty), moist meadow, leg. B. Babczyńska-Sendek 1980 (*); Bydlin-Załęże, leg. K. Jędrzejko, J. Drobnik \& K. Pawłowski 2003 (KRAM). DF36: Bolesław, meadow, in direction of Błędów Desert (Pustynia Błędowska), leg. Dobrzański 1949 (KRA); Laski, fen in Biała river valley, leg. H. Trzcińska-Tacik 1990 (KRA). DF38: E of Wolbrom, meadow, leg. B. Babczyńska-Sendek 1980 (*); Trzyciąż, forest, leg. J. Hajduła no date (KRAB); Wolbrom-Brzozówka, fen, leg. A. Sendek 1980 (KTU); Zarzecze, fen along river, leg. A. Sendek 1980 (KTU); W of Wolbrom, meadow, leg. B. Babczyńska-Sendek 1981 (*). DF56: Dulowa, meadow at forest edge, near a drainage ditch, leg. M. Mazarki 1938 (KRA); Dulowa, wet ditch near railway tracks, leg. W. Kurek 1952 (KRA); Trzebinia, roadside ditch near Chechło Reservoir (= Lake Chechelskie), leg. L. Olesiński 1970 (OLTC); Chrzanów, forest path, leg. K. Jędrzejko 1973 (KTU); Piła Kościelecka, meadow, leg. M. Piwowarczyk 1997 (KTU). DF58: Ojców National Park, Dolina Sąspowska (valley), wet meadow, leg. unknown 1965 (KRAM). DF65: Libiąż, wet pine-birch forest near railway embankment, leg. I. Mazaraki 1968 (KRAM). DF68: Łąki Wolskie near Kraków, leg. A. Rehmann 1869 (KRAM); Szczyglice, wet meadow, leg. A. Żmuda 1909 (KRAM); Szczyglice, wet meadow, leg. Lenewska 1990 (KRAM). DF69: Kraków, Wolski Forest, leg. T. Różycka 1953 (KRA). DF78: Skawina, meadow, leg. S. Chudoba 1957 (LOD); Wyźrał near Liszki, meadow, leg. D. Drozdowicz 1974 (KRA). DF79: Wola Duchacka, leg. I. Berdau 1859 (KRAM); Opatkowice near Kraków, leg. H. Trzcińska-Tacik 1986 (KRA). DF96: Miarkula glade, Leskowiec Mount, leg. St. Pelc 1954 (KRAM); Pod Wykopa glade, fen in groundwater seepage area, leg. St. Pelc 1958 (KRAM). DF97: Budzów, NNW of church, fen in groundwater seepage area in forest, 390 m a.s.l., leg. H. \& T. Tacik 1965 (KRAM). DF98: central part of Parszywka Mount, fen in groundwater seepage area below yellow tourist trail (crest) in S direction, leg. W. Bartoszek 1996 (KRA); nature reserve "Las Gościbia", near mouth of a tributary of Gościbia stream, on its right bank near Jaworze, 450 m a.s.l. leg. H. \& T. Tacik 1964 (KRAM); "Las Gościbia", fen in groundwater seepage area on SE slope, 460 m a.s.l., leg. H. \& T. Tacik 1965 (KRAM); Harbutowice, Podlesie, fen in groundwater seepage area, $430 \mathrm{~m}$ a.s.l., leg. H. \& T. Tacik 1965 (KRAM). DG07: Gorce, S slope of Borysówka, hay meadow near groundwater seepage area, leg. P. Irlik 1993 (KRAB); Maków Podhalański, meadow, leg. K. Guzik 2001 (KTU); Budzów, fen in groundwater seepage area, leg. D. Kempa 2005 (KRA). DG08: Górki, fen in groundwater seepage area, 432 m a.s.l., leg. B. Kotońska 1986 (KRA). DG09: Kokorzyk Mount (Fuck's Mount), above Skomielna Czarna, wetland near green tourist trail from Skomielna Czarna, $560 \mathrm{~m}$ a.s.l., leg. W. Bartoszek 1997 (KRA). DG15: Złotna, valley of Gawłowski Potok (stream), along stream edges, $670 \mathrm{~m}$ a.s.l., leg. K. Białecka 1971 (KRA); Szczawina, fen in groundwater seepage area, 1180 m a.s.l., leg. K. Białecka 1974 (KRA). DG16: Babia Góra, Markowe Równinki, leg. D. H. Zapałowicz 1877 (KRAM), Babia Góra, leg. T. Wilczyński 1911 (KRAM); Babia Góra (mount), glade near hostel, wetland, leg. J. Walas 1929 (KRAM); Babia Góra, on road through Ryzowane, leg. F. Krawiec 1930 (POZ); Zawoja, Markowy stream, 820 m a.s.l., leg. E. Małysa 1998 (KRA). DG17: Zawoja, forest, leg. B. \& L. Stuchlikowie 1955 (KRAM); Sucha Góra, meadow, leg. B. \& L. Stuchlikowie 1954 (KRAM). DG18: S of Toporzysko, moist alder wood, leg. M. Guzikowa 1966 (KRAM); Sidzina Mała, Wostojów Mount, W of its peak, wet meadow near spruce forest, $680 \mathrm{~m}$ a.s.l., leg. M. \& J. Guzikowie 1964 (KRAM); $0.5 \mathrm{~km} \mathrm{SW}$ of railway station in Jordanów, near road to Toporzysko, moist meadow, leg. M. \& J. Guzikowie 1966 (KRAM). DG27: Lipnica Wielka near Nowy Targ, damp places along stream, 755 m a.s.l., leg. M. Guzikowa 1963 (KRAM); Lipnica Wielka, between streams Kiczorka and Lizakówka, $750 \mathrm{~m}$ a.s.1.,leg. M. Guzikowa 1963 (KRAM); Lipnica Wielka, moist meadow, 845 m a.s.l., leg. M. Guzikowa 1963 (KRAM); Lipnica Wielka, Cyrak Mount, SE part, moist meadow, 795 m a.s.l., leg. M. Guzikowa (KRAM); Lipnica Mała near Nowy Targ, 
$\mathrm{N}$ end of village, wet meadow at bottom of Sykla stream valley, $750 \mathrm{~m}$ a.s.1., leg. M. Guzikowa 1966 (KRAM); Lipnica Mała, spruce forest edge, 745 m a.s.l., leg. M. Guzikowa (KRAM); Orawka near Nowy Targ, left-bank stream valley, Czarna Orawa, wet meadow, 720 m a.s.l., leg. M. Guzikowa 1963 (KRAM); Zubrzyca Górna, wet meadow E of church, 730 m a.s.1., leg. M. Guzikowa 1960 (KRAM). DG28: between Orawska and Podszkle, fen in groundwater seepage area near stream, $745 \mathrm{~m}$ a.s.1., leg. M. Guzikowa 1963 (KRAM); Podwilk, wet depression in a meadow, 690 m a.s.1., leg. M. Guzikowa 1966 (KRAM); Podwilk, wet meadow, 685 m a.s.l., leg. M. Guzikowa 1962 (KRAM); Podszkle, stream valley, ESE of church, wet meadow, 770 m a.s.l., leg. M. \& J. Guzikowie 1965 (KRAM); Podszkle, moist hay meadow at bottom of Bukowiński stream valley, 775 m a.s.l., leg. M. J. Guzikowie 1965 (KRAM); N of Podwilk, valley of left-bank tributary of Bąbeński stream, peaty meadow, 730 m a.s.1., leg. M. \& J. Guzikowie 1966 (KRAM); Harkabuz, upper part of village, S of little chapel near road to Bukowina, moist meadow near stream, leg. M. \& J. Guzikowie 1966 (KRAM). DG29: Pyzówka near Nowy Targ, wet meadow near railway station, 710 m a.s.1., leg. M. Guzikowa 1962 (KRAM); between Harkobuz and Raba Wyżna, moist roadside in spruce forest, 635 m a.s.1., leg. M. Guzikowa 1963 (KRAM). DG49: Ligasówki, fen in groundwater seepage area, leg. K. Chronowska 1955 (KRA); below Gubałówka peak, NW slope, fen in groundwater seepage area in forest, leg. K. Chronowska 1955 (KRA); meadow on Czarny Dunajec river, leg. K. Chronowska 1955 (KRA); Dzianisz, Tominów Wierch, wet meadow, leg. K. Chronowska 1955 (KRA); Furmanowa, wet meadow, leg. K. Chronowska 1955 (KRA); Podtatrze, on Czarny Dunajec river, near confluence of rivers Siwa and Kinowa Woda and Kojsówka, leg. J. Płazińska 1981 (KRAM). DG58: valley of Małe Koryciska, limestone outcrops in forest, 1000 m a.s.l., leg. A. Jasiewicz 1981 (KRAM); Tatra Mts, Mały Kopieniec, on limestone outcrops, in moist crevices, $1650 \mathrm{~m}$ a.s.l., leg. H. Czeczotowa (KRAM); Magura stream valley, Magura Witowska, peatland, 920 m a.s.l., leg. E. Koteja 1962 (KRAM); High Tatras, Wielki Kopieniec, SE of mountain pass to Mały Kopieniec, near road, leg. H. \& T. Tacik 1966 (KRAM). DG59: Dolina Strążyska (valley), leg. T. Chałubiński 1828 (ZAMU); Dolina Strążyska, roadside, leg. unknown 1926 (KRAM); Dolina Strążyska, leg. M. Bremówna 1937 (ZAMU); valley of Biały Potok (stream), leg. A. Wrześniewski 1838 (ZAMU); near stream on way from mountain pass between Grzybowska and Strążyska, leg. H. Piotrowska 1954 (POZ); near Głęboki stream, leg. E. Pancer 1955 (KRA); Mietłówka, S slope, fen in groundwater seepage area in forest, leg. E. Pancer 1955 (KRA); Dolina Małej Łąki (valley), wetland, leg. T. Tacik 1954 (KRAM); Dolina Małej Łąki, fen in groundwater seepage area, 980 m a.s.l., leg. R. Rajczak 1961 (KRA); Dolina Małej Łąki, 1050 m a.s.l., leg. B. Chwastowski 1977 (KRAM); Dolina za Bramką (valley), grassy stream edge, 920 m a.s.l., leg. R. Rajczak 1961 (KRA); Dolina za Bramką (valley), leg. nieznany 1904 (KRAM); Dolina Lejowa (valley), leg. A. Pacyna 1961 (KRA); near Spadowiec stream, below "Droga pod Reglami”, fir-spruce forest, patches of montane alder wood, 885-900 m a.s.1., leg. Z. Mirek 1985 (ZAMU); Zakopane, Polana Biały Potok (glade), 900 m a.s.l., leg. Z. Mirek 1989 (ZAMU), Dolina Lejowy Potok (valley), mire, leg. M. Jagiełło 1984 (KRAM); between Przysłop Miętusi and Kobylarz, thicket, 1200-1300 m a.s.1., leg. M. Pawlus \& A. Jasiewicz 1986 (KRAM); glade near tourist trail to Stoły, 1240 m a.s.l., leg. B. Chwastowski 1977 (KRAM); Mraźnica near Krzeptówki, slopes, near patches of shrubs leg. A. Jasiewicz 1981 (KRAM); Krzeptówki, fen in groundwater seepage area, leg. K. Zarzycki 1954 (KRAM); Krzeptówki, leg. K. Chronowska 1954 (KRAM); Tatra Mts, moist meadow near entrance to valley, leg. H. Czeczotowa (KRAM); Skibówki, leg. unknown (KRAM); Molkówka, peaty meadow, 970 m a.s.1., leg. H. \& T. Tacik 1978 (KRAM); Zakopane, “Droga pod Reglami”, leg. F. Berdau 1855 (KRAM); Zakopane, pasture near road to Dolina Białego (valley), leg. Zapałowicz (KRAM); Dolina Lejowa (valley), on slope, leg. A. Pacyna 1961 (KRAM); Dolina Kościeliska (valley), leg. A. Żmuda 1910 (KRAM); Łysanki, leg. unknown 1906 (KRAM). EF47: Wojkowa, leg. P. Paździk 1985 (KRAB); Wojkowa, moist meadow, leg. M. Piszczek 1987 (KRAB); Wojkowa, thicket, leg. M. Krupińska 1996 (KRAB). EF52: Rudno Dolne, wet meadow, leg. K. Towpasz 1995 (KRA). EF62: Niepołomice Forest, W part, leg. L. Nowak 1987 (KRAM). EF68: Wałki, wet forest, leg. M. Wayda 1990 (KRA). EF70: Bogucice, leg. J. Zabłocki 1922 (KRAM); Rżąka near Wieliczka, forest glade, leg. A. Żmuda 1909 (KRAM). EE71: Podłęże near Niepołomice, wet meadow, leg. A. Jasiewicz 1951 (KRAM). EF72: N of Grodkowice, drained meadows on fen, leg. A. Jasiewicz 1953 (KRAM); wetlands in Niepołomice Forest, leg. M. Zającówna 1953 (KRAM); Szarowskie Błoto, wasteland, leg. G. Piątek 2002 (KRAB). EF74: Jasień near Brzesko, leg. P. Zopser 1865 (KRAM). EF78: between Skrzyszów and Ładna, moist meadow, leg. K. Towpasz 1994 (KRA). EF87: Meszna Opacka, wet meadow, leg. K. Gołąb 2003 (KRA). EF90: Poręba, forest glade, 450 m a.s.1., leg. W. Bartoszek 1992 (KRA); Poręba, peaty glade in forest, leg. W. Bartoszek 1993 (KRA); Chełmiec Mount near Lipnik, fen in groundwater seepage area near forest edge, 530 m a.s.l., leg. W. Bartoszek 1994 (KRA); between Lipnik and Wiśniowa, thicket along stream, 430 m a.s.l., leg. W. Bartoszek 1994 (KRA); between Lipnik and Wiśniowa, meadow, wet site at bottom of N slope of Pasternik Mount, 425 m a.s.l., leg. W. Bartoszek 1994 (KRA); E part of Chełmiec Mount, groundwater seepage area, 540 m a.s.1., leg. W. Bartoszek 1994 (KRA); Pcim Bornia, wetland, leg. K. Starmach 1926 (KRAM); Pcim, leg. K. Starmach 1925 (KRAM). EF93: Żmiące-Zagnaniec, leg. M. Szewczyk 1994 (KRAM). EG02: between Mogielnica and Krystonowo, 850 m a.s.l., meadow, leg. K. Towpasz 1969 (KRA); Mogielica (Chyrzówki), NW slope, near stream, 750 m a.s.l., leg. K. Towpasz 1966 (KRA); Łopień, NW slope, fen in groundwater seepage area, leg. K. Towpasz 1966 (KRA); Łopień, NW slope, near stream, 500 m a.s.1., leg. K. Towpasz 1966 (KRA); Paproć, fen in groundwater seepage area, 600 m a.s.1., leg. K. Towpasz 1969 (KRA). EG03: Stara Wieś, near stream, 420 m a.s.1., leg. K. Towpasz 1969 (KRA); Island Beskids (Beskid Wyspowy Mts), Jaworza mountain range, leg. J. Staszkiewicz 1966 (KRAM). EG04: Tęgoborze, leg. J. Staszkiewicz 1966 (KRAM); Ujanowice, fen in groundwater seepage area, leg. M. Szewczyk 1978 (KRAM). EG05: wetland, leg. A. Środoń 1946 (KRAM). EG09: Pagorzyna, wet old field, leg. K. Oklejewicz 1989 (KRA). EG10: Luboń Wielki, meadow near 
blue tourist trail, 1022 m a.s.l., leg. unknown 1966 (KRA); Sienno, rye field, leg. unknown 1978 (KRA). EG12: Mogielnica, NW slope, fen in groundwater seepage area, 770 m a.s.l., leg. K. Towpasz 1967 (KRA). EG14: Królówki, moist meadow, leg. K. Ciońćka 1993 (KRA); Wysokie-Kaczkówka, fen in groundwater seepage area, leg. M. Szewczyk 1997 (KRA). EG15: Wólki, Nowy Sącz, leg. B. Pawłowski 1919 (KRAM). EG18: Czernichów, meadow, 430 m a.s.1., leg. B. Kotońska 1980 (KRA). EG19: Ropica Dolna, meadow, leg. S. Załęski 1978 (KRA); Przegonina near Gorlice, wet meadow, leg. K. Oklejewicz 1994 (*); Bednarka, wet wastelands, leg. K. Oklejewicz 1994 (*). EG20: Obidowa, Gorce, leg. H. Trzcińska-Tacik 1970 (KRA); Obidowa, wet meadow, leg. St. Cabała 1973 (KTU); Jaworzyna Obidowska glade near Turbacz, leg. B. Chwastowski 1980 (KRAM). EG21: Turbacz, Hala Długa (alp), fen in groundwater seepage area, leg. J. Kornaś 1953 (KRA); Borysówka glade, Gorce Mts, wet meadow, leg. M. Burzyk 1993 (KTU); Polana Średniak (glade), wet meadow, leg. M. Golinia \& A. Saszko 2003 (LOD); Polana Brożek (glade), 950 m a.s.l., leg. B. Chwastowski 1980 (KRAM); groundwater seepage area above Ustrzyk, near Ochotnica Górna, leg. J. Staszkiewicz 1951 (KRAM). EG23: Zarzecze, moist meadow, 360 m a.s.l., leg. K. Stawowczyk 2007 (KRA); Błyszcz, fen in groundwater seepage area, 900 m a.s.1., leg. K. Stawowczyk 2007 (KRA); Wdziany Niżna, fen in groundwater seepage area, 720 m a.s.1., leg. K. Stawowczyk 2007 (KRA). EG24: Gołkowice, leg. J. Michalska 1967 (TRN); Moszczenica Wyżna, moist meadow, 380 m a.s.l., leg. K. Stawowczyk 2007 (KRA); Gaboń, moist meadow, leg. K. Stawowczyk 2007 (KRA). EG27: Krzyżówka, part N, wet meadow, leg. K. Oklejewicz 1999 (*). EG29: Smerekowiec, wet meadow, leg. K. Oklejewicz 1994 (*); Wołowiec, roadside, leg. K. Oklejewicz 1994 (*); Banica, wet wastelands, leg. K. Oklejewicz 1999 (*). EG31: Łopuszna, fen in groundwater seepage area, leg. A. Magiera 1982 (KRAB); Dolina Białki (valley), between bridge from Nowa Biała to Krempachy and confluence of Białka and Dunajec rivers, leg. J. Płazińska 1981 (KRAM); Frydman, fen in groundwater seepage area, leg. K. Grodzińska 1977 (KRAM); field roadside, between Frydman and Fałtysz, leg. K. Grodzińska 1968 (KRAM); fen in groundwater seepage area near Frydniański stream, leg. K. Grodzińska 1964 (KRAM); Pieniny Rock Belt (Pieniński Pas Skałkowy), leg. K. Grodzińska 1967 (KRAM); Krempachy, near stream, leg. K. Grodzińska 1967 (KRAM); Pieniny Rock Belt, Hombark mountain range, leg. K. Grodzińska 1963 (KRAM); Skalice Spiskie, Stajnie Hunaj, fen in groundwater seepage area, leg. K. Grodzińska 1964 (KRAM). EG32: Spisz Foothills (Pogórze Spiskie), wetland, 580 m a.s.l., leg. A. Nikel 2001 (KRAM); Gorce Mts, Polana Brożek (glade), 950 m a.s.1., leg. K. Grodzińska 1963 (KRAM); Zielone Skały, Pieniny Rock Belt, leg. K. Grodzińska 1963 (KRAM). EG33: Krościenko, wet meadow near stream, leg. L. Fagasiewicz 1950 (LOD); Krościenko, river bank, leg. K. Stawowczyk 2008 (KRA); slopes of Ociemny Wierch, Pieninki mountain range, leg. H. Piotrowska 1969 (UGDA); Dunajec river valley, between Szczawnica and Łącica, fen in groundwater seepage area on river bank, leg. S. Pelc 1969 (KRA); Złotne, wet meadow, leg. A. Lisik 1993 (KTU); Bereśnik, SE slope, moist place near road, 700 m a.s.1., leg. K. Stawowczyk 2006 (KRA); Stajkowa Góra (mount) near Krościenko, N slope, fen in groundwater seepage area, 550 m a.s.1., leg. K. Stawowczyk 2006 (KRA). EG34: Wąwóz Homole (gully), fen, leg. A. Sendek 1972 (KRA); Wąwóz Homole, moist meadow at valley bottom, leg. A. Jasiewicz 1968 (KRAM); Biała Woda, fen in groundwater seepage area, leg. A. Jasiewicz 1968 (KRAM); Rohacz stream valley, damp places along stream, leg. K. Stawowczyk 2007 (KRA); Roztoka Wielka stream valley, 720 m a.s.l., leg. K. Stawowczyk 2007 (KRA); Czercz stream valley, fen in groundwater seepage area, leg. K. Stawowczyk 2007 (KRA); valley of Kotelnicki Potok (stream), damp places along road, 730 m a.s.1., leg. K. Stawowczyk 2006 (KRA); valley of Stary Potok (stream), moist place near road, 600 m a.s.l., leg. K. Stawowczyk 2006 (KRA); Rusinowski Wierch (mount), SE slope, fen in groundwater seepage area, 800 m a.s.1., leg. K. Stawowczyk 2006 (KRA); ); Gabańka, fen in groundwater seepage area, 800 m a.s.1., leg. K. Stawowczyk 2005 (KRA); Sielski Potok, fen in groundwater seepage area, 670 m a.s.l., leg. K. Stawowczyk 2007 (KRA); Hurcałki 938 (mount), W slope, meadow, 900 m a.s.l., leg. K. Stawowczyk 2007 (KRA). EG35: Piwniczna Zdrój, fen in groundwater seepage area, 620 m a.s.1., leg. K. Stawowczyk 2007 (KRA); Obłazy Ryterskie, moist place on a path, leg. K. Stawowczyk 2007 (KRA). EG37: Mochnaczka Wyżna near Krynica, wet wastelands, leg. K. Oklejewicz 1994 (*); Krynica, wet meadow, leg. E. Krasicka-Korczyńska 1994 (BYDG); Tylicz-Muszynka, fen in groundwater seepage area, leg. M. Szewczyk 1995 (KRA). EG38: Hańczowa, wet meadow, leg. K. Oklejewicz 1994 (*). EG40: right bank of Biały Dunajec near Poronin, damp depression, leg. K. Zarzycki 1954 (KRAM). EG41: S of Bukowina Tatrzańska, fen in groundwater seepage area, leg. A. Jasiewicz 1952 (KRAM). EG47: Powroźnik, Młynne stream valley, flooded sites, leg. B. Zemanek 1970 (KRA). EG50: Bugówka near Kuźnice, meadow, leg. L. Fagasiewicz 1955 (LOD); Dolina Bystrej (valley), wet meadow, 1060 m a.s.l., leg. H. Piękoś 1961 (KRA); near road to ski lift to Nosal Mount, waterlogged site covered with rubble, leg. Z. Radwańska-Paryska 1981 (ZAMU); Myślenickie Turnie, 1350 m a.s.l., leg. N. Biatokoz 1996 (KTU); Toporowy Staw Wyżni, fen in groundwater seepage area, leg. H. \& T. Tacik 1966 (KRAM); Toporowy Staw Wyżni (pond), leg. unknown 1914 (KRAM); Chłabówka, wetland surrounded by spruce forest, leg. H. \& T. Tacik 1966 (KRAM); Tatrzańska Dolina (valley) near Bielski Potok (stream), 730 m a.s.l., leg. A. Jasiewicz 1955 (KRAM); Dolina Białego (valley), Western Tatras, leg. B. Chwastowski 1977 (KRAM); Zakopane, Bystra river valley, moist meadow, 950 m a.s.l., leg. H. Piękoś (KRAM); Zakopane, Bogówka, meadow, leg. M. Sychowa 1955 (KRAM). EG60: Morskie Oko Lake, mire, leg. uknown 1926 (KRA); Morskie Oko, mire, leg. B. Pawłowski 1926 (KRAM); scree near Morskie Oko (lake), leg. J. Błaszczyk 1937 (KRA); slope of Miedziane Mount opposite to Mnich Mount, grasslands on granite, $1650 \mathrm{~m}$ a.s.1., leg. A. Jasiewicz 1974 (KRAM); Miedziane Mount, edge of bog, 1480 m a.s.l., leg. A. Jasiewicz 1975 (KRAM); Marchwiczny Żleb (couloir) leg. Z. Radwańska-Paryska 1949 (KRAM). FG20: Nieznajowa, Wisłoka river valley, alluvial forest, leg. M. Zarzyka 1998 (KRA); Ponice, waterlogged bank of Poniczanka, leg. E. Stanisz 2006 (KRA). FG30: Radocyna, Wisłoka river valley, 
meadow, leg. M. Zarzyka 1999 (KRA); Dębi Wierch, source of Wisłoka river, fen in groundwater seepage area, leg. M. Zarzyka 1999 (KRA).

Mazowieckie. DC46: Blinno, leg. Kępczyński 1961 (TRN). DC48: Lutocin, leg. K. Kępczyński 1961 (TRN). DC56: Lipno on Skrwa river, leg. A. Ejsmond 1885 (WA). DD06: Misiadła, meadow, leg. H. Kaczorowska 1957 (LOD); Gostynin Rataje, mire, leg. J. Kowalska 1959 (LOD). DD09: Studzieniec near Sanniki, leg. K. Drymmer 1995 (WA). EC37: Józefowo, moist meadow, leg. J. Gałązka 1961 (LOD). EC48: Perzanowo, sandy meadow, leg. J. Tomczak 1985 (WSRP). EC58: Łaś, moist meadow, leg. E. Rutkowska 1977 (WSRP). EC96: Wieliszew, wet old field, leg. H. Romanowska 1967 (LOD). EC99: Kurówka, meadow, leg. Z. Głowacki 1980 (WSRP). ED06: Jabłonna, meadow, leg. R. Kobendza \& M. Zamara 1947 (WA). ED13: Wiejca, meadow, leg. R. Kobendza 1925 (WA); Korfowe, fen, leg. Z. Głowacki 1990 (WSRP); Koszówka, fen, leg. Z. Głowacki 1990 (WSRP). ED15: Warsaw, Bemowo Forest (Las Bemowski), moist meadow leg. I. Kirpluk 1986 (WA). ED16: Warszawa Buraków, leg. R. Kobendza \& M. Zanowa 1947 (WA). ED17: Warszawa Rembertów, mire, leg. M. Andrzejewska 1975 (LOD). ED19: Feliksów, fen, leg. Z. Głowacki 1990 (WSRP). ED26: Warszawa Ujazdów, leg. R. Kobendza \& M. Zanowa 1946 (WA). ED27: Warszawa Międzylesie, moist forest, leg. T. Małysz 1979 (WA). ED35: Pęcice, mire, leg. M. Gągała 1982 (WA). ED39: Sępochów, fen, leg. Z. Głowacki 1991 (WSRP). ED48: Całowanie, leg. J. Zielińska 1951 (WA); Całowanie, moist meadow, leg. M. Małecka 1959 (WA); Całowanie, moist mixed deciduous forest on peat, leg. K. Nowak 1962 (WA). ED57: Czachówek, wet meadow, leg. Z. Głowacki 1978 (WSRP). EE36: 5 km NNE of Szydłowiec, peaty meadow, leg. M. Nobis 2003 (KRA); 1 km S of Rogowskie Komorniki, ditch in farmland leg. M. Nobis 2004 (KRA). EE37: Pakosław near Iłża, poor fen, leg. T. Tacik 1954 (KRAM). EE38: Pakosław, poor fen, leg. H. Trzcińska-Tacik 1954 (KRA), leg. B. Ritras 1954 (KRA); Płudnica, moist meadow, leg. M. Nobis 2003 (KRA). EE45: Majdów, nature reserve "Cisowy Majdów", mire, leg. J. Grygiel \& E. Bróż 1976 (KTC). EE47: Zbijów Mały, peaty meadow, leg. M. Nobis 2002 (KRA). FC74: Garnek, waterlogged edge of meadow, leg. Z. Głowacki 1984 (WSRP); Garnek, leg. P. Kalinowski 2010 (*); Nowa Wieś, moist field, leg. Z. Głowacki 1989 (WSRP); Nowa Wieś, moist forest, leg. Z. Głowacki 1989 (WSRP). FC81: Łochów, mire, leg. Z. Głowacki 1982 (WSRP). FC82: Lipki Stare, fen, leg. P. Krzyżanowski 1976 (WSRP). FC86: Kolonia Wieska, leg. P. Kalinowski 2013 (*); Wieska N, leg. P. Kalinowsko 2013 (*). FC90: Dębe Małe, fen, leg. Z. Głowacki 1978 (WSRP). FC91: Myszadła, peaty soil, leg. anonymous 1860 (LOD). FC93: Poszewka, moist meadow, leg. Z. Głowacki 1982 (WSRP). FC96: Wirów, meadow, leg. Z. Głowacki 1986 (WSRP); Kolonia Jabłonna Lacka, leg. Kalinowski 2013 (*). FD03: Ruchna, forest clearing, leg. C. Krasnodębska 1976 (WSRP). FD07: SE of Korczew, wet meadow, leg. Z. Głowacki 1985 (WSRP); Kamianki Czabaje, leg. P. Kalinowski 2004 (*); Korczew NE, leg. P. Kalinowski 2006 (*); Szczeglacin-Podknychowie, leg. P. Kalinowski 2009 (*); Korczew N, leg. P. Kalinowski 2009 (*); Laskowice NE, leg. P. Kalinowski 2010 (*); Laskowice-Kamianki, forest, leg. P. Kalinowski 2011 (*); Zaleś, leg. P. Kalinowski 2013 (*). FD08: Ruska Strona, leg. P. Kalinowski 2004 (*); Tokary, leg. P. Kalinowski 2011 (*); Góry NW, leg. P. Kalinowski 2013 (*). FD14: Kisielany-Kuce, leg. P. Kalinowski 2013 (*). FD16: Uziębły, small glades in wet forest, leg. Z. Głowacki 1974 (WSRP). FD18: Łosice, fen, leg. F. Karo 1873 (WA); Łosice, leg. Z. Krygier 1976 (WSRP); Myszkowice, meadow, leg. Z. Głowacki 1974 (WSRP), leg. B. Wyczółkowska 1976 (WSRP); Niemojki, moist meadow, leg. B. Wyczółkowska 1976 (WSRP); Zakrze, fresh mixed coniferous forest, leg. Z. Krygier 1976 (WSRP). FD23: Sionna, wetland surrounded by forest, leg. Z. Głowacki 1974 (WSRP). FD25: Golice, fen in groundwater seepage area near ponds, leg. G. Jówko 1973 (LBLM); Golice, fen in groundwater seepage area near ponds, N of village, leg. Z. Głowacki 1973 (WSRP); Rakowiec, stream valley near Kolonia Żelkowa, leg. Z. Głowacki 1974 (WSRP); Kolonia Strzała, fen, leg. Z. Głowacki 1975 (WSRP). FD26: Krzymosze, wet forest glade, leg. Z. Głowacki 1974 (WSRP). FD27: Mordy, fen, leg. Z. Głowacki 1978 (WSRP). FD30: Siennica, meadow, leg. B. Dębowska, 1970 (LOD); Tartak Prusy, fen in groundwater seepage area, leg. Z. Głowacki 1979 (WSRP). FD31: Kiczki, meadow, leg. L. Komorek 1955 (LOD). FD35: Ujrzanów, edge of drainage ditch, leg. Z. Głowacki 1974 (WSRP); Białki Siedleckie, overgrown sites of peat extraction, leg. Z. Głowacki 1974 (WSRP); Siedlce-Piaski, fen, leg. Z. Głowacki 1985 (WSRP). FD36: NE of Rówce, fen in groundwater seepage area, leg. Z. Głowacki 1973 (WSRP); Zbuczyn, drainage ditch, leg. A. Zbucka 1977 (WSRP). ). FD50: Pilawe, forest, leg. B. Rosłaniec no date (WSRP). FD81: S of Oronne, fen surrounded by forest, leg. Z. Głowacki 1984 (WSRP).

Opolskie. CE79: Kolonia Biskupska near Olesno, most meadow, leg. Z. Kącki \& Z. Dajdok 1999 (WRSL). CE88: E of Osiecko, wooded patch among meadows, leg. E. Kuźniewski 1959 (SZUB). CE95: Czarnowąsy, near railway tracks, leg. S. Michalak 1969 (OPOL). CF05: Groszowice, leg. K. Bialucha 1938 (OPOL); Opole, leg. K. Bialucha 1939 (OPOL). CF09: Liszczak, pond edge, leg. G. Chmiel 1977 (KTU). CF14: Bory Niemodlińskie, roadside, leg. W. Głombica 1978 (KTU). CF16: Ligota Dolna, meadow near N slope of Kamienna Góra, leg. S. Michalak 1968 (OPOL). CF32: Prudnik, leg. Barthel 1884 (TRN). CF34: Głogówek, moist meadow, leg. P. Szotkowski \& A. Urbisz 1977 (KTU).

Podkarpackie. EF79: Kamienica Dolna, sedge bed, leg. K. Towpasz 1976 (KRA). FE84: Zaklików, meadow, leg. H. Koporska 1926 (LBLM). FF16: SE of Bukowina, moist meadow, leg. A. Michalewska 2003 (KRA); Rędziny-Sibigi, meadow, leg. A. Michalewska 2003 (KRA); S of Ryczki, meadow, leg. A. Michalewska 2004 (KRA); 1.5 km NW of W end of Ryczki, meadow, leg. A. Michalewska 2004 (KRA). FF17: $750 \mathrm{~m} \mathrm{~S}$ of W end of Harasiuki, moist forest roadside, leg. A. Michalewska 2004 (KRA); SW of Huciska, moist meadow, leg. A. Michalewska 2003 (KRA). FF18: S of W end of Harasiuki, near a watercourse, leg. A. Michalewska 2004 (KRA); close to housing estate near brickyard Harasiuki, moist meadow, leg. A. Michalewska 2004 (KRA). FF24: Zalesie, swamp in forest, leg. G. Dziundzio 1977 (KRA). FF27: E of Łazów, moist meadow, leg. A. Michalewska 2003 (KRA); Stary Jasiennik, moist meadow, leg. A. Michalewska 2004 (KRA); NE of S end of Łazów, 
moist meadow near drainage ditch, leg. A. Michalewska 2004 (KRA). FF28: W of Jastrzębiec, ditch, leg. A. Michalewska 2003 (KRA). FF35: Turza, ditch edge, leg. Kołodziej 1992 (KRAB). FF40: Lączki Brzeskie, fen, leg. C. Brzostowska 1977 (KRA). FF48: Kępa, sand pit near settlement, close to oxbow lake of San river near village Pigany, leg. A. Michalewska \& M. Nobis 2004 (KRA). FF58: Ujezna near Przeworsk, meadow, leg. unknown 1971 (KRA); Jagiełła near Przeworsk, Trojany fen, leg. M. Nowiński 1924 (KRAM). FF61: Chechły, moist meadow, leg. K. Towpasz 1980 (KRA). FF63: Nosówka, sedge bed, leg. K. Towpasz 1980 (KRA). FF67: Dębów, wet meadow, leg. G. Zięć 2007 (KRA). FF73: Nowa Wieś Czadecka, eutrophic fen in groundwater seepage area, leg. Kucharczyk 1987 (KRAB). FF80: Wola Brzostecka, sedge bed, leg. K. Towpasz 1976 (KRA). FF87: Przedmieście Dubieckie near Dynów, mire, leg. B. Chwastowski 1978 (KRAM). FF89: Zasanie near Przemyśl, wet meadow, leg. unknown 1876 (KRAM); Kozownica, wet meadow, leg. unknown 1877 (KRAM). FF91: Niepla, moist meadow, leg. K. Towpasz 1980 (KRA). FF93: Lutcza, wet meadow, leg. B. Gutkowska 2010 (KRA). FF95: Przysietnica, wet meadow, leg. B. Gutkowska 2009 (KRA); Wesoła, wet meadow, leg. B. Gutkowska 2004 (KRA). FG01: Tarnowiec part W, wet meadow, leg. K. Oklejewicz 1990 (KRA). FG02: Leśniówka, wet meadow, leg. K. Oklejewicz 1990 (KRA); Żarnowiec, marshy gravel pit near Jasiołka, leg. K. Oklejewicz 1990 (KRA). FG03: between Widacz and Haczów, fen at edges of waterhole leg. K. Oklejewicz 1990 (KRA). FG05: Obarzym, pond edge, leg. B. Gutkowska 2008 (KRA). FG11: Siedliska Żmigrodzkie, wastelands, leg. K. Oklejewicz 1990 (KRA); Głosce, S part, wet wastelands, leg. K. Oklejewicz 2000 (*); Kąty, Wisłoka river bank, leg. M. Zarzyka 1999 (KRA). FG12: Łąki Dukielskie part S, wet meadow, leg. K. Oklejewicz 2009 (*). FG13: Rymanów Zdrój, moist fir forest, leg. K. Piech 1925 (KRAM). FG16: Sanok, meadow near cemetery, leg. K. Piech 1922 (KRAM). ). FG21: Dobańce, wet meadow, leg. K. Oklejewicz 2000 (*); Krempna near Nowy Żmigród, S of research station of Jagiellonian University, meadow near stream, leg. A. Nobis \& M. Nobis 2006 (KRA); Ropianka (crossroads), site where wood was stored, leg. K. Oklejewicz $2000\left(^{*}\right)$; Ropianka, waterlogged site near road in fir forest, leg. K. Oklejewicz 1999 (*). FG22: Dukla, E of Kamienna Mount, leg. K. Pstrokowska 1955 (KRA); Cergowa Mount near Dukla, meadow on NE slope, leg. K. Oklejewicz $1994\left(^{*}\right)$; Lipowica quarry, gravelly patch in beech forest, leg. K. Oklejewicz, $2010(*)$; Low Beskids, Cergowa Mount, N slope, peatland, leg. T. Tacik 1955 (KRAM). FG23: Zawadka Rymanowska, E part, edge of fish pond, leg. K. Oklejewicz 1999 (*); Głębokie, wet wastelands, leg. K. Oklejewicz 1994 (*); between Wisłoczek and Puławy Dolne, wet wastelands, leg. K. Oklejewicz 1994 (*). FG24: Darów Forest Range, N part, wet site where wood was stored, leg. K. Oklejewicz 2000 (*); Puławy Górne, wet meadow, leg. K. Oklejewicz 1994 (*); Wola Piotrowa, ditch in a meadow, leg. K. Oklejewicz 1994 (*); Puławy Górne, wet wastelands, leg. K. Oklejewicz 1994 (*); Smokowiska Mount, SW part, wet wastelands, leg. K. Oklejewicz 2000 (*); Żebracze, edge of pine forest, leg. K. Oklejewicz 2000 (*); between Tarnawka and Rudawka Rymanowska, leg. K. Grodzińska 1959 (KRAM); Kiczerka near Rudawka Rymanowska, leg. K. Grodzińska 1959 (KRAM). FG30: Ożenna, Magura National Park, SW part, forest edge, leg. M. Szewczyk 1998 (KRAM). FG34: Berdo Mount, S part, waterlogged, rocky bank of Wisłok river, leg. K. Oklejewicz 2000 (*); Darów, wet wastelands, leg. K. Oklejewicz 1999 (*); Tokarnia Mount, wet wastelands, leg. K. Oklejewicz 1994 (*); Moszczaniec near Sanok, wetland near stream, leg. K. Piech 1931 (KRAM). FG45: W of Komańcza, wet wastelands, leg. K. Oklejewicz 1994 (*); Komańcza, edge of forest stream, leg. J. Skrabut 1996 (KRAB). FG46: Bystre near Baligród, fen in groundwater seepage area, 500 m a.s.1., leg. A. Jasiewicz 1962 (KRAM); Rabe, meadow on Żebrak, leg. K. Piech 1927 (KRAM). FG55: Łupków, fen in groundwater seepage area near road to Smolniki, leg. H. Trzcińska-Tacik 1989 (KRA). FG56: Sasów near Cisna, fen in groundwater seepage area, 960 m a.s.1., leg. A. Jasiewicz \& K. Zarzycki 1954 (KRAM). FG57: Babkowce, on slope of Jabłońska Góra (mount), 750 m a.s.l., leg. J. Wójcicki 1980 (KRAM). FG59: Stuposiany, leg. M. Rejewski 1969 (TRN); Stuposiany, fen in groundwater seepage area, 560 m a.s.1., leg. A. Jasiewicz 1960 (KRAM). FG68: Wetlina, forest edge, leg. J. Cygler (KRAB); Wetlina, steep bank of Wetlinka river, leg. H. Więcław 2010 (**); Wetlina, fen in groundwater seepage area, leg. B. Chwastowski 1978 (KRAM); Moczarne near Wetlina, moist meadow in Solinka river valley, leg. A. Jasiewicz 1961 (KRAM); Moczarne, fen in groundwater seepage area near a lodging house for workers, leg. A. Jasiewicz 1961 (KRAM). FG69: Wyżniański Wierch, fen in groundwater seepage area, leg. K. Zarzycki 1958 (KRAM); Połonina Caryńska, depression with standing water, leg. H. Więcław $2010\left(^{*}\right)$; Brzegi Górne, roadside ditch, leg. H. Więcław 2010 (**). FG79: Wołosate, roadside ditch, leg. H. Więcław 2010 (**); N of Wołosate, meadow, leg. H. Więcław 2010 (**). GF51: between Bukowina and Czerniawka, leg. M. Raciborski 1910 (KRA). GF71: Starzawa-Nakło, meadow, leg. B. Kotula 1878 (KRAM). GG40: 400 m SW of Michniowiec, meadow near a watercourse, leg. M. Nobis 2005 (KRA). GG60: Lutowiska, Terebowiec stream valley, leg. K. Rostański 1988 (KTU); Terebowiec stream valley, meadow near asphalt road, 750 m a.s.l., leg. G. Zagórny 1990 (LBLM, BNPH); Terebowiec stream valley, leg. B. Chwastowski 1976 (KRAM); rock outcrops under Krzemień Mount, 1200 m a.s.l., leg. St. Kucharzyk 1991 (BNPH). GG70: S slope of Szeroki Wierch (mount), leg. K. Zarzycki 1958 (KRAM); Wołosate, Przełęcz Beskid (mountain pass), roadside ditch, leg. H. Więcław $2010(* *)$; Wołosate, forest roadside in direction of Szczawinki river, leg. H. Więcław 2010 (**); Przełęcz Bukowska (mountain pass), meadow along stream, leg. H. Więcław 2010 (**).

Podlaskie. FA78: Sudawskie, meadow, leg. A. Sokołowski 1969 (BIL). FA88: Bondziszki, mire, leg. K. Sokołowska 1969 (BIL); Jaczno, mire, leg. K. Sokołowska 1969 (BIL); Smolniki, Jaczno Lake, leg. A. Kawecka 1980 (BIL); NE of Potopy, pasture, leg. H. Więcław 2010 (**). FA89: Becejły, edge of Lake Iłagiel, leg. A. Sokołowski 1969 (BIL). FA94: Tatary Mts, meadow, leg. J. Kozak 1970 (BIL). FA97: Bachanowo near Hańcza, springhead near Czarna Hańcza river, leg. A. Sokołowski 1975 (BIL); Bachanowo near Hańcza, alder thicket on a meadow, leg. S. Fieńko 1969 (BIL); Szeszupka, peaty meadow, leg. S. Fieńko 1969 (BIL); Szeszupka, poor fen, leg. A. Sokołowski 1981 (BIL); Turtul, fen, leg. A. Kawecka 1981 (BIL); peat- 
land near Lake Linówek, leg. K. Stawowczyk 2004 (KRA). FA98: Sidorówka, flooded peat pit, leg. A. Kawecka 1969 (BIL); Szurpiły, partly overgrown lake, leg. A. Kawecka 1969 (BIL); Udryn, willow thicket, leg. A. Kawecka 1969 (BIL); Udryn, meadow, leg. A. Kawecka 1980 (BIL); Wodziłki, meadow, leg. S. Fieńko 1969 (BIL); Łopuchowo, wet meadow, leg. P. Naks 2004 (KRA). FB06: Filipów Pierwszy, moist meadow at wet deciduous forest edge in Rospuda river valley, leg. A. Pliszko 2008 (*). FB07: Skazdub Stary, meadow, S. Fieńko 1969 (BIL). FB08: Osowa, fen in Czarna Hańcza river valley, leg. A. Pliszko 2010 (KRA). FB09: Piertanie, meadow, leg. A. Kawecka 1969 (BIL). FB16: Gębalówka, wet coniferous forest with former sites of peat extraction, leg. A. Pliszko 2011 (KRA). FB18: Krukówek, alluvial forest, leg. A. Kawecka 1970 (BIL). FB27: Podwronowo, leg. A. Kawecka 1970 (BIL). FB28: Święte Miejsce, edge of Lake Jałowo, leg. A. Kawecka 1970 (BIL). FB38: Wypusty, alder carr, leg. J. Żurawski 1971 (BIL); Żarnowo, wet meadow, leg. J. Żurawski 1971 (BIL). FB39: Augustów, leg. P. Kalinowski 2008 (*). FB49: Białobrzegi, alder carr, leg. J. Żurawski 1971 (BIL). FB58: Czarna Wieś, alder carr, leg. uknown 1956 (BIL). FB67: Brzózki Ciszewskie near village Sołki, birch forest edge, leg. A. Wyszkowska 1979 (LOD). FB74: Szczuczyn, waterlogged meadow, leg. A. Chodnicki 1978 (WSRP). FB87: Downary, meadow, leg. J. Żurawski 1971 (BIL); Rybaki, alluvial forest, leg. A. Kawecka 1973 (BIL). FB88: Krzeczkowo, alluvial forest and ditch, leg. A. Kawecka 1973 (BIL). FB93: Stawiski, moist meadow in Dzierzba river valley, leg. A. Sokołowski 1968 (BIL). FC13: Piątnica, fen in Narew river valley, leg. H. Gierłowska 1982 (WSRP). FC26: Wnory-Wiechy near Łapy, mire, leg. A. Sokołowski 1977 (BIL). FC39: Borowskie Gziki, alder carr, leg. A. Kawecka 1970 (BIL). FC69: Kalnica, meadow, leg. K. Sokołowska 1970 (BIL). FC98: Zajęczniki, leg. P. Kalinowski 2013 (*). FC99: Siemiatycze, wet meadow, leg. E. Ćwikliński 1985 (WSRP). GA90: Kompocie, leg. A. Kawecka 1969 (BIL); Smolany, wet meadow, leg. S. Fieńko 1969 (BIL). GB00: Aleksandrowo, meadow, leg. A. Kawecka 1969 (BIL); Krasnopol, wet meadow, leg. S. Fieńko1969 (BIL); Pawłówka, pasture, leg. S. Fieńko1969 (BIL); 0.5 km W of Remieńkiń, thicket, leg. A. Kawecka 1969 (BIL); Remieńkiń, meadow at N edge of Lake Dowcień, leg. A. Kawecka 1969 (BIL); Romanowce, Czarna river valley, leg. S. Fieńko 1969 (BIL); Stabieńszczyzna, meadow, leg. A. Kawecka 1969 (BIL); Żubrówka, meadow, leg. A. Kawecka 1969 (BIL). GB01: Dworczyska, mire, leg. J. Żurawski 1971 (BIL); Sejny, ditch, leg. K. Dyga 1976 (WA); Berżniki, forest on NW edge of Lake Gajlik, leg. E. Jabłońska 2001 (WA); peatland on E edge of Lake Gajlik, leg. E. Jabłońska 2002 (WA). GB02: Folwark, willow thicket, leg. A. Kawecka 1969 (BIL); Berżniki, meadow, leg. H. Więcław 2010 (**). GB11: Giby, meadow on peaty soil at N edge of Lake Kaczan, leg. T. Szarejko 1991 (OLTC); Giby, peatland near Lake Dumbel, leg. H. Werblan-Jakubiec 1975 (WA). GB21: Gorczyca, poor fen, leg. J. Żurawski 1971 (BIL); Płaska, alder carr, leg. J. Żurawski 1974 (BIL). GB22: Muły, mire, leg. A. Sokołowski 1972 (BIL). GB30: Sucha Rzeczka, mire, leg. A. Sokołowski 1971 (BIL). GB31: Mikaszówka, mire, leg. J. Żurawski 1971 (BIL). GB32: Gruszki, wet meadow, leg. J. Żurawski 1971 (BIL); Rubcowo, forest, leg. J. Żurawski 1974 (BIL). GB41: Lipsk, meadow, leg. J. Żurawski 1974 (BIL). GB61: Nowinka, moist forest meadow, leg. A. Dackiewicz 1969 (BIL). GB63: Sterpejki, wet meadow, leg. B. Krawel 1982 (WSRP). GB81: Rudawka, forest meadow, leg. S. Fieńko 1969 (BIL). GB83: Malawicze, meadow, leg. J. Pietrzykowski 1969 (BIL). GB90: Brzozówka Koronna, ditch in farmland, leg. N. Romańczuk 1973 (BIL); S of village Karczmisko, Czarna Rzeczka stream valley, leg. A. Sokołowski 1989 (BIL). GB92: Lipina, alder carr and ditch in farmland, leg. N. Romańczuk 1973 (BIL); Złota Wieś, forest section 108, clearing (former mixed coniferous forest), leg. A. Kawecka 1963 (BIL). GB93: Kozłowy Ług, grassy border between fields, leg. A. Sokołowski 1969 (BIL); Talkowszczyzna, meadow, leg. S. Fieńko 1969 (BIL); Trzciano Nowe, meadow, leg. A. Sokołowski 1969 (BIL). GB94: Krynki, moist meadow, leg. A. Oleksza 1982 (WSRP). GC01: Horodnianka, meadow near Bartoszycha river, leg. I. Dawidziuk no date (BIL). GC02: Supraśl, wet meadow, leg. B. Babczyńska-Sendek 1976 (*). GC11: Ciasne, flooded depression, leg. A. Kawecka 1971 (BIL). GC13: Izbisk, leg. A. Sokołowski 1968 (BIL). GC24: Kobylanka, forest, leg. N. Romańczuk 1973 (BIL). GC30: Dołki, pasture, leg. K. Sokołowska 1970 (BIL). GC33: Cisy, meadow, leg. A. Sokołowski 1968 (BIL); Narew, meadow, leg. A. Sokołowski 1971 (BIL). GC35: Bołtryki, forest, leg. N. Romańczuk 1973 (BIL); valley of rivers Orłówka and Narewka, leg. J. B. Faliński 1960 (BSG); Narew, Siemianówka Reservoir (= Lake Siemianowskie) near railway station Siemianówka, meadow, leg. D. W. Mieńko 1983 (BSG); Narewka river valley, leg. D. Demske 1988 (BSG). GC42: Doratynka, pasture, leg. A. Dackiewicz 1969 (BIL). GC45: Leśna, meadow, leg. J. B. Faliński 1969 (BSG). GC46: Masiewo, wastelands, leg. W. Adamowski 1991 (BSG). GC54: Hajnówka, meadow in forest section 416, leg. A. Sokołowski 1966 (BIL); Skryplewo, wet meadow, leg. R. Waszkiewicz 1973 (BIL); Białowieża, Hajnówka, meadow, leg. W. Adamowski 1992 (BSG). GC55: on Narewka river, forest section 314, leg. A. Sokołowski 1958 (BIL); Browsk, meadow on Hwoźna river, leg. A. Kawecka 1971 (BIL); Zwierzyniec, meadow in forest section 339A, leg. A. Sokołowski 1965 (BIL); Polana Białowieska, meadow, leg. J. B. Faliński 1961, 1963 (BSG); leg. M. Wołosiuk 1978 (BSG); leg. W. Adamowski 1989 (BSG). GC60: Szumki, meadow, leg. N. Romańczuk 1970 (BIL). GC65: Białowieża, meadow on S bank of Narewka river, leg. A. Sokołowski 1956 (BIL); Białowieża, meadow, leg. I. Jacyna 1960 (WA); Polana Białowieska, E of Podolany, meadow, leg. A. Sokołowski 1977 (BIL); Białowieża, Zwierzyniec Forest District forest section 424D, A. Kawecka 1962 (BIL); Białowieża, wet wastelands, leg. B. Pieczerak 1984 (BSG). GC70: Chranibory near Boćki, wet meadow, leg. A. Sokołowski 1968 (BIL). GC73: Starzyna, Leśna, meadow, leg. B. Skiepko 1970 (BSG); Wiluki, meadow, leg. W. Adamowski 1992 (BSG). GC74: Wygon near Hajnówki, meadow, leg. W. Adamowski 1991 (BSG).

Pomorskie. CA38: Jastrzębia Góra, fen in groundwater seepage area near a hotel, leg. H. Piękoś 1964 (KRAM). CA59: Mrzezino, Reda river valley, moist meadow, roadside, leg. M. Jańczak 1971 (UGDA). CA65: Lubowidz, leg. K. Kępczyński 1961 (TRN). CA70: Słupsk, Lasek Południowy (forest), meadow on Słupia river, leg. J. Misiewicz 1971 (SLTC). CA87: 
Kożyczkowo, wet meadow, leg. B. Stachowiak 1982 (UGDA); Garcz, rush bed, leg. B. Stachowiak 1982 (UGDA). CA89: Oliwa Forest, leg. A. Wangerin 1925 (TRN). CA90: $1.5 \mathrm{~km} \mathrm{~S}$ of Zielin, near road to Trzebielin, leg. E. Organiściak 1970 (POZ). CB23: Bytów, edge of Lake Lipusz, leg. W. Galikowska 1960 (POZ); Głuche, edge of Lake Głuche, leg. M. Rejewski 1964 (TRN). CB25: Lake Wdzydze, Sidły Island, meadow, leg. H. Więcław $2008\left({ }^{* *}\right)$. CB33: Gluche, meadow near forest on W edge of Lake Głuche, leg. S. Lisowski 1965 (POZ); Gardlino, wet meadow near Lake Gardliczno Małe, leg. E. Krasicka-Korczyńska 2002 (BYDG). CB43: 1 km N of Józefowo, peatland on W edge of Lake Ostrowite, leg. S. Lisowski, F. Szafrański \& K. Tobolski 1965 (POZ). DB60: Grabowa Góra, SW edge of Lake Udzierz, leg. M. Rejewski 1967 (TRN).

Śląskie. CF39: Taciszów, wet meadow, leg. B. Tokarska 1993 (KTU). CF77: Bolesław, fen, leg. A. Sendek 1964 (KRA). CF79: Marklowice, meadow near shooting range, leg. E. Brzoza 1947 (OLTC). DE50: Kleśniska, alluvial forest, leg. M. Czernicka 1995 (LOD). DE54: Częstochowa Mirów, leg. B. Gajos-Kędzierska 1959 (KRA). DE63: Łobodno-Bartkówka, meadow in Biała Oksza river valley, leg. A. Sendek 1974 (KTU). DE72: Wręczyca Wielka, mine "Malice", riverine thicket, leg. A. Świątczak \& M. Zbroińska 1972 (LOD). DE81: Połamaniec, waterlogged meadows, leg. F. Karo 1877 (WA). DE82: Gać near Blachownia, meadow, leg. J. Hereźniak 1972 (LOD); Aleksandria I, Herby Forest District, fen in groundwater seepage area in river valley, under bridge, leg. J. Hereźniak 1975 (LOD); Blachownia, wet meadow, leg. J. Hereźniak 1988 (LOD). DE83: Częstochowa Błeszno, Długa street, meadow, leg. J. Gajda 1974 (LOD); Częstochowa Błeszno, 32-70 Długa Street, peatland in groundwater seepage area, leg. J. Hereźniak 1987 (LOD); Częstochowa Błeszno, wet meadow, leg. A. Szemet 1998 (LOD). DE85: Małusy Wielkie, moist meadow, leg. A. Sendek 1981 (KTU). DE86: Częstochowa, Zalesice, wet alluvial alder-birch forest, leg. M. Jagiełło 1984 (KRAM). DE87: Koniecpol, nature reserve "Borek", alder carr, leg. R. Lutosiewska \& Z. Rozbicka 1960 (LOD). DE88: Koniecpol-Tomaszów, wet meadow, leg. M. Bielecki 2008 (KRA). DE94: Choroń, waterlogged meadow, leg. W. Duda 1986 (KTU). DF02: E of Kalety, meadow, leg. A. Rostański 2000 (KTU). DF05: Czworaki, wet meadow, leg. K. Jędrzejko \& A. Pelon 2003 (KRAM). DF06: Zdów Młyny, fen in groundwater seepage area, leg. B. Babczyńska-Sendek $1981(*)$; Włodowice, wet meadow, leg. K. Jędrzejko \& A. Pelon 2002 (KRAM); Góra Włodowska, wet meadow, leg. K. Jędrzejko \& A. Pelon 2002 (KRAM). DF07: Pradła, peatland in Krztynia river valley, leg. B. Babczyńska-Sendek 1980 (*). DF12: Miasteczko Śląskie, waterlogged meadow W of smelter, leg. K. Rostański 1975 (KTU). DF14: Winowno, meadow in stream valley, leg. A. Sendek 1978 (KTU); Las Brudzowice (forest), wet meadow, leg. M. Krzyżek \& K. Jędrzejko 1999 (KRAM). DF19: Żarnowiec near Pilica, mire, leg. B. Chwastowski 1978 (KRAM). DF25: Tucznawa, wet meadow, leg. K. Jędrzejko 1974 (KTU); Poręba, Las Porębski (forest), moist forest meadow, leg. A. Rok \& M. Sochacka 1998 (SZUB); Poręba near Zawiercie, poor fen, leg. A. Błońska 2009 (*). DF28: Wierbka, moist meadows in Pilica river valley near Sławniów-Baran, leg. B. Babczyńska-Sendek $1980\left(^{*}\right)$; Wierbka, close to border with Sławniów, emergent vegetation, leg. B. Babczyńska-Sendek 1980 (*). DF31: Zabrze Maciejów, leg. A. Sendek 1972 (KTU). DF33: Będzin Pustkowie, meadow, leg. A. Sendek 1978 (KTU). DF34: Dąbrowa Górnicza-Zielona, moist meadow, leg. A. Sendek 1975 (KTU); Dą̧rowa Górnicza, meadow, leg. B. Babczyńska-Sendek 1988 (*). DF35: Niegowonice, ditch, leg. M. Kucia 1987 (KTU); S of Niegowonice, meadow, leg. B. Babczyńska-Sendek 1988 (*); Dąbrowa Górnicza-Przymiarki, wet meadow, leg. T. Nowak 1992 (KTU); Sławków Zagródki, meadow, leg. G. Kłys 2000 (KTU). DF36: near Biała Przemsza river, Błędów Desert, leg. R. Kobendza 1925 (WA). DF41: Gliwice, leg. C. Dziatzko 1902 (OPOL). DF43: Katowice Muchowiec, leg. A. Rostański 1990 (KTU). DF45: Jaworzno-Dobra-Wilkoszyn, forest meadow, leg. A. Rostański 1993 (KTU). DF84: Bujaków, wetland, 480 m a.s.l., leg. B. Kotońska 1980 (KRA). DF91: Skoczów, fen in groundwater seepage area in N part of town, leg. S. Pelc 1963 (KRA); Górki Wielkie, wet meadow, leg. J. Maciejczek 1990 (KRA). DF92: Jasionki near Jaworze, leg. Zapałowicz 1904 (KRAM). DF94: Luboń Wielki, moist meadow in coniferous forest close to Rabka, leg. B. Kotońska 1966 (KRA); Kozy Gaje, fen in groundwater seepage area, leg. B. Kotońska 1980 (KRA). DF95: Kocierz Mierzczaki, fen in groundwater seepage area, leg. B. Kotońska 1986 (KRA); Kocierz Basie, fen in groundwater seepage area, leg. B. Kotońska, 1986 (KRA). DG01: Cisownica-Wendoły, S part, fen in groundwater seepage area, leg. S. Pelc 1962 (KRA); N slope of Mała Czantoria, fen in groundwater seepage area, leg. S. Pelc 1962 (KRA). DG04: Czernichów, wet meadow, leg. B. Kotońska 1980 (KRA). DG05: Gachowizna (mount), N slope, fen in groundwater seepage area, 700 m a.s.l., leg. K. Szczerbińska 1965 (KRAM). DG12: Cieszyn, Czarna Wisełka stream valley, leg. K. Rostański 1973 (KTU); Istebna, nature reserve "Wisła", Biała Wisełka stream valley, leg. K. Jędrzejko, H. Klama \& J. Żarnowiec no date (KRA); Przysłop, fen in groundwater seepage area on right bank of Czarna Wisełka, leg. W. Buława 1968 (SZUB). DG14: Grojec (hill) near Żywiec, grassland with rock outcrops, $360 \mathrm{~m}$ a.s.l., leg. K. Nowak 1986 (KTU); Grojec near Żywiec, wet meadow, 450 m a.s.l., leg. K. Nowak 1985 (KRAM); Radziechowy, wet meadow, leg. B. Fojcik 1987 (KTU); Komarnik, tall herb communities along stream, leg. M. Wybczak 2001 (KTU). DG22: Andziołówka, wet meadow, leg. M. Dudzik 1971 (LOD); Olecki Górne, wet meadow, leg. M. Dudzik 1972 (LOD); Szymcze, wet meadow, leg. M. Dudzik 1972 (LOD). DG23: Barania Góra, Kamesznica, leg. A. Żmuda 1914 (KRAM). DG24: Romanka, Jeleśnieńska Młaka (fen), leg. K. Szczerbińska 1965 (KRAM); Romanka, source of Tokarka stream, left tributary of Sopotnia Wielka stream, 1050 m a.s.l., leg. K. Szczerbińska 1965 (KRAM); Marszałkowska Młaka (fen), leg. K. Szczerbińska 1965 (KRAM); valley of Sopotnia Wielka, moist meadow, 650 m a.s.l., leg. K. Szczerbińska 1965 (KRAM); valley of Bystra stream (Złotna), fen in groundwater seepage area, 700 m a.s.l., leg. K. Szczerbińska 1965 (KRAM); Lipowska, Hala Rysianka, fen in groundwater seepage area, 1250 m a.s.l., leg. K. Szczerbińska 1965 (KRAM). DG25: Pilsko, "na Cebuli", wet peaty meadow, 1330 m a.s.l., leg. Seńkowski 1938 (KRA); Hala Cudzichowa, fen in groundwater seepage area, $1180 \mathrm{~m}$ a.s.l., leg. K. Białecka 1970 (KRA); Pilsko, Hala Miziowa, 1290 m a.s.l, fen in groundwater seepage area, leg. K. Białecka 1970 (KRA); 
Pilsko (mount), leg. K. Jędrzejko, H. Klama \& J. Żarnowiec 1984 (KRA). DG33: Muńczoł, springhead leg. B. Kowalczyk 1993 (KTU). DG34: W of Soblówka, forest roadside, 650 m a.s.1., leg. J. Koopman 2009 (*).

Świętokrzyskie. DE69: Kluczewsko, peaty meadow, leg. J. Błaszczyk 1949 (KRA); Jakubowice, wet meadow, leg. M. Bielecki 2009 (KRA). DE88: Gabrielów, wet meadow, leg. M. Bielecki 2008 (KRA). DE89: Kąty, wet meadow, leg. M. Bielecki 2009 (KRA). DE99: Bieganów, waterlogged willow thicket, leg. M. Bielecki 2009 (KRA). DF09: Obiechów, alder wood edge, leg. J. Błaszczyk 1949 (KRA); Chałupki near Szczekociny, waterlogged willow thicket, leg. M. Bielecki 2009 (KRA). EE32: Trzemoszna, nature reserve "Białe Ługi", meadow, leg. M. Wayda 1983 (KRA); nature reserve "Białe Ługi", forest mire, leg. M. Wayda 1984 (KRA). EE33: Garb Gielniowski, poor fen, leg. M. Podgórska 2005 (KRA); Dwa Jaja near Końskie, waterlogged site in forest, leg. M. Podgórska 2004 (KRA). EE42: Niebo, moist forest path, leg. A. Trojecka-Brzezińska 2010 (KRA). EE45: Dalejów, moist glade, leg. H. Błaszczyk 1939 (KRA); Podgórki, ditch near railway tracks, leg. M. Podgórska 2005 (KRA); Bliżyn, wet meadow, leg. H. Piękoś 1969 (KRAM); Świnia Góra near Bliżyn, leg. H. Piękoś 1964 (KRAM); Bliżyn near Kielce, wet meadow, leg. H. Piękoś 1966 (KRAM). EE46: S of village Kierz Niedźwiedzi near Szydłowiec, fen near river, leg. M. Nobis 2003 (KRA). EE47: E of Trąbowiec, meadow in Iłżanka river valley, leg. M. Nobis 2002 (KRA). EE51: Radoszyce, moist meadow, leg. A. Trojecka-Brzezińska 2010 (KRA); between Grodzisko and Sachalinka, emergent vegetation, leg. A. Trojecka-Brzezińska 2010 (KRA). EE52: Plenna, wet meadow, leg. A. Trojecka-Brzezińska 2010 (KRA); Radoska, wet meadow, leg. A. Trojecka-Brzezińska 2010 (KRA). EE53: N of Grębosze, wet meadow, leg. A. Trojecka-Brzezińska 2010 (KRA). EE55: Suchedniów near Kielce, wet meadow, leg. A. Jasiewicz 1964 (KRAM). EE57: $1 \mathrm{~km}$ N of W end of Michałów Wiejski, fen, leg. M. Nobis 2003 (KRA); Starachowice, moist sites on a spoil tip, leg. M. Nobis 2003 (KRA). EE58: Działki near Brody Iłżeckie, peatland edge, leg. M. Nobis 2002 (KRA); Przymiarki, meadow, leg. M. Nobis 2002 (KRA). EE63: Mokry Bór, Czarna Woda river valley, peatland, leg. J. Jakubowska-Gabara 1976 (LOD). EE64: Kielce-Kostomłoty, fen, leg. I. Kulej \& B. Maciejczak 1986 (KTC); Kielce-Wiśniówka Duża, leg. J. Michta \& B. Maciejczak 1988 (KTC). EE65: Ciekoty, leg. Głowacka \& Lubańska 1961 (TRN). EE66: Grabowo near Bukowa Góra, wet meadow, leg. L. Fagasiewicz 1963 (LOD); between Psary and Bukowa Góra, meadow, leg. L. Fagasiewicz 1963 (LOD). EE69: 1 km SW of Janiki, fen, leg. R. Piwowarczyk 2003 (KRA); Ostrowiec Świętokrzyski, woodland NW of town, peaty meadow, leg. R. Piwowarczyk 2003 (KRA). EE70: Kotowie, moist deciduous forest, leg. M. Bielecki 2008 (KRA); Czerwonka, S of Ostrowiec, alder wood, leg. M. Bielecki 2007 (KRA). EE72: N of Lesica, moist meadow, leg. G. Łazarski 2013 (KRA). EE73: Chęciny, Bobrzyczka river valley, leg. B. Maciejczak 1999 (KTC); N of E end of village Szewce, fen in groundwater seepage area, leg. G. Łazarski 2013 (KRA). EE74: Góra Dymińska (hill), forest glade, leg. A. Chatys \& E. Bróż 1987 (KTC). EE75: Kielce, leg. K. Zazula \& B. Maciejczak 1975 (KTC); Cedzyna, wet meadow, leg. E. Bróż \& Z. Bednarowska 1985 (KTC); Grupa Otroczna, moist forest glade, leg. E. Bróż \& M. Chrzanowska 1987 (KTC); Kielce, Cedro-Mazur, leg. E. Bróż \& Z. Bednarowska 1985 (KTC). EE77: Rudka, Góra Chełmowa (hill), on Pokrzywianka river, leg. J. Jakubowska-Gabara 1976 (LOD). EE80: Ludwinów-Huby, leg. M. Bielecki 2009 (KRA). EE81: Kielce, meadow, leg. J. Stępniewska \& B. Maciejczak 1987 (KTC). EE82: between Bizorenda and Szczepanów, moist meadow, leg. B. Piwowarski 2010 (KRA); Bizorenda, emergent vegetation, leg. B. Piwowarski 2011 (KRA); ); E end of village Stawki near Polichno, moist meadow, leg. G. Łazarski 2013 (KRA). EE83: Sitkówka, moist meadow, leg. G. Łazarski 2013 (KRA); S of W end of village Brzezina near Morawica, moist meadow, leg. G. Lazarski 2013 (KRA); N of E end of village Radkowice near Chęciny, peaty ditch, leg. G. Łazarski 2013 (KRA); W of village Lipowice near Chęciny, moist, peaty meadow, leg. G. Łazarski 2013 (KRA). EE84: Dyminy-Bilcza, leg. K. Kaznowski 1925 (KRAM). EE85: E of Daleszyce, moist meadow, leg. W. Paul 1988 (KRAM); Słopiec, about 20 km SE of Kielce, peatland, 250 m a.s.l., leg. J. Wójcicki 1973 (KRAM); Czerwona Woda, N of Niwki Daleszyckie, fen, leg. W. Paul 1990 (KRAM). EE86: E of Makoszyn, moist thicket, leg. W. Paul 1990 (KRAM). EE90: Ślęcin, moist meadow, leg. B. Piwowarski 2008 (KRA). EE91: Zdanowice, moist meadow, leg. B. Piwowarski 2008 (KRA); NE of Choczewy Las Choczewy (forest), wet coniferous forest, leg. B. Piwowarski 2008 (KRA); Zdanowice, fen in groundwater seepage area, leg. B. Piwowarski 2009 (KRA). EE92: Mzurowa, wet coniferous forest, leg. B. Piwowarski 2008 (KRA); Jędrzejów-Wilanów, drained forest mire, leg. B. Piwowarski 2011 (KRA). EE98: Zamajdanie, meadow, leg. I. Witkowska \& A. Przemyski 1990 (KTC). EF01: Warzyn, moist meadow, leg. B. Piwowarski 2009 (KRA). EF03: Imielno, ponds, moist meadow, leg. B. Piwowarski 2006 (KRA); Niebyła, moist meadow, leg. B. Piwowarski 2011 (KRA). EF07: Kurozwęki, partly overgrown pond, leg. M. Wayda 1983 (KRA); SW of Wymysłów, moist meadow, leg. M. Nobis 2003 (KRA). EF08: Staszów, moist meadow, leg. J. Błaszczyk 1950 (KRA). EF09: Wola Wiśniowska, leg. unknown 1973 (KRA). EF14: S of Włochy, meadow, leg.

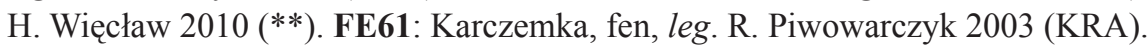

Warmińsko-Mazurskie. DB55: Susz, meadow near Lake Gaudy, leg. H. Wojciechowska \& B. Polakowski 1955 (OLTC). DB59: Warlity Wielkie, drained bottom of former fish pond, leg. J. Steckiewicz 1938 (OLTC). DB69: Brzydowo, wet meadow, leg. R. Banaś \& W. Pietraszewski 1983 (OLTC). DB76: Iława, nature reserve "Karaś", peatland near E part of lake, leg. H. Wojciechowska \& B. Polakowski 1954 (OLTC). DB79: Ostróda, Francuskie Lake, meadow, leg. J. Hutorowicz 1955 (TRN). EB12: Łaniewo, Potar Lake, wet meadow, leg. M. Dzwończak 1979 (LOD). EB15: Sękity, meadow, leg. T. Szarejko 1971 (OLTC). EB24: Pierwagi, moist meadow, leg. L. Olesiński 1968 (OLTC). EB27: Łężany, Samławki pond, leg. M. Środa 2003 (OLTC). EB45: Rzeck, meadow on drained peatland, leg. L. Olesiński 1967 (OLTC); Pudlag, poor fen, leg. U. Boińska 1970 (TRN). EB46: Dymer, moist meadow, leg. J. Hutorowicz 1954 (OLTC), Dymer, leg. L. Olesiński 1956 (OLTC); Dymer, leg. M. Olkowski 1956 (OLTC); Kobułty, wet meadow, leg. L. Olesiński 1967 (OLTC); Parleza Wielka, meadow near road 
Biskupiec-Mragowo, leg. T. Szarejko 1981 (OLTC). EB49: S of Lipowo, meadow, leg. W. Pietraszewski 1976 (OLTC); Faszcze, meadow, leg. W. Pietraszewski 1978 (OLTC); Strzałowo, clearing behind meadows, leg. T. Traczyk 1976 (KRAM); Strzałowo, fen, leg. T. Traczyk 1978 (KRAM). EB52: Olsztyn Posorty, peaty meadows, leg. H. Wojciechowska 1953 (OLTC); Nowa Ukta, fen, leg. A. Hudaszek 1980 (KRA). EB56: Popowa Wola, moist meadow on drained peatland, leg. L. Olesiński 1967 (OLTC); Targowska Wólka, meadow on peatland, leg. T. Szarejko 1981 (OLTC). EB64: Tylkówko, edge of fish pond, leg. M. Środa 1998, 1999 (OLTC); Tylkówko, waterlogged meadow, E edge of fish pond, leg. M. Środa 1999 (OLTC). EB65: Pasym, Leleszki complex of fish ponds, leg. M. Środa 2001 (OLTC). EB75: Szczytno, peatland near lake, leg. W. Rafflewska 1977 (TRN). EB90: SE of Dąbrówno, meadow, leg. H. Więcław 2010 (**). FA85: Hajnówek, Rominta Forest (Puszcza Romincka), forest roadside, leg. A. Tyc 2005 (KRA). FA87: N of Kramnik, fen, leg. H. Więcław 2010 (**). FB03: Lipowo, moist clearing, leg. B. Polakowski 1956 (OLTC). FB10: Giżycko, moist urban forest, leg. A. Łubaszewska 1956 (LOD). FB16: Szczecinki, meadow, leg. J. Żurawski 1969 (BIL). FB22: Szczepanki, nature reserve "Łąki Staświńskie", leg. T. Szarejko 1982 (OLTC). FB27: Wierzbowo, meadow, leg. A. Kawecka 1970 (BIL). FB31: E of Borki, nature reserve "Nietlickie Bagno", meadow, leg. H. Więcław $2010(* *)$. FB33: Stare Juchy, lake edge, leg. M. Strugała 1971 (BIL). FB34: Bałamutowo, wet meadow, leg. M. Strugała 1971 (BIL). FB35: Stradun, mire, leg. D. Myszkowska 1981 (WA). FB40: Mikołajki, wet meadow near Lake Mikołajewskie, leg. J. Hutorowicz 1957 (OLTC); Woźnice, meadow at N edge of Lake Łuknajno, leg. W. Pietraszewski \& T. Szarejko 1979 (OLTC). FB46: Kucze, Lake Krzywe, mire, leg. G. Przybysz 1981 (WA). FB52: Lysonie, meadow, leg. T. Szarejko 1973 (OLTC). FB53: Drygały, peatland near Pogorzel, leg. J. Borowski 1977 (TRN).

Wielkopolskie. BC72: Sieraków, Lake Kłosowskie, leg. M. Jasnowski 1957 (SZCZ). BC97: Lusówko, moist alder thicket, leg. W. Staniewska 1962 (BYDG). BC99: Dziewicza Góra (hill) near Poznań, meadows and gullies SE of forester's lodge. leg. W. Olkiewicz 1934 (POZ). BD18: Ludwikowo, S edge of Lake Góreckie, leg. E. Goślinowska 1927 (POZ). BD59: Gostyń, meadow near Obra river, leg. Bojarska 1964 (POZ), leg. J. Gumienna 1964 (POZ). BD69: Podrzecze, meadow, leg. I. Wujczak 1964 (PUMA), (KRAB). CC31: Smogulec, moist meadow, leg. A. Bosacki 1975 (TRN). CC50: Wagrowiec, meadow, leg. M. Szostak 1976 (POZ). CC70: Sława, meadow near Lake Włókno, leg. F. Krawiec 1928 (POZ). CD81: Baszków, meadow, leg. B. Kotewicz 1965 (WRSL). CE14: W of Lipnik, meadow, leg. E. Gola 1990 (WRSL). DD11: Szatanowo, mire, leg. L. Kucharski 1983 (LOD).

Zachodniopomorskie: AB56: E of Ogorzele, forest meadow, leg. H. Więcław 2010 (**). AB62: Nowa Jasienica, leg. M. Jasnowski 1960 (SZCZ); NW of Zalesie, nature reserve "Świdwie", meadow, leg. H. Więcław 2010 (**). AB64: Krępsko, ecological area "Bagna Krępskie", meadow, leg. H. Więcław 2008 (**); Modrzewie, ecological area "Bagna Krępskie", meadow, leg. H. Więcław 2008 (**); Budzeń near Stepnica, meadow, leg. B. Kurnicki 2012 (SZUB). AB72: Pilichowo I, fen, leg. M. Jasnowski 1962 (SZCZ). AB78: E of Chociwel, moist meadow, leg. H. Więcław 2000 (**). AB88: Dzwonowo, meadow, leg. M. Jasnowski 1968 (SZCZ). AB95: Kołbacz, meadows near Płonia river in direction of Jezierzyce, leg. F. Celiński 1962 (POZ). AC02: Krajnik, moist meadow on Odra river, leg. M. Wilhelm 2009 (SZUB). AC24: Lake Sitno Wielkie, peatland edge near W end of lake, leg. J. Izydorek 1977 (SLTC). AC31: Piasek, edge of waterhole in forest, leg. E. Stępień 1999 (SZUB). AC41: Brochucin, Natural Landscape Complex "Dolina Słubi", alluvial forest, leg. E. Stępień \& M. Myśliwy 1998 (SZUB). AC47: Moczydło, edge of Lake Listek, leg. M. Myśliwy 1999 (SZUB); forest section 557b, Barlinek Forest District, meadow, leg. M. Myśliwy 1999 (SZUB). AC48: Lipy, planned nature reserve "Lilie Wodne", peatland, leg. M. Myśliwy 2000 (SZUB). BB01: N of Kukinia, ditch in peatland, leg. B. Bosiacka 2003 (SZUB). BB08: Wielin, meadow, leg. J. Koopman 2009 (*). BB18: Żydowo, meadow, leg. K. Stepczyńska \& M. Korczyński 1985 (BYDG). BB35: planned nature reserve "Cisy Tychowskie”, alder carr, leg. Grabowska 1976 (TRN). BB43: Połczyn Zdrój, leg. J. Wiśniewski 1972 (WA). BB44: Buślarki, meadow near a tributary of Parsęta river, leg. M. Myśliwy \& E. Stępień 2001 (SZUB). BB61: Zagórzyce, moist meadow, $\mathrm{W}$ end of village, leg. M. Wilhelm 2011 (SZUB); Zagórzyce, emergent vegetation, W end of village, leg. M. Wilhelm 2011 (SZUB). BB64: Kluczewo, W edge of Lake Prosino, alder wood, leg. B. Bosiacka 2003 (SZUB); Kluczewo, alder carr and meadow near W edge of Lake Prosino, leg. H. Więcław 2009 (**). BC00: Kiełpino near Choszczno, forest meadow and alder carr, leg. H. Więcław 2011 (**). BC13: Drawno, nature reserve "Drawa”, sedge bed, leg. M. Jasnowski 1974 (SZCZ).

\section{Carex hostiana DC.}

Dolnośląskie. BE12: Gorzyca near Lubin, wet meadow, leg. Z. Kącki \& Z. Dajdok 2002 (WRSL). BF06: Grzępa, leg. R. Schultz 1892 (TRN).

Lubelskie. GE35: peatland reserve "Brzeźno" near Chełm Lubelski, leg. B. Chwastowski 1978 (KRAM). GE82: Zamość, peaty meadows, leg. F. Berdau no date (WA).

Małopolskie. EF51: Czernichów near Kraków, meadow in Vistula river valley, leg. K. Zarzycki 1956 (KRAM). EF72: Grodkowice, Niepołomice Forest, moist ditch, leg. A. Jasiewicz 1951 (KRAM).

Mazowieckie. FD07: Kamianki Lackie, fen in Kołodziejka river valley, leg. Z. Głowacki 1977 (SLTC).

Pomorskie. CA36: Dębki, nature reserve "Piaśnickie Łąki", meadow, leg. H. Więcław 2009 (**). CA37: 1 km E of Dębki, moist meadow, leg. L. Kowalska 1982 (UGDA).

Śląskie. DF02: Psary, N slope of Grojec (hill) waterlogged meadow at base of limestone hill, leg. J. Hereźniak 1978 (LOD). DF25:, between Zawiercie and Poręba Mrzygłodzka, meadow in forest, leg. K. Kaznowski 1919 (POZ). 
Świętokrzyskie. EE74: Domaszowice near Kielce, meadow, leg. K. Kaznowski 1928 (WA). EE82: S of E end of village Stawki near Polichno, moist meadow, leg. G. Łazarski 2013 (KRA). EE83: S of W end of village Brzezina near Morawica, moist meadow, leg. G. Łazarski 2013 (KRA). EF14: S of Włochy near Pińczów, meadow, leg. H. Więcław 2010 (**). EF33: Sielec Biskupi near Skalbmierz, waterlogged depressions in former gypsum quarry, leg. J. Nęcka \& Z. Sztyler 1974 (KRAM). Warmińsko-mazurskie. EB69: Pisz Forest (Puszcza Piska), fen near a lake, leg. A. \& J. Kornaś 1952 (KRA).

\section{Carex lepidocarpa Tausch}

Dolnośląskie. BE27: SE of Radecz, fen, leg. Z. Głowacki 1972, 1973 (WSRP).

Kujawsko-Pomorskie. CB78: Wałkowiska, fen near E edge of Lake Sierosławek, leg. H. Więcław 2009 (**). CC31: Folusz, meadow, leg. E. Krasicka-Korczyńska 2005 (BYDG). DC10: Papowo Biskupie, moist meadow, leg. Radtke 1981 (TRN). DC12: Ludowice, Żgniłka mire, leg. K. Stempska 1961 (POZ).

Lubelskie. FE07: Wólka Krasienińska, swamp in forest, leg. A. Łuczycka 1969 (LBLM). FE28: Sobianowice, meadows near Bystrzyca river, leg. D. Fijałkowski 1951 (LBLM); Spiczyn, meadows near Bystrzyca river, leg. D. Fijałkowski 1951 (LBLM). FE37: Zemborzyce, meadows near Bystrzyca river, leg. D. Fijałkowski 1951 (OLTC). FF08: Dąbrowica, peatland on sandy slope, leg. D. Fijałkowski 1964 (OLTC). GE00: Rozkopaczów, meadows along Tyśmienica river, leg. D. Fijałkowski 1960 (LBLM). GE02: Wólka Wytycka, peatland near Lake Długie, leg. L. Fagasiewicz 1964 (LOD); Wólka Wytycka, nature reserve "Krowie Bagno", leg. M. Szewczyk 1978 (KRAM), leg. B. Chwastowski 1978 (KRAM). GE11: Ciesacin near Garbatówka, wet meadow, leg. J. Zalewska-Gałosz 2005 (KRA). GE13: Tarnów near Urszulin, meadow, leg. D. Fijałkowski 1965 (LBLM). GE35: Chełm Lubelski, nature reserve "Brzeźno", leg. B. Chwastowski 1978 (KRAM). GE45: Wolawce near Chełm Lubelski, calcareous fen, leg. A. Jasiewicz 1964 (KRAM), leg. A. Sokołowski 1964 (BIL), leg. J. Serwatka 1964 (SZUB). GE81: S of Szczebrzeszyn, moist meadows near Wieprz river, leg. A. Jasiewicz 1967 (KRAM). GE90: Zwierzyniec, meadows near Wieprz river, leg. D. Fijałkowski 1962 (LBLM); Żurawnica, meadows near Wieprz river, leg. D. Fijałkowski 1964 (OLTC). GF01: Górecko Kościelne, poor fen on Szum river, leg. D. Fijałkowski 1965 (KRA). GF11: Józefów, moist meadow, leg. A. Sokołowski 1965 (BIL).

Lubuskie. AC46: Chłopiny, leg. M. Jasnowski 1959 (SZCZ); Chłopiny, N of Gorzów Wielkopolski, fen in groundwater seepage area, leg. J. Koopman 2008 (*). AC67: Gorzów Wielkopolski, waterlogged pasture near Teatralna Street, leg. J. Misiewicz 1968 (SZCZ). AC76: Kołczyn, mire, leg. I. Lis 1992 (SZUB). AD09: Nowy Dworek, edge of Lake Paklicko Wielkie, leg. W. Żukowski 1966 (POZ). AD18: Borów, drainage ditch between meadows near Lake Wilkowskie, leg. Z. Głowacki 1968 (WRSL). BD63: Lgiń, meadows near S edge of Lake Lgińsko, leg. Cz. Brzozowski 1995 (POZ).

Małopolskie. DF27: Bydlin-Tarnówka, fen at NW end of village, leg. B. Babczyńska-Sendek 1980 (*). DF36: Bolesław in direction of Błędów Desert, meadow ditch, leg. J. Dobrzańska 1945 (KRA); Laski near Olkusz, Biała river valley, fen in groundwater seepage area, leg. H. Trzcińska-Tacik 1989 (KRA); Błędów Desert, fen, leg. A. Jasiewicz 1951 (KRAM). DF37: Cieślin, peatland behind Dom Ludowy (community centre) leg. B. Babczyńska-Sendek 1980 (*); Cieślin-Pustki, poor fen on Sączenica river, leg. B. Babczyńska-Sendek $1981\left(^{*}\right)$. DF38: W of Wolbrom, meadow, leg. B. Babczyńska-Sendek $1981\left(^{*}\right)$; Wolbrom, W of town, peatland between road and railway tracks, leg. B. Babczyńska-Sendek 1981 (*). DF55: Luszowice, waterlogged meadow in Luszówka valley, leg. I. Mazaraki 1967 (KRAM); Luszowice, fen in groundwater seepage area near railway tracks, leg. I. Mazaraki 1967 (KRAM); Chrzanów, waterlogged meadow, leg. L. Olesiński 1973 (KRAM). DF58: Dolina Sąspowska, wet meadow, leg. unknown 1965 (KRAM). EF32: Słupów, ditches and moist meadows in Nidzica river valley, leg. W Bartoszek 2005 (KRA). EF72: Grodkowice near Niepołomice, mire, leg. Łańcucka 1938 (KRA).

Mazowieckie. ED02: Nowiny, alder forest, leg. K. Nowak 1971 (WA). ED16: Warsaw, Bemowo Forest, moist meadow, leg. I. Kirpluk 1986 (WA). ED19: Feliksów, fen, leg. Z. Głowacki 1990 (WSRP). EE37: about 2 km W of Pakosław near Iłża, peaty meadow, leg. M. Nobis 2003 (KRA), leg. M. Sychowa 1954 (KRAM), leg. A. Jasiewicz 1954 (KRAM), leg. W. Miś 1954 (KRAM), leg. T. Tacik 1954 (KRAM). EE38: Pakosław near Ilża, peaty meadow, about $1.5 \mathrm{~km} \mathrm{~W}$ of village, leg. M. Nobis 2002 (KRA).

Podkarpackie. FG24: Wernejówka, eutrophic fen in groundwater seepage area, leg. K. Oklejewicz 2004 (OLTC); Wernejówka, eutrophic meadow, $460 \mathrm{~m}$ a.s.l., leg. G. Voncina 2004 (KRA).

Podlaskie. FA88: Smolniki, mire, leg. A. Sokołowski 1980 (BIL); Kleszczówek, mire, leg. A. Sokołowski 1969 (BIL). FA97: Szeszupka near Jeleniewo, springhead, leg. A. Sokołowski 1975 (BIL); Bachanowo, fen in groundwater seepage area, leg. A. Kawecka 1981 (BIL); Turtul, meadow, leg. A. Kawecka 1981 (BIL). FB06: Filipów Pierwszy, moist meadow near waterlogged thicket in Rospuda river valley, leg. A. Pliszko 2008 (KRA); Filipów Pierwszy, fen in Rospuda river valley, leg. A. Pliszko 2009 (KRA). FB08: Osowa, fen in Czarna Hańcza river valley, leg. A. Pliszko 2010 (KRA). FB17: Kamionka Stara, fen "Bagno Parchacz", leg. A. Pliszko 2009 (KRA). FB19: Gawrych-Ruda, peatland at W edge of Lake Okragłe, leg. A. Sokołowski 1964 (BIL); Rosochaty Róg, poor fen, leg. J. Trzeciak 2000 (LOD). FB28: Szczebra, Rospuda river valley, peatland near viewing point W of village, leg. H. Więcław 2010 (**). FC98: Zajęczniki E, leg. P. Kalinowski 2013 (*). GB00: Murowany Most, meadow, leg. S. Fieńko 1969 (BIL); Remieńkiń, peaty meadow S of Lake Żubrowo, leg. A. Kawecka 1969 (BIL); Żubrówka, poor fen, leg. A. Kawecka 1969 (BIL). GB01: Berżniki, peatland at E edge of Lake Gajlik, leg. E. Jabłońska 2001 (WA); forest at NW edge of Lake Gajlik, leg. E. Jabłońska 2001 (WA). GB02: Berżniki, meadow in village, 
near Kunisjanka stream, leg. H. Więcław 2010 (**). GB10: Sarnetka near Suwałki, fen in groundwater seepage area, leg. A. Sokołowski 1988 (BIL). GB11: Giby, moist meadow, leg. B. Sudnik 1975 (WA); Giby, bog surrounded by fields, near road Giby-Sejny, leg. T. Szarejko 1987 (OLTC). GB40: Jastrzębna near Augustów, Biebrza river catchment area, leg. M. Jasnowski 1954 (SZCZ); Krasnybór near Augustów, Biebrza river catchment area, leg. M. Jasnowski 1954 (SZCZ). GB82: Sokółka, fen, leg. Z. Głowacki 1992 (WSRP).

Pomorskie. CA58: Nanice, meadow ditch, leg. R. Markowski 1968 (UGDA). CA59: Osłonino, low-sedge fen in nature reserve "Beka", S of village, leg. H. Więcław 2009 (**). CA70: Słupsk, pond edge and meadow, leg. J. Smyl 1979 (SLTC). CA86: Miechucino, narrow pass between Lakes Wielkie and Długie, meadow, leg. M. Jąkalska 1982 (UGDA). CB22: 5 km SW of Lipnica, fen near settlement Klewiska, leg. S. Lisowski, F. Szafrański \& K. Tobolski 1968 (POZ). CB23: Luboń, peatland near Lake Kielskie, leg. S. Lisowski 1965 (POZ). CB25: Schodno, SW edge of Lake Bielawy, meadow and poor fen, leg. H. Więcław 2008 (**); Lake Wdzydze, Sidły Island, meadow, leg. H. Więcław 2008 (**). CB26: S of road Wdzydze Kiszewskie-Gołuń, ecological area "Kiszewskie Bagno" at N edge of Lake Gołuń, poor fen, leg. H. Więcław 2008 (**). CB33: Asmus, peatland near Lake Stawek, leg. S. Lisowski, F. Szafrański \& K. Tobolski 1964 (KRA, POZ). CB43: Chojnice, peaty edge of Lake Płęsno, leg. W. Gugnacka 1974 (KTU, LOD, POZ, TRN).

Śląskie. DE85: Małusy Wielkie, moist meadow, leg. A. Sendek 1981 (KTU). DF02: Psary, N slope of Grojec (hill), moist meadow at base of limestone hill, leg. J. Hereźniak 1978 (LOD). DF08: Ołudza Nowa, fen at NW end of village, leg. B. Babczyńska-Sendek 1981 (*). DF19: Żarnowiec near Pilica, mire, leg. B. Chwastowski 1978 (KRAM). DF26: Hutki-Kanki, meadow in Centuria river valley, leg. B. Babczyńska-Sendek 1981 (*). DF34: Strzemieszyce, near road to Kazimierz, alder carr, leg. A. Sendek 1973 (KTU); Sosnowiec Zagórze, fen in groundwater seepage area, on a meadow near mine Porąbka, leg. A. Sendek 1977 (KTU); Dąbrowa Górnicza, Piekło, moist meadows near Lake Pogoria II, leg. A. Sendek 1982 (KTU); Dąbrowa Górnicza, Lake Pogoria I, fen in groundwater seepage area, leg. A. Czylok 1995 (KTU); Dąbrowa Górnicza, Antoniów, poor fen, leg. A. Błońska 2011 (*). DF44: Sosnowiec Bory, anthropogenic wetlands in old sandpit, leg. A. Błońska 2011 (*). DF45: Groniec, N of village, waterlogged site in forest, leg. J. Drobnik 2000 (KTU). DF92: Jasionki near Jaworze, leg. Zapałowicz 1904 (KRAM).

Świętokrzyskie. EE81: Lasochów (Dziadówki), silty edge of a water body, leg. M. Bielecki 2010 (KRA). EE92: Ignacówka, ditch in farmland, leg. B. Piwowarski 2010 (KRA). EF13: Bełk, fen in groundwater seepage area, leg. B. Piwowarski 2011 (KRA). EF15: Mlyny, wet meadow, leg. H. Piękoś \& A. Jasiewicz 1964 (KRAM).

Warmińsko-mazurskie. FB61: Ruciane, Roś fen, leg. J. Staszkiewicz 1952 (KRAM).

Wielkopolskie: BC94: Zgierzynka, meadow, leg. W. Żukowski 1975 (POZ). BC97: Lusowo, leg. J. Grajek, 1957 (LOD). BC99: Dziewicza Góra (hill) near Poznań, wet meadows E of Miękowo, leg. W. Alkiewicz 1965 (POZ). BD08: Poznań Wola, waterlogged meadow near Lake Rusałka, leg. S. Lisowski 1955 (POZ). BD18: Ludwikowo, S edge of Lake Góreckie, leg. E. Goślinowska 1927 (POZ). BD58: Stary Gostyń, partly overgrown peaty pool, leg. O. Kaczmarek 1939 (POZ); Stary Gostyń, mire, leg. K. Latowski \& W. Żukowski 1975 (POZ). CC70: Sława, meadow near Lake Włókno, leg. F. Krawiec 1928 (POZ). CC84: 1 km W of Rudki, peatland N of railway tracks, leg. Z. Mrugowska 1970 (POZ). CC90: Promno, wet meadow near Lake Dębiec, leg. F. Krawiec 1929 (POZ); Promno, sedge fen near railway station, leg. A. Danowska 1932 (POZ). CD09: Sycewo, wet meadow, leg. Z. Mikołajczyk 1964 (LOD).

Zachodniopomorskie: AB23: W of Kodrąbki, forest meadow, leg. H. Piotrowska 1960 (UGDA). AB24: NW of Kołczewo, meadow, leg. H. Piotrowska 1966 (UGDA). AB64: Modrzewie, ecological area "Bagna Krępskie", meadow, leg. H. Więcław 2008 (**). AB97: Tychowo, moist meadow, leg. W. Bacieczko 1980 (SZCZ). AC05: Grędziec-Wierzbno, edge of Lake Miedwie, leg. M. Jasnowski 1971 (SZCZ); Będgoszcz, waterlogged meadow at N edge of Lake Będgoszcz, leg. H. Więcław 2010 (**). AC16: Lubiatowo, N edge of Lake Torfowe, leg. H. Więcław 2011 (**); Zaborsko, meadow, SE edge of Lake Zaborsko, leg. H. Więcław 2011 (**). AC25: Sitno, nature reserve "Tchórzyno", calcareous fen, leg. H. Więcław 2010 (**). AC27: Żydowo, wet meadow, leg. W. Bacieczko 1989 (SZCZ); Niepołcko, meadow, leg. M. Myśliwy 2001 (SZUB); 1.5 km W of Janowo, dried fish pond, leg. M. Myśliwy 2001 (SZUB). AC35: Mironów, Lake Chłop, meadow, leg. B. Kurnicki 2010 (SZUB). AC45: $\mathrm{N}$ edge of Lake Kozie, periodically waterlogged meadow, leg. M. Wayda 2009 (KRA). AC48: Lipy, planned nature reserve "Lilie Wodne”, peatland, leg. M. Myśliwy 2000 (SZUB). BB07: W of road Polanów-Krąg, calcareous fen in forest, leg. J.

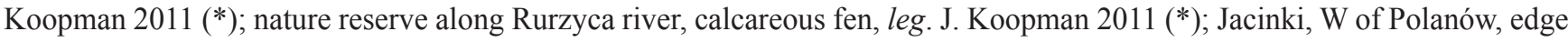
of peatland, leg. J. Koopman 2011 (*). BB61: Zagórzyce, edge of emergent vegetation leg. M. Wilhelm 2011 (SZUB). BB94: Jabłonowo, leg. W.R. 1876 (TRN). BC00: Kiełpino near Choszczno, forest meadow, leg. H. Więcław 2011 (**).

\section{Carex viridula var. viridula}

Dolnośląskie. BE16: Pełczyn, moist meadow, leg. A. Krawiecowa 1964 (WRSL). BE36: Przedmoście Święte, fen, leg. W. Stojanowska 1965 (WRSL). BE64: Olszany near Strzegom, wet meadow, leg. Łukasik 1969 (LOD).

Kujawsko-Pomorskie. CB65: Woziwoda, Lake Sztuczne, leg. M. Boiński \& M. Ceynowa-Giełdoń 1968 (TRN). CB68: N of village Łążek, edge of Lake Piaseczno, leg. H. Więcław 2009 (**). CC15: Szczutki, wet meadow, leg. E. Krasicka-Korczyńska 1992 (BYDG). CC67: Mątwy, forest patch along Noteć river, leg. Ł. Banach 1969 (LOD). CC87: Przyjezierze, forest, leg. W. Gugnacka 1971 (TRN); Przyjezierze, Lake Czyste, leg. W. Gugnacka 1971 (TRN). CC88: Siemionki, Lake Gopło, 
meadow, leg. K. Latowski 1976 (POZ). CD19: Zakrzewek near Sompolsk, leg. K. Drymner 1891 (WA). DC01: Wąbrzeźno, edge of Lake Wieczno, leg. K. Stempska 1961 (POZ); Lake Wieczno, leg. M. Ceynowa-Giełdoń \& E. Werling 1968 (TRN). DC11: Ryńsk, lake edge, leg. M. Ceynowa 1961 (TRN). DC42: Obrowo, depression between dunes, leg. K. Kępczyński 1965 (TRN). DC43: Liciszewy Lipieńskie, edge of Lake Lipień, leg. A. Zalewski 1891 (WA). DC45: Ląkie, leg. K. Kępczyński 1967 (TRN). DC55: Wólka, fen, leg. M. Sokół 1959 (TRN); Wólka Duża, leg. K. Kępczyński 1960 (TRN). DC56: Modrzewie, meadow, leg. K. Kępczyński 1960 (TRN). DC63: Grabiny, leg. K. Kępczyński 1960 (TRN). DC64: Ostrowite near Lipno, meadow, leg. A. Zalewski 1890 (WA).

Lubelskie. FD48: Przychody, poor fen, leg. D. Fijałkowski 1965 (LBLM). FD57: Lipniaki, swamp in forest, leg. D. Fijałkowski 1965 (LBLM). FD64: Huta Dąbrowa near Adamów, fen, leg. D. Fijałkowski 1965 (LBLM). FD74: Zagórze, poor fen, leg. D. Fijałkowski 1965 (LBLM). FD99: Ostrów Lubelski, meadows along Tyśmienica river, leg. D. Fijałkowski 1951, 1964 (LBLM). FE03: Puławy, moist meadow near railway station, leg. B. Polakowski 1953 (OLTC); Gołą, ditch, leg. A. Gelo 1972 (LBLM). FE06: Samoklęski, moist pine forest, leg. J. Motyka 1946 (LBLM). FE84: Zaklików, meadow, leg. H. Koprowska 1925, 1926 (LBLM). FE97: Filisy, edge of poor fen, leg. D. Fijałkowski 1965 (LBLM). FE98: Frampol, edge of reservoir, leg. B. Oleszek 1989 (KRA, LBLM). FF08: Błoto Obary, poor fen, leg. T. Krzaczek 1961 (LBLM). FF18: Żary near Wólka Biska, wet sand near road, leg. A. Nobis 2005 (KRA); Gózd Lipiński, moist meadow, leg. A. Michalewska 2003 (KRA). FF28: Potok Górny, ditch, leg. A. Michalewska 2003 (KRA); Luchów Dolny, meadow, leg. A. Michalewska 2003 (KRA). GD40: Danówka, Bagno Zieleniec (marsh), leg. D. Fijałkowski 1965 (LBLM). GD53: Wiski, swamp in forest, leg. D. Fijałkowski 1962 (LBLM). GE00: Ostrów Lubelski, Lake Uścimowskie, leg. D. Fijałkowski 1956 (LBLM). GE01: poor fen near Lake Łukie, leg. D. Fijałkowski 1951 (LBLM); Wytyczno, nature reserve "Krowie Bagno", mire, leg. D. Fijałkowski 1951 (LBLM), leg. I. Dąbkowska 1938 (KRAM), leg. M. Szewczyk 1978 (KRAM); Wola Wereszczyńska, Lakes Karaśne and Płatyczne, leg. D. Fijałkowski 1956 (LBLM); Wola Wereszczyńska, poor fen near Lake Długie, leg. I. Iwaniak \& T. Kuszpit 1957 (LBLM); Wólka Wytycka, bog, leg. D. Fijałkowski 1957 (LBLM); Wytyczno, peatland near Lake Wytyckie, leg. D. Fijałkowski 1958 (LBLM); edge of Lake Moszne, leg. E. Hoteja 1978 (KRAB). GE02: Wólka Wytycka, peatland near Lake Długie, leg. L. Fagasiewicz 1964 (LOD). GE03: Hańsk, leg. F. Kwieciński 1889 (WA). GE35: Brzeźno, calcareous fen, leg. D. Fijałkowski 1960 (LBLM); Ostrów near Chełm, wet birch forest, leg. I. Dąbkowska 1938 (KRAM). GE84: Miączyn, meadow, leg. D. Fijałkowski 1951 (LBLM). GE90: Hedwiżyn, SE part of peatland Tałandy, leg. H. Piotrowska 1957 (POZ). GF11: Józefów, edge of Lake Tarnowolskie, leg. A. Sokołowski 1965 (BIL).

Lubuskie. AD55: 500 m SW of Stróżki, edge of Lake Jańsko, leg. P. Kobierski 2009 (*). AD64: 2 km W of Osiek, ecological area "Bagna przy Rabym Kamieniu", former sites of peat extraction, leg. P. Kobierski 2009 (*); ecological area "Ruskie Stawy”, abandoned fish ponds, leg. P. Kobierski 2009 (*); S of Kolonia Dlużek, abandoned fish pond, leg. P. Kobierski 2012 (*); 1 km of Chełm Żarski, peatland at edges of fish pond Chełmno, leg. P. Kobierski $2011\left(^{*}\right)$. AD65: E of Janowice, meadow, leg. J. Serwatka 1963 (SZUB); SE of Kolimin, near gravel pit, leg. M. Ciaciura 2006 (SZUB); S of Lubsko, moist places at former extraction sites, leg. M. Ciaciura 2007 (SZUB); Lubsko, clay pits near Artyleryjska Street, leg. P. Kobierski $2009\left(^{*}\right)$; Lubsko, edges of dam reservoir, leg. P. Kobierski $2013(*)$. AD75: ecological area "Bagna Jasieńskie", former sites of peat extraction, leg. P. Kobierski 2008 (*). AD85: W of Żary, silty edges and bottoms of former gravel pits of timber-processing company Kronopol, leg. R. Ryś 2012 (*); 1 km SE of Miłowice, peaty pool, leg. R. Ryś 2013 (*). AD86: 0.5 km W of ŻaryKunice (Glass Works), edges of partly overgrown lignite mining pits, leg. R. Ryś 2013 (*); SE of Żary-Kunice, flooded clay pit Kopara, leg. R. Ryś 2013 (*). AD95: S of Mirostowice Górne and Stawniki, peatlands near A4 highway, leg. R. Ryś 2013 (*). AD96: Jankowa Żagańska, former peat extraction site and edges of peatland under a power line, leg. R. Ryś 2013 (*).

Lódzkie. DD27: Dobrzelin, wetland, leg. B. Wojtczak 1954 (LOD). DD43: Wichrówek, meadow, leg. S. Tomaszewski 1955 (LOD). DD44: Ozorków, most meadow, leg. I. Dziwisz 1955 (LOD). DD68: Kołacinek, wet meadow, leg. Z. Antosiak \& I. Gratel 1967 (LOD). DD74: Bechcice, meadow, leg. M. Grabowska 1949 (LOD); Zofiówka near Lutomiersk, meadow, leg. E. Ubysz 1968 (LOD). DD81: Miłobądź, mire, leg. J. Bogusławski 1949 (LOD). DD95: Pabianice, meadow and ditch, leg. Z. Pawelec 1994 (KRAB). DE01: Sokołów, wet pasture, leg. T. Załuski 1972 (LOD). DE14: Zbyszek, mire, leg. L. Kucharski \& W. Przybylińska 1978 (LOD). DE21: Szynkielów, wet meadow, leg. I. Góźdź 1977 (LOD). DE23: Pawłów, torfowisko przejściowe, leg. M. Mamiński 1982 (LOD). DE24: Chmielowiec near Szczerców, mire, leg. J. Hereźniak 1963 (LOD); Józefino, meadow on Widawka river, leg. J. Hereźniak 1963 (LOD); Lubiec, poor fen, leg. M. Jędrzejczak 1964 (LOD); Zarzecze, peat extraction site, leg. J. Hereźniak 1965 (LOD); Żabczanka, meadow, leg. J. Hereźniak 1966 (LOD); Zarzecze, peatland on Widawka river, leg. J. Hereźniak 1968 (LOD). DE27: Piaski, moist meadow on Widawka river, leg. J. Hereźniak 1964 (LOD); Piaski, forest mire, leg. E. Gabryszewska \& H. Marcinkowska 1978 (LOD). DE31: Strugi near Kochlew, meadow S of Florian's Spring, leg. H. Więcław $2010\left(^{*}\right)$. DE36: Kurzymąka, part of village Seweryn, former site of peat extraction, partly overgrown, leg. M. Mamiński 1983 (LOD). DE41: W of Bobrowniki, edge of Żabi Staw (pond), leg. H. Więcław 2010 (*). DE42: Wydrzynów near Pajęczno, edge of wetland, leg. J. Kaszuba 1965 (LOD). DE44: Dubidze, mire, leg. I. Błaszczyk 1960 (KRA). DE47: Kodrąb, meadow on Widawka river, leg. J. Hereźniak 1965 (LOD). DE49: Bąkowa Góra, fen, leg. P. Witosławski 1981 (LOD). DE50: Kałuże, mire, leg. St. Grzebiela 1975 (LOD). DE59: Przedbórz on Pilica river, leg. J. Lembke 1947 (LOD); Wojciechów, mire, leg. H. Leder 1970 (LOD). DE66: Jasień, forest reserve with yew, thicket, leg. E. Cajgler 1958 (LOD). ED41: Bolimów Forest, drained peatland, leg. W. Pisarek 1983 (LOD). ED62: Fryszerka-Henryków, poor fen, leg. H. Urbanek 1967 (LOD). EE00: Tomaszów Mazowiecki, nature reserve "Niebieskie Źródła", meadow, leg. 
J. Mowszowicz \& R. Olaczek 1960 (LOD). EE11: Sobawiny, moist forest path, leg. A. Trojecka-Brzezińska 2010 (KRA). EE12: Bielowice, moist meadow, leg. A. Trojecka-Brzezińska 2010 (KRA); Wola Załężna, moist sports field, leg. A. Trojecka-Brzezińska 2010 (KRA). EE31: Bedlno, wet meadow, leg. J. Walas 1929 (KRA). EE50: Borowa, nature reserve "Piskorzeniec", mire, leg. A. Ucińska 1968 (LOD).

Małopolskie. DF37: Zarzecze, fen near railway station, leg. B. Babczyńska-Sendek 1980 (*); Cieślin-Pustki, poor fen on Sączenica river, leg. B. Babczyńska-Sendek 1981 (*); Golczowice-Borek, at border with Cieślin and Pustki, poor fen, leg. B. Babczyńska-Sendek 1981 (*). DF38: W of Wolbrom, meadow, leg. B. Babczyńska-Sendek 1981 (*). DF46: Bukowno, meadow, leg. K. Jędrzejko \& J. Żarnowiec 1980 (KRA); Chełmek, oxbow lake, leg. J. Zalewska 1996 (KRA). DF55: Chrzanów, wet meadow, leg. L. Olesiński 1972 (KRAM). DF59: Prądnik Korzkiewski, wet meadow, leg. unknown 1964 (KRAM). DF69: Wieliczka, moist meadow, leg. J. Żelazny 2005 (KRA); Kraków-Kostrze, leg. P. Kalinowski 2007 (*). DF79: Podgórki near Tyniec (Kraków), peaty meadows at bases of limestone hills, leg. A. Jasiewicz 1966 (KRAM). DG16: Babia Góra leg. H. Zapałowicz 1879 (KRAM). DG18: N of Sidziny, fen in groundwater seepage area at spruce forest edge, $630 \mathrm{~m}$ a.s.1., leg. M. \& J. Guzikowie 1964 (KRAM); Sidzina, wet meadow, leg. B. \& L. Stuchlikowie 1950 (KRAM). DG27: Zubrzyca Dolna, between Durczakowa II and Groń, wet meadow, 690 m a.s.l., leg. M. Guzikowa 1959 (KRA); Lipnica Mała, N part of village, peatland, 755 m a.s.1., leg. M. \& J. Guzikowie 1966 (KRAM); Lipnica Wielka, moist depression on fluvial terrace of Lipnica stream, 610 m a.s.l., leg. M. \& J. Guzikowie 1966 (KRAM); Jabłonka, moist pasture near Zubrzyca stream, 625 m a.s.1., leg. M. \& J. Guzikowie 1966 (KRAM). DG28: S of Podwilk, Kuligowa Góra (Kuligówka), waterlogged, clayey depression near field road, 750 m a.s.l., leg. M. \& J. Guzikowie 1966 (KRAM). DG59: Łysanki, leg. I. Król 1913 (KRA); above mountain pass Przełęcz na Patykach (now Przełęcz Białego) leg. I. Król 1914 (KRA); Molkówka (montane glade), Podtatrze, leg. I. Król 1914 (KRA); Dolina Strążyska (valley), leg. I. Król 1914 (KRA); Polana Biały Potok (glade), fen in groundwater seepage area, leg. A. Jasiewicz 1953 (KRAM); eastern peak of Łysanki, 1187 m a.s.1., leg. unknown 1881 (KRAM). EF32: Słupów, moist meadows in Nidzica river valley, leg. W Bartoszek 2005 (KRA). EF70: Bogucice, pond edge, leg. R. Grusz 1983 (KRA). EF71: Podłęże near Niepołomice, wet meadow, leg. A. Jasiewicz 1951 (KRAM). EG01: Góra Ćwilin (mount) near Jurków, moist places near road, 620 m a.s.l., leg. K. Towpasz 1968 (KRA). EG10: N slope of Luboń Wielki, wet meadow near forest, 670 m a.s.1., leg. unknown 1967 (KRA). EG12: Okrąg (mount), moist places near road, 490 m a.s.1., leg. K. Towpasz 1970 (KRA). EG20: Dolina Mały Kowaniec (valley) under Turbacz (mount), meadow along stream, leg. A. \& J. Kornasiowie 1959 (KRAM). EG23: Kamieńczyk, moist meadow and roadside ditch, 420 m a.s.1., leg. K. Stawowczyk 2006 (KRA). EG31: lower terrace of Dunajec river near Knurów, on silt in a moist depression, 540 m a.s.l., leg. J. Kornaś 1974 (KRA); Pieniny Rock Belt, under Kramnica, fen in groundwater seepage area, leg. K. Grodzińska 1961 (KRAM); Skalice Spiskie near Frydniański stream, fen in groundwater seepage area, leg. K. Grodzińska 1964 (KRAM). EG32: Maniowy, forest fen in groundwater seepage area and wetland near old Dunajec river bed, 520 m a.s.1., leg. A. \& J. Kornaś 1962 (KRA). EG33: Krościenko, wet meadow near stream, leg. L. Fagasiewicz 1950 (LOD); valley of Skotnicki Potok (stream), 510 m a.s.l., leg. K. Stawowczyk 2008 (KRA). EG41: Czarna Góra near Białka Tatrzańska, leg. I. Król 1905 (KRA). EG47: Wojkowa, wet meadow, leg. M. Wójcik 1996 (KRAB). EG50: Kalatówki above Kuźnice, in dwarf mountain pine zone, leg. M. Raciborski 1911 (KRA); Kalatówki, under mountain pass Przełęcz na Patykach (now Przełęcz Białego), leg. I. Król 1914 (KRA).

Mazowieckie. DC46: Blinno, leg. K. Kępczyński 1961 (TRN); Szczutowo, lake edge, leg. K. Kępczyński 1967 (TRN). DC59: Koziebrody, moist meadow, leg. A. Ejsmond 1886 (WA). ED05: Chotomów, fen, leg. B. Tymak 1937 (WA). ED09: Klembowo, nature reserve "Dębina”, ditch, leg. B. Chruszczewska1962 (LOD). ED15: Laski, meadow, leg. K. Nowak 1966 (WA). ED17: Rembertów, moor, leg. W. Żukowski 1963 (POZ). ED25: Ożarów Mazowiecki, grassland on clay pits, leg. K. Nowak 1966 (WA). ED28: Stara Miłosna, meadow, leg. St. Małysz 1979 (WA). ED34: Podkowa Leśna, meadow, leg. K. Nowak 1971 (WA). ED37: Natolin, meadow, leg. unknown 1869 (WA), Natolin, leg. unknown 1969 (POZ). ED48: Całowanie, mire, leg. J. Zielińska 1951 (WA). ED49: Całowanie, alder thicket, leg. K. Nowak 1953 (WA). ED93: Różanna, riverine meadow, leg. A. Stańczak-Jażdżuk 1997 (LOD). EE27: Dąbrówka Warszawska near Wierzbica, pond edge, leg. M. Nobis 2003 (KRA). EE35: 1 km SW of Koszarów, roadside, leg. M. Nobis 2003 (KRA); 1 km W of Szydłowiec, fen, leg. M. Nobis 2003 (KRA); Budki I near Szydłowiec, moist meadow, leg. M. Nobis 2003 (KRA); 1 km NNW of Książek, forest meadow, leg. M. Nobis \& A. Michalewska 2003 (KRA); Budki III near Szydłowiec, roadside, leg. M. Nobis 2003 (KRA); 3 km SW of Szydłowiec, moist road, leg. M. Nobis 2003 (KRA). EE36: Józefów, N of Szydłowiec, ditch, leg. M. Nobis 2003 (KRA). EE37: $2 \mathrm{~km} \mathrm{~W}$ of Pakosław near Iłża, wet meadow, at fen edges, leg. M. Nobis 2003 (KRA); Wierzbica, N part of quarry, near pond, leg. M. Nobis 2003 (KRA); NW of Wierzbica near Radom, drying waterhole in quarry, leg. M. Nobis 2004 (KRA). EE38: Pakosław, mire, leg. G. Sosnowska 1954 (KRA); Płudnica near Wierzbica, fish pond, leg. M. Nobis 2003 (KRA). FC74: Nowa Wieś, moist field, leg. Z. Głowacki 1989 (WSRP). FC75: Chądzyń, leg. P. Kalinowski 2010 (*). FC86: Kolonia Wieska, leg. P. Kalinowski 2010 (*). FC91: Myszadła, peat dyke, leg. unknown 1961 (LOD). FD07: Korczew NE, leg. P. Kalinowski 2005 (*); Laskowice, leg. P. Kalinowski 2013 (*). FD08: Ruska Strona, leg. P. Kalinowski 2004 (*). FD18: Kolonia Niemojki, leg. P. Kalinowski 2013 (*). FD25: Golice, ditch, leg. D. Jówko 1973 (LBLM). FD35: Wólka Wiśniewska, meadow, leg. D. Jówko 1973 (LBLM).

Opolskie. CE67: Czaple Wolne, wet meadow, leg. A. Sendek 1968 (OPOL). CE95: Gosławice, leg. K. Bialucha 1937 (OPOL). CF07: N of Izbicko, pond, leg. M. Ciaciura 1964 (SZUB). CF13: NE of Dobrzyków, meadow, leg. E. Kuźniewski 1967 (SZUB). 
Podkarpackie. FF16: 750 m S of W end of Ryczka, sandy and moist field roadside, leg. A. Michalewska 2004 (KRA); near N end of village Bieliny, moist sandy roadside, leg. A. Michalewska 2004 (KRA); 500 m SSE of village Glinianka, pond, leg. A. Michalewska 2004 (KRA). FF17: Sieraków, about $750 \mathrm{~m} \mathrm{~W}$ of end of village, partly overgrown oxbow lake of Tanwia river, leg. A. Michalewska 2004 (KRA); 1 km N of E end of Nowa Wieś near Krzeszów Górny, moist meadow, leg. A. Michalewska 2004 (KRA). FF23: Spie near Wilcza Wola, forest edge near moist meadow, leg. A. Wołowicz 1990 (KRAM). FF26: E of Krzeszów, moist roadside ditch, leg. A. Michalewska \& M. Nobis 2003 (KRA). FF27: Sigiełki, near a sand pit, leg. A. Michalewska 2003 (KRA); Sigiełki, moist meadow, leg. A. Michalewska 2003 (KRA); Krzeszów Górny, moist pit, leg. A. Michalewska 2003 (KRA); NE of S end of village Łazów, meadow edge, near a ditch, leg. A. Michalewska 2004 (KRA). FF28: Dąbrówka, moist meadow near alder wood, leg. A. Michalewska 2003 (KRA). FF38: N end of village Kolonia Polska, moist meadow, leg. A. Michalewska 2004 (KRA); 1.5 km N of village Cieplice Górne, alder forest, leg. A. Michalewska 2004 (KRA). FF48: Pigany, moist sand pit, leg. A. Michalewska 2003 (KRA); Pigany, near oxbow lake of San river, sand pit near settlement Kępa, leg. A. Michalewska \& M. Nobis 2004 (KRA). FF50: Nagoszyn, ditch edge in farmland, leg. M. Wayda 1990 (KRA). FF58: Jagiełła near Przeworsk, waterlogged pasture near forest, leg. Ralyn 1971 (KRA). FF60: Nagawczyna, emergent vegetation, leg. K. Towpasz 1978 (KRA). FG01: Łężyny, wet meadow, leg. K. Oklejewicz 1989 (KRA). FG14: Besko, pasture on gravelly edge of Wisłok river, leg. K. Oklejewicz 1989 (KRA). FG16: Sanok near a cemetery, meadow, leg. unknown 1922 (KRAM). FG35: N of Komańcza, wet place in quarry, Jawornik, leg. A. Nobis \& M. Nobis 2006 (KRA). FG47: Tyskowa near Łopienka, fen in groundwater seepage area, leg. A. Jasiewicz 1962 (KRAM). FG59: Stuposiany, fen in groundwater seepage area, leg. A. Jasiewicz 1960 (KRAM). FG79: Wołosate, fen in groundwater seepage area above Wołosatka river valley, leg. B. Zemanek 1993 (KRA). GG70: Wołosate, Przełęcz Beskid (mountain pass), roadside ditch, leg. H. Więcław 2010 (**).

Podlaskie. FA78: Wiżajny, mire, leg. A. Kawecka 1969 (BIL). FA88: Jałowo, meadow, leg. A. Sokołowski 1969 (BIL). FA95: Czarne, edge of Lake Czarne, leg. N. Romańczuk 1971 (BIL). FA96: Krzywólka, edge of Lake Krzywe, leg. A. Pliszko 2008 (KRA); Białe Jeziorki, wet coniferous forest, leg. A. Pliszko 2010 (KRA). FA97: Morgi, springhead, leg. A. Pliszko 2010 (KRA); Bachanowo near Hańcza, springhead near Czarna Hańcza river, leg. A. Sokołowski 1975 (BIL); Błaskowizna, peaty meadow, leg. A. Sokołowski 1975 (BIL); Błaskowizna, edge of Lake Boczniel, leg. A. Kawecka 1969 (BIL). FA99: Przejma Wielka near Suwałki, meadow, leg. A. Kawecka 1986 (BIL). FB06: Szafranki, most meadow, leg. A. Pliszko 2011 (*). FB07: Piecki, edge of Lake Okminek, leg. A. Pliszko 2009 (KRA), Zusno, former peat extraction site in wet coniferous forest, leg. A. Pliszko 2008 (KRA), Sokołowo, abandoned, waterlogged gravel pit, leg. A. Pliszko 2010 (KRA). FB08: Prudziszki, mire, leg. A. Kawecka 1969 (BIL). FB09: Krzywe near Suwałki, mire, leg. A. Sokołowski 1976 (BIL); Ryżówka, mire, leg. A. Sokołowski 1981 (BIL); Ryżówka, mire near Lake Dowcień, leg. A. Sokołowski 1976 (BIL). FB17: Kamionka Stara, edge of Lake Okragłe in Rospuda river valley, leg. A. Pliszko 2008 (KRA). FB27: Lipówka, pasture near a waterhole, leg. A. Pliszko 2011 (KRA). FB39: peatland on Rospuda river, Szczebra Forest District, forest section 130, leg. A. Sokołowski 1987 (BIL). FB47: Rajgród, poor fen near Lake Rajgrodzkie, leg. L. Fagasiewicz 1953 (LOD); Rajgród, leg. L. Olesiński 1957 (OLTC). FB49: Kolnica, meadow, leg. J. Wołkowycki 1971 (BIL); Polki, moist meadow near Lake Kolno, leg. S. Fieńko 1971 (BIL); Gliniski, peaty meadow, leg. J. Żurawski 1971 (BIL). FB58: Orzechówka, mire, leg. A. Sokołowski 1971 (BIL). FB65: Dybła, meadow, leg. A. Kawecka 1970 (BIL). FB66: Szymany, Bagno Kurasy, leg. W. Matuszkiewicz 1953 (WA). FC02: Nowogród, meadow, leg. H. Sielska 1935 (WA). FB69: Polkowo, moist depression, leg. J. Żurawski 1971 (BIL). FB95: Karwowo, peaty meadow, leg. A. Sokołowski 1968 (BIL). FC04: Bronaki-Olki, SW of Jedwabne, poor fen, leg. A. Sokołowski 1992 (BIL). FC05: Jedwabne, wet meadow, leg. A. Sokołowski 1968 (BIL); Górki, forest meadow, leg. A. Sokołowski 1969 (BIL). FC25: Kossaki, mire, leg. J. Żurawski 1970 (BIL). FC36: Puszcza Gołaszcze, moist meadow, leg. A. Sokołowski 1970 (BIL). FC98: Zajęczniki E, leg. P. Kalinowski 2013 (*). GB00: Krasnopol, moist meadow, leg. A. Sokołowski 1969 (BIL); Michnowce, pit (extraction site) on Czarna river, leg. S. Fieńko 1969 (BIL); Stabieńszczyzna, meadow, leg. A. Rusińska 1969 (BIL). GB01: Sejny, mire, leg. E. Puławska 1978 (WA); Berżniki, Lake Gajlik, meadow on W edge of lake, leg. E. Jabłońska 2002 (WA); Dworczyska, mire, leg. J. Żurawski 1971 (BIL). GB10: Zakąty, lake edge, leg. A. Sokołowski 1971 (BIL). GB11: Giby, moist meadow near Lake Dowcień, leg. B. Sudnik 1975 (WA); Giby, Lake Dowcień, leg. E. Mazek 1975 (WA); Giby, damp depression among meadows, leg. W. Mazur 1976 (WA); Białogóry, edge of Bobrowe Bagno (bog), leg. T. Szarejko 1984 (OLTC); Giby, peatland surrounded by fields near road Giby-Sejny, leg. T. Szrejko 1987 (LBLM). GB22: near Augustów, forest edge close to Lake Brożane, leg. M. Sychowa 1963 (KRAM). GB30: Przewięź, mire, leg. A. Sokołowski 1971 (BIL). GB31: Jazy near Mikaszówki, mire, leg. A. Sokołowski 1971 (BIL); nature reserve "Perkuć", leg. A. Sokołowski 1972 (BIL). GB83: Szyszki, peaty meadow, leg. N. Romańczuk 1969 (BIL). GC24: Gorbacze, peaty meadow, leg. A. Sokołowski 1973 (BIL). GC30: Dołki, leg. J. Żurawski 1970 (BIL). GC31: Laszki, moist meadow, leg. A. Sokołowski 1967 (BIL). GC32: Żywkowo, moist meadow, leg. A. Sokołowski 1988 (BIL). GC33: Hajdukowszczyzna, fen in groundwater seepage area, leg. A. Sokołowski 1978 (BIL); Rohozy, meadow, leg. A. Sokołowski 1968 (BIL). GC34: Eliaszuki, meadow, leg. J. Żurawski 1971 (BIL). GC35: Narew, near railway station Siemianówka, leg. W. Mieńko \& G. Magiera 1981 (BSG). GC41: Chraboły, moist depression, leg. A. Kawecka 1969 (BIL). GC42: Ciełuszki, most meadow, leg. A. Sokołowski 1967 (BIL). GC44: Ladzka Puszcza (forest), forest section 58C, mire, leg. A. Sokołowski 1977 (BIL). GC45: Janowo, peaty meadow, leg. A. Sokołowski 1973 (BIL); Ladzka Puszcza (forest) near Gnilca, forest section 55B, leg. A. Sokołowski 1977 (BIL). GC63: Mochnate, moist depression, leg. A. Sokołowski 1968 (BIL). GC70: Chranibory, thicket on wet meadow, leg. A. Sokołowski 1968 (BIL). GC72: Kleszczele, thicket, leg. I. Dąbkowska 1938 (KRAM). GC73: Nikiforowszczyzna, forest 
section 694D, leg. A. Sokołowski 1999 (BIL). GC82: Czeremcha, leg. A. Sokołowski 1985 (BIL). GC91: Wilanowo, moist meadow, leg. A. Sokołowski 1967 (BIL).

Pomorskie. BB28: Bobęcin, sandy edge of Lake Bobęcińskie Wielkie, leg. I. Dąmbska 1972 (POZ). CA36: Białogóra, forest road, leg. K. Latowski 1975 (POZ); Białogóra, moist depression between dunes among moors, leg. H. Piotrkowska 1968 (UGDA); Piaśnica, sandy edge of a canal, leg. H. Piotrkowska 1968 (UGDA); Białogóra, depression between dunes, leg. M. Herbichowa 1969 (UGDA); leg. H. Więcław 2009 (**). CA38: nature reserve "Bielawskie Błoto", in NE part of peatland near road Mieroszyno-Ostrowo, leg. J. Mondelska 1949 (POZ); Bielawskie Błoto near Czarny Młyn, moist sand, leg. M. Herbichowa 1971 (UGDA); 2 km NE of Sławoszyno, near nature reserve "Bielawskie Błoto", leg. M. Ciaciura 2004 (SZUB); E of Sławoszynko, forest near nature reserve "Bielawa", leg. H. Więcław 2010 (**). CA41: Smołdziński Las, meadow and edge of Lake Dołgie Wielkie, leg. unknown 1992 (SPNH). CA42: Łeba, Łeba Spit (Mierzeja Łebska), depression between dunes, leg. H. Więcław 2011 (**). CA49: Wielka Wieś, moist pasture near Bay of Puck leg. H. \& T. Tacik 1962 (KRAM). CA58: Reda, leg. von Klinggraeff no date (TRN). CA59: Osłonino, nature reserve "Beka”, low-sedge fen S of village, leg. H. Więcław 2009 (**). CA96: Łączyno, mire, leg. M. Jakkalska 1982 (UGDA). CA98: sandy edge of Lake Karlikowskie, leg. S. Lisowski 1960 (POZ); Borowo, between Kartuzy and Żukowo, SE edge of Lake Klebarskie, leg. A. Jutrzenka-Trzebiatowski 1969 (OLTC); Borowa, edge of Lake Karlikowo, leg. Krzywański 1970 (LOD); Lake Karlikowo, leg. E. Koteja 1969 (KRAM). CB05: Ostrowite, meadow, leg. A. Zalewski 1890 (WA). CB13: Czarna Dąbrowa, sandy edge of Lake Czarne Dąbrówno, leg. I. Dąbska 1955 (POZ). CB22: Lipnica, edge of Lake Kiedrowickie, leg. S. Lisowski 1965 (POZ); Lipnica, leg. M. Rejewski 1965 (TRN); edge of Lake Czarne near forester's lodge Kiedrowice, leg. S. Lisowski 1965 (KRA); Zapceń, small waterlogged site near Bagno Zapceń in direction of Kiedrowice, leg. S. Lisowski 1965 (POZ). CB26: Wdzydze Tucholskie, at N edge of Lake Czyste, leg. F. Szafrański \& K. Tobolski 1966 (POZ); Wdzydze Tucholskie, at S edge of a lake located E of Lake Krzywe, leg. F. Szafrański \& K. Tobolski 1966 (POZ); Wdzydze Tucholskie, E edge of Lake Krzywe, leg. F. Szafrański \& K. Tobolski 1966 (KRA); Wdzydze Tucholskie, E edge of Lake Grzybno, leg. F. Szafrański \& K. Tobolski 1966 (POZ); $\mathrm{S}$ of road Wdzydze Kiszewskie-Gołuń, ecological area "Kiszewskie Bagno" near N edge of Lake Gołuń, poor fen, leg. H. Więcław 2008 (**). CB30: Koczała, Lake Rosko Wyschłe, leg. B. Dobrańska 1978 (TRN). CB31: Upiłka, Lake Babie, leg. M. Rejewski 1965 (TRN); Nowa Brda, Lake Krucze, leg. M. Dembińska 1978 (TRN). CB33: edge of Lake Zmarłe, leg. A. Sokołowski 1961 (BIL); Laski, alluvia along Zbrzyca river, near Lake Czarne, leg. F. Szafrański \& K. Tobolski 1966 (POZ); Lake Stawek near Asmus, leg. M. Ceynowa-Giełdoń \& A. Szczygłowska 1967 (TRN); Swornegacie, edge of Lake Nawionek, leg. M. Ceynowa-Giełdoń 1967 (TRN), leg. M. Wayda 2006 (KRA); Swornegacie, edge of Lake Gardliczno Duże, leg. K. Ciura 2008 (KRA); Laski, Lake Żabionek, wet meadow, leg. L. Świerczyńska \& M. Korczyński 1994 (BYDG). CB34: Lake Warszyn, leg. M. Boiński 1966, 1967 (TRN), leg. K. Kozłowska, leg. M. Wayda 2006 (KRA), leg. Sz. Drobniak 2008 (KRA). CB35: Lake Zmarłe, N of Lubnia, leg. M. Rejewski 1966 (TRN); Lake Kły, N of Lubnia, leg. M. Rejewski 1966 (TRN). CB42: Funka, edge of Lake Głuche Małe, leg. M. Korczyński 1993 (BYDG). CB43: Drzewicz, Lake Wielkie Krzywce, leg. M. Rejewski 1964 (TRN); Męcikał, E end of Lake Jeleń, on wet sand, leg. S. Lisowski 1966 (POZ); W of Męcikał, edge of Lake Zielone, leg. M. Rejewski 1966 (TRN); Drzewicz, edge of Lake Krzywice Małe, leg. M. Korczyński 1993 (BYDG); Lake Jeleń, sandy lake edge, leg. E. Krasicka-Korczyńska 1993 (BYDG). CB44: Giełdoń, edge of Lake Okręglik, leg. S. Lisowski \& F. Szafrański 1965 (POZ); lake near Szotowa Góra (hill), leg. M. Boiński 1966 (TRN); Szczawiny, leg. M. Ceynowa-Giełdoń \& G. Szudelska 1967 (TRN). CB55: Lukowo, wetland, leg. Z. Chirek 1960 (LOD). CB56: Czersk, W edge of Lake Ostrowite, leg. F. Szafrański, K. Tobolski 1966 (POZ); Czersk, S edge of Ostrowite, leg. R. Borys 1969 (POZ).

Śląskie. DE82: Blachownia, sandy depression among meadows, leg. J. Hereźniak 1972 (LOD); Blachownia, emergent vegetation, leg. J. Hereźniak 1972 (LOD); Aleksandria I, fen in groundwater seepage area in river valley near bridge, leg. J. Hereźniak 1975 (LOD); Blachownia, meadow, leg. Botanical Section (Sekcja Botaniczna), L. Samosiej 1988 (LOD); Blachownia, moist meadow near a pond, leg. J. Hereźniak 1988 (LOD). DE83: Łojki, meadow, leg. B. Gajos-Kędzierska 1957 (KRA). DE84: Częstochowa Dąbie, edge of waterhole, leg. I. Kamińska 1997 (LOD). DE94: Korwinów, wet meadow, leg. I. Błaszczyk 1948 (KRA). DE96: Bystrzanowice, mire, leg. D. Kowalska 1987 (LOD). DF01: Piłka, Jeleniak-Mikuliny, wet meadow, leg. W. Bromer 1970 (LOD). DF05: Myszków-Nowa Wieś, mire, leg. E. Uchnast 1997 (KTU). DF13: Pyrzowice, peatland within airport, leg. T. Nowak 1993 (KTU). DF14: Piwoń near Siewierza, fen, leg. A. Sendek 1972 (KTU). DF19: Żarnowiec near Pilica, mire, leg. B. Chwastowski 1978 (KRAM). DF24: Kuźnica Warężyńska, anthropogenic fen in groundwater seepage area in old sandpit, leg. A. Błońska 2011 (*). DF25: Poręba near Zawiercie, poor fen, leg. A. Błońska 2009 (*). DF33: Sosnowiec, mire, leg. A. Sendek 1977 (KRA). DF34: Sosnowiec, leg. A. Sendek 1974 (KRA); Dąbrowa Górnicza Pogoria I, fen in groundwater seepage area, leg. A. Czylok 1995 (KTU); Dąbrowa Górnicza Pogoria I, anthropogenic fen in groundwater seepage area on sand, leg. A. Błońska 2011 (*). DF35: S of Niegowonice, meadow, leg. B. Babczyńska-Sendek 1988 (*). DF36: S of Dąbrowa Górnicza Błędów, in direction of Laski, poor fen, leg. A. Błońska 2011 (*). DF40: Gliwice, leg. D. Wąsowicz 1875 (KRAM). DF44: Jęzor, between Mysłowice and Jaworzno, at deciduous forest edge, leg. A. Korczyk 1959 (OLTC); Sosnowiec Bory, anthropogenic wetlands in old sandpit, leg. A. Błońska 2011 (*); Jaworzno, sand pit near Jęzor, leg. T. Tacik 1956 (KRAM). DF61: Orzesze-Waszczyce, edge of ditch on meadow near Żorska Street, leg. J. Oleś 1997 (KTU). DG04: Oczków, peaty patch, leg. B. Kotońska 1986 (KRA). DG13: Barania Góra (mount), E slopes, leg. A. Żmuda 1917 (KRA).

Świętokrzyskie. DE69: Kluczewsko, peaty meadow, leg. I. Błaszczyk 1949 (KRA); Żabieniec near Stanowiska, moist depression close to emergent vegetation, leg. H. Więcław 2010 (*). DE78: Silpia Duża, peaty site at pine forest edge, leg. M. 
Bielecki 2009 (KRA). DE79: Niecka Włoszczowska, peatland Wielga, leg. M. Bielecki 2007 (KRA). ); Osicyna near Brzeście, moist meadow, leg. M. Bielecki 2010 (KRA). DE88: Podgóry, moist road in forest, leg. M. Bielecki 2007 (KRA). DF09: Sprowa, peaty meadow, leg. I. Błaszczyk 1949 (KRA). EE32: Bedlenko, fish pond, leg. A. Trojecka-Brzezińska 2010 (KRA). EE36: Szydłówek, wet edge of waterhole, leg. M. Nobis 2003 (KRA). EE37: Wierzbica, limestone quarry, along a ditch, leg. M. Nobis 2002 (KRA). EE45: 2 km NE of Majdów, fen edges, leg. M. Nobis 2003 (KRA); 2 km NE of Majdów, stream edge, leg. M. Nobis 2003 (KRA); Bliżyn near Kielce, moist meadow, leg. H. Piękoś 1966 (KRAM). EE46: Młyn Piaska near Skarżysko Kamienna, wet sand near a pit (extraction site), leg. M. Nobis 2003 (KRA). EE52: SW Smyków, moist thicket, leg. W. Paul 1990 (KRAM). EE55: Suchedniów near Kielce, wet meadow, leg. A. Jasiewicz 1964 (KRAM). EE56: Mostki, drained reservoir, leg. M. Nobis, 2002 (KRA). EE57: forest section 151, Klarnetowo Forest Range, moist sand in a ditch at mixed coniferous forest edge, leg. M. Nobis 2003 (KRA); Starachowice, moist sand near Piachy Reservoir, leg. M. Nobis 2003 (KRA); Starachowice Bugaj, wet clayey slope of spoil tip in forest, leg. M. Nobis 2003 (KRA). EE64: Wzgórza Kostomłockie (hills), N of Górki Szczukowskie, peatland, leg. E. Bróż \& J. Borowiecka 1982 (KTC); Kielce Kostomłoty, fen, leg. I. Kulej \& B. Maciejczak 1986 (KTC); Miedziana Góra, thicket, leg. unknown 1986 (KTC); Zagnańsk near Kielce, waterlogged site in abandoned quarry, leg. M. Nobis 2004 (KRA); NE of Kielce, Zachełmie near Zagnańsk, dried waterhole at bottom of abandoned quarry, leg. M. Nobis 2004 (KRA). EE69: Piaski Kunowskie, fen, leg. R. Piwowarczyk 2003 (KRA). EE70: Belina, fen, leg. W. Pisarek 1989 (LOD); Włoszczowa-Podzamcze, waterlogged peaty glade in forest, leg. M. Bielecki 2009 (KRA). EE71: Wrzosówka, NW of railway station Małogoszcz, poor fen, leg. T. Tacik 1955 (KRA and KRAM). EE75: Grupa Otroczna, moist forest glade, leg. E. Bróż \& M. Chrzanowska 1987 (KTC). EE82: S of SE end of village Mosty near Chęciny, edge of peaty pool (former site of peat extraction), leg. G. Łazarski 2013 (KRA). EE83: Bugaj, meadow and peatland in Bobrza river valley, leg. R. Grumsz 1983 (KRA). EE84: E of Bieleckie Młyny near Morawica, wet sand, leg. G. Łazarski 2013 (KRA). EE91: Cierno near Cacowo (Dziadówki), moist meadow, leg. B. Piwowarski 2008 (KRA); Nagłowice, ponds E of village, moist meadow, leg. B. Piwowarski 2008 (KRA). EE92: Gozna, poor fen, leg. B. Piwowarski 2009 (KRA). EE93: Lasków, moist meadow, leg. B. Piwowarski 2008 (KRA). EF08: Golejów near Staszów, fen in groundwater seepage area near stream, leg. unknown 1973 (KRA). EF14: S of Włochy, anthropogenic depression among meadows, on sandy substrate, leg. H. Więcław 2010 (**); S of Włochy, depression with standing water on meadow, leg. H. Więcław 2010 (**). EF34: Gorysławice, meadows near Nida river, leg. J. Szwagrzyk 1986 (KRAB). EF36: Ostrowce near Nowy Korczyn, moist meadow, leg. B. Chwastowski 1978 (KRAM).

Warmińsko-mazurskie. DA96: Kadyny, mire, leg. J. Szulc 1965 (LOD). DB15: Elblag, Mechnica, leg. E. Straube 1882 (TRN). DB59: Warlity Wielkie, drained bottom of former fish pond, leg. M. Środa 1997 (OLTC). DB76: nature reserve "Karaś", peatland in E part, Lake Karaś, leg. H. Wojciechowska \& B. Polakowski 1954 (OLTC). EB35: Biesówko, pasture and meadow, leg. L. Olesiński 1967, 1968 (OLTC); Zarębiec, wet pasture, leg. L. Olesiński 1967 (OLTC). EB41: Jonkowo, poor fen, leg. L. Olesiński 1956 (OLTC). EB45: Pudlag, leg. U. Boińska 1970 (TRN). EB46: Dymer, birch thicket on former peat extraction sites, leg. L. Olesiński 1971 (OLTC). EB56: Popowa Wola, moist meadow on drained peatland, leg. L. Olesiński 1967 (OLTC). EB65: Linowo, periodically flooded depression on peaty pasture, leg. L. Olesiński 1967 (OLTC); Pasym, Leleszki complex of fish ponds, leg. M. Środa 2001 (OLTC), leg. B. Zawadzka 2001 (OLTC). EB74: Narty, sandy edge of Lake Świętajno, leg. M. Środa 1986 (OLTC). FA84: Gołdap, leg. R. Schultz 1893 (TRN). FB03: Lipowo, meadow, leg. B. Polakowski 1956 (OLTC). FB34: Królowa Wola, meadow, leg. M. Strugała 1971 (BIL). FB52: Lisuny (Łysonie), meadow, leg. L. Olesiński 1958 (OLTC).

Wielkopolskie. BC29: Wielowieś, S edge of Lake Proboszczowskie, leg. J. Dziedzic 1963 (POZ). BC37: Jabłonowo Wlkopolskie, moist meadow, leg. M. Anczyńska 1980 (POZ). BC52: Drawsko, fen at edge of Lake Moczydło, leg. K. Latowski 1975 (POZ). BC54: Miały, meadow near Lake Święte, leg. K. Latowski 1974 (POZ). BC84: Lake Luboszki, mire, leg. S. Lisowski \& F. Szafrański 1966 (POZ). BC94: Zgierzynka, calcareous meadows, leg. W. Żukowski 1975 (POZ); Zgierzynka, leg. K. Latowski \& W. Żukowski 1977 (POZ). BC97: Kiekrz, meadow near small Lake Kiekrzskie, leg. A. Danowska 1932 (POZ); Lake Lusowskie, salt-marsh and ditch edge, leg. K. Latowski \& W. Żukowski 1975 (POZ). BD18: Czapury, leg. M. Miller 1893 (POZ). BD45: Mochy, meadow, leg. J. Paczowski 1932 (POZ). BD49: Dolsk, meadow near N edge of Lake Dolskie Wielkie, leg. R. Kaczmarek 1959 (POZ). CC31: Folusz, meadows near mill, leg. M. Rejewski 1964 (TRN). CC91: damp gravel pit near Lake Jerzyńskie, leg. H. Wojterska 1966 (PUMA, POZ). CD06: Salmonowo, leg. W. Żukowski 1974 (POZ); between Powidz and Anastazewo, edge of Lake Powidzkie, leg. K. Latowski 1978 (POZ). CD28: Wilków, wet meadow, leg. H. Adamiak 1958 (LOD). CD37: Główiew, fen in groundwater seepage area, SE of village, leg. W. Żukowski 1977 (POZ). CD45: Białobłoty, moist, sandy roadside depression, leg. K. Latowski \& W. Żukowski 1975 (POZ). CD58: Orla Góra, forest glade with rock outcrops, leg. M. Raniś 1995 (LOD).

Zachodniopomorskie. AB14: Łowno near Międzywodzie, salt-marshes, leg. B. Bosiacka 2009 (SZUB). AB17: Kusin, between Trzebiatów and Skrobotowo, fen in groundwater seepage area, leg. B. Bosiacka 2012 (SZUB). AB18: Włodawka near Trzebiatów, salt-marsh, leg. H. Więcław 2012 (**). AB22: Wolin, Drożkowe Łąki, leg. H. Piotrowska 1959 (UGDA). AB23: Międzyzdroje, leg. Aschersson 1856 (KRAM); Wolin, SE edge of Lake Wisełka, leg. H. Piotrowska 1962 (UGDA); Ładzin, waterlogged meadow at edges of peaty pools, leg. H. Piotrowska 1962 (UGDA); Wolin, NW edge of Lake Wisełka, leg. H. Piotrowska 1962 (UGDA); Wisełka, E edge of Lake Wisełka, leg. H. Piotrowska 1963 (UGDA); Międzyzdroje, peaty meadow, leg. E. Kiecor 1966 (LOD); W edge of Lake Wisełka, leg. H. Piotrkowska \& R. Markowski 1966 (UGDA). AB24: Kołczewo, 
edge of Lake Racze, leg. H. Piotrowska 1954 (UGDA). AB32: Karsibórz, Kępa Karsiborska, pasture, leg. M. Wilhelm 2009 (SZUB). AB33: Trzciagowo, mossy meadow at bottom of chalk gully, leg. H. Piotrowska 1959 (UGDA); Trzciagowo, partly overgrown chalk pit, leg. M. Wilhelm 2012 (SZUB). AB34: Wolin, Dziwna river, meadow, leg. D. Żelewska 1992 (SZUB). AB62: Stolec, nature reserve "Świdwie", meadow and depression near a waterhole, E of village, leg. H. Więcław 2010 (**). AB64: Modrzewie, Bagna Krępskie, meadow, leg. H. Więcław 2008 (**). AC04: Stare Czarnowo, meadows near Lake Krzekno Wielkie, leg. F. Celiński 1961 (POZ). AC05: Dębina, meadows between village and Lake Miedwie, leg. F. Celiński 1962 (POZ); Grędziec, meadow near emergent vegetation at E edge of Lake Miedwie, leg. H. Więcław 2009 (**). AC16: Lubiatowo, N edge of Lake Torfowe, leg. H. Więcław 2009 (**); Lubiatowo, ditch and chalk pit near pasture, leg. B. Bosiacka 2009 (SZUB); Zaborsko, meadow at SE edge of Lake Zaborsko, leg. H. Więcław 2011 (**). AC25: Sitno, nature reserve "Tchórzyno", calcareous fen, leg. H. Więclaw $2010(* *)$. AC33: Trzcińsko, narrow strip of wet meadow between fish ponds and arable field, leg. B. Kurnicki 2012 (SZUB). AC35: Dąbrowa, meadow near Lake Myśliborskie, leg. H. Więcław 2012 (**); Mironów, Lake Chłop, meadow, leg. B. Kurnicki 2010 (SZUB). AC45: Myślibórz, edge of Lake Dalesz, leg. M. Wayda 2008 (KRA). AC47: edge of Lake Libenko, leg. M. Myśliwy 1999 (SZUB). BA91: $150 \mathrm{~m}$ W of Sianożęty, damp depression on cliff, leg. H. Więcław $2009(* *)$. BB00: Klasztorne Bagno, forest mire, leg. B. Bosiacka \& M. Myśliwy 2001 (SZUB). BB07: Jacinki, W of Polanów, mire, leg. J. Koopman 2011 (*). BB47: S of Drężno, meadow, leg. E. Kozioł 1975 (BIL). BB63: Nowe Worowo, wet meadow, leg. E. Kozioł 1973 (BIL). BB80: Studnica, E edge of Lake Kiełpino Duże, leg. H. Więcław 2007 (**); Ińsko, N edge of Lake Długie, leg. H. Więcław 2007 (**). BC13: Stawy Zawiślaka, fish ponds, leg. J. Kujawa-Pawlaczyk 1994 (DRAPN); moist edge of Lake Marta, leg. J. Kujawa-Pawlaczyk 1994 (DRAPN).

\section{Carex viridula var. pulchella}

Pomorskie. CA38: between Czarny Młyn and Ostrowo, depression between dunes, near road, usually flooded, leg. Z. Czubiński 1951 (POZ). CA59: Rewa, salt-marshes on Bay of Puck, leg. J. Kornaś 1959 (KRA). 
Appendix 2. Locality lists of hybrids within the section Ceratocystis

\section{Carex $\times$ alsatica Zahn $[$ C. demissa $\times$ C. flava $]$}

Dolnośląskie. BF47: Międzygórze, pasture near farmstay "Rancho u Bolka”, leg. H. Więcław 2011 (**).

Lódzkie. CE29: Ryś, alder carr, leg. Glynkiewicz 1975 (LOD). DE38: Piotrków, nature reserve "Wielkopole”, forest glade, leg. K. Żanet 1983 (LOD).

Małopolskie. DF78: Kraków-Podgórki Tynieckie, leg. W. Heise 2008 (KRA). DF98: Harbutowice, moist meadows in Gościbia stream valley, leg. B. Chwastowski 1977 (KRAM); nature reserve "Las Gościbia", Gościbka stream valley, fen in groundwater seepage area on SE slope, $460 \mathrm{~m}$ a.s.l., leg. H. \& T. Tacik 1965 (KRAM). DG28: E of Podwilk, hills in Pajakowy Wierch group, wet forest glade N of peak, $822 \mathrm{~m}$ a.s.l., leg. M. \& J. Guzikowie 1966 (KRAM). DG29: Odrowąż, wet meadow, $630 \mathrm{~m}$ a.s.l., leg. M. Guzikowa 1962 (KRAM). EF93: Żmiące-Zagnaniec, leg. M. Szewczyk 1994 (KRAM). EG02: Łopień, mire, leg. K. Towpasz 1967 (KRA). EG04: mountain range Jaworza, leg. J. Staszkiewicz 1966 (KRAM). EG29: Smerekowiec, fen in groundwater seepage area in forest, leg. W. Pisarek 1983 (LOD). DG70: Budzów, grassy border between fields, leg. D. Kempa 2006 (KRA). Mazowieckie. EC58: Łaś, moist meadow, leg. E. Rutkowska 1977 (WSRP). ED93: Różanna, riverine meadow, leg. A. Stańczak-Jażdżuk 1997 (LOD). EE24: Przysucha, moist forest road, leg. M. Nobis 2003 (KRA). EE34: Stefanków near Przysucha, moist meadow, leg. M. Nobis 2003 (KRA). EE35: Budki I near Szydłowiec, most meadow, leg. M. Nobis 2003 (KRA). FD30: Sawmill Prusy, fen in groundwater seepage area near railway tracks, leg. Z. Głowacki 1979 (WSRP).

Podkarpackie. FF16: Rędziny Sibigi, moist meadow, leg. A. Michalewska 2003 (KRA). FF24: Zalesie, waterlogged area in pine forest, leg. G. Dziundzio 1977 (KRA). FF27: Stary Jasiennik, moist meadow, leg. A. Michalewska 2004 (KRA). GG70: Wołosate, Przełęcz Beskid (mountain pass), roadside ditch, leg. H. Więcław 2010 (**).

Podlaskie. GB00: Pawłówka, peaty meadow, leg. S. Fieńko 1969 (BIL).

Pomorskie. CA90: 1.5 km S of Zielin, near road to Trzebielino, leg. E. Organiściak 1970 (POZ). CB23: Bytów, edge of Lake Lipusz, leg. W. Galikowska 1960 (POZ).

Śląskie. DE50: Kleśniska, alluvial forest, leg. M. Czernicka 1995 (LOD); Kleśniska, ditch edge, leg. J. Janiak 1998 (LOD). DE82: Gać near Blachownia, meadow, leg. J. Hereźniak 1972 (LOD); Aleksandria I, Herby Forest District, fen in groundwater seepage area in river valley near bridge, leg. J. Hereźniak 1975 (LOD). DF35: Trzebyczka, moist meadow, leg. B. BabczyńskaSendek $1988\left(^{*}\right)$; Dąbrowa Górnicza, meadow, leg. B. Babczyńska-Sendek 1988, $1989\left(^{*}\right)$. DG05: Okrajnik Stodałczyska, fen in groundwater seepage area in spruce forest, 410 m, leg. B. Kotońska 1986 (KRA). DG22: Olecki Górne, wet meadow, leg. M. Dudzik 1972 (LOD).

Świętokrzyskie. DE79: Osicyna near Brzeście, moist meadow, leg. M. Bielecki 2010 (KRA). EE45: Bliżyn near Kielce, fen near Bliżyński Reservoir, leg. M. Nobis 2003 (KRA). EE47: Trąbowiec, E part, drying emergent vegetation, leg. M. Nobis 2002 (KRA); 1.5 km SE of Zbijów Mały, peaty meadow, leg. M. Nobis 2002 (KRA). EE52: Smyków, moist thicket, leg. W. Paul 1990 (KRAM). EE57: Wąchock, moist meadow in Kamienna river valley, leg. M. Nobis 2002 (KRA). EE74: Kielce Podlesie, N of Góra Hałasa (mount), peaty meadow, leg. G. Łazarski 2013 (KRA). EE83: S of W end of village Brzeziny near Morawica, moist meadow, leg. G. Łazarski 2013 (KRA). EE84: SW of Suków-Borki, moist meadow, leg. G. Łazarski 2013 (KRA). EE85: Słopiec, about 20 km SE of Kielce, peatland, 250 m a.s.l., leg. J. Wójcicki 1974 (KRAM).

Warmińsko-Mazurskie. EB24: Pierwagi, most meadow, leg. L. Olesiński 1968 (OLTC). EB45: Rzeck, meadow on drained peatland, leg. L. Olesiński 1967 (OLTC).

Wielkopolskie. CD65: Gołuchów, forest, leg. M. Siepracha 1971 (LOD).

Zachodniopomorskie. BA91: Bagicz, meadow and roadside depression N of road Sianożęty-Bagicz, leg. H. Więcław 2011 $(* *)$. BC00: Kielpino near Choszczno, meadow in forest, leg. H. Więcław $2011(* *)$.

\section{Carex demissa $\times$ Carex viridula}

Mazowieckie. ED34: Podkowa Leśna, meadow, leg. K. Nowak 1971 (WA); Powsin, leg. H. Garbacik 1979 (WA). Podkarpackie: GG70: Wołosate, Przełęcz Beskid (mountain pass), roadside ditch, leg. H. Więcław 2010 (**).

\section{Carex $\times$ fulva Gooden. $[$ C. demissa $\times$ C. hostiana $]$}

Pomorskie. CA70: Świemirowo, leg. C. Lützow 1890 (TRN). CB29: Starogard, leg. C. Lützow 1894 (TRN). Warmińsko-Mazurskie. EB41: Jonkowo, moist peaty meadow, leg. L. Olesiński 1958 (OLTC).

\section{Carex $\times$ leutzii Kneuck. [C. hostiana $\times$ C. lepidocarpa]}

Pomorskie. CB22: Zapceń, small waterlogged site separated by a strip of mineral soil from Bagno Zapceń in direction of Kiedrowice, leg. S. Lisowski 1965 (POZ). 
Śląskie. DF02: Psary, N slope of Grojec (hill), moist meadow at base of limestone hill, leg. J. Hereźniak 1978 (LOD). Świętokrzyskie. EE82: S of E end of village Stawki near Polichno, moist meadow, leg. G. Łazarski 2013 (KRA). Warmińsko-Mazurskie. EB29: Ruciane, fen, leg. A. \& J. Kornaś 1952 (KRA).

\section{Carex $\times$ ruedtii Kneuck. [C. flava $\times$ C. lepidocarpa]}

Kujawsko-Pomorskie. CB78: Wałkowiska, fen near E edge of Lake Sierosławek, leg. H. Więcław 2009 (**). CB85: Piła Młyn, leg. M. Ceynowa-Giełdoń \& A. Siedlewska 1970 (TRN).

Lubelskie. GE02: nature reserve "Krowie Bagno" mire, leg. E. Hoteja \& St. Kucharzyk 1978 (KRAB); Wólka Wytycka, nature reserve "Krowie Bagno" mire, leg. B. Chwastowski 1978 (KRAM), leg. M. Szewczyk 1978 (KRAM); nature reserve "Brzeźno", leg. B. Chwastowski, 1978 (KRAM). GF21: Zamch, wet meadow in Wirowa river valley, leg. T. Krzaczek 1959 (LBLM).

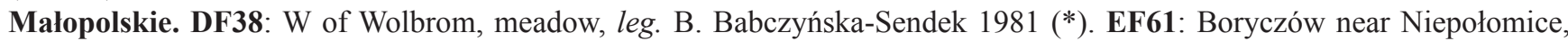
moist meadow, leg. J. Błaszczyk 1941 (KRA).

Mazowieckie. EE37: 2 km W of Pakosław, peaty meadow, leg. M. Nobis 2002 (KRA).

Podlaskie. FC98: Zajęczniki E, leg. P. Kalinowski 2013 (*). GB02: Berżniki, meadow in village, near Kunisjanka stream, leg. H. Więcław 2010 (**). GB11: Giby, moist meadow, leg. B. Sudnik 1975 (WA). GB63: Sterpejki, wet meadow, leg. B. Krawel 1982 (WSRP). GB94: Krynki, moist meadow, leg. A. Oleksza 1982 (WSRP).

Pomorskie. CA70: Słupsk, pond edge and meadow, leg. J. Smyl 1979 (SLTC).

Śląskie. DE91: Boronów, peaty meadow, leg. J. Hereźniak 1976 (LOD). DF35: Dąbrowa Górnicza-Strzemieszyce, fen in groundwater seepage area near railway tracks, leg. A. Błońska $2011(*)$.

Świętokrzyskie. EE65: Ciekoty, leg. Głowacka \& Lubańska 1961 (TRN). EE74: Kielce, waterlogged wasteland along a watercourse, leg. A. Ławik \& B. Maciejczak 1987 (KTC). FE61: Karczemka, fen, leg. R. Piwowarczyk 2002 (KRA).

Wielkopolskie. BC99: Dziewicza Góra (hill) near Poznań, wet meadows E of Miękówka, leg. W. Alkiewicz 1934 (POZ).

Zachodniopomorskie. AB64: Modrzewie, Bagna Krępskie (fen), meadow, leg. H. Więcław 2008 (**).

\section{Carex $\times$ schatzii Kneuck. [C. lepidocarpa $\times$ C. viridula $]$}

Małopolskie. DF36: Laski near Olkusz, Biała river valley, fen in groundwater seepage area, leg. H. Trzcińska-Tacik 1989 (KRA). DF37: Cieślin-Pustki, poor fen on Sączenica river, leg. B. Babczyńska-Sendek $1981\left(^{*}\right)$; Golczowice-Borek, at border

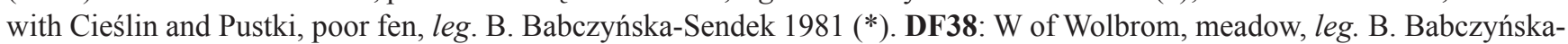
Sendek $1981(*)$.

Podlaskie. FA88: Jegliniszki, peaty meadow, leg. A. Sokołowski 1973 (BIL). FA97: Bachanowo, springhead near Czarna Hańcza river, leg. A. Sokołowski 1975 (BIL). GB30: Przewięź, mire, leg. A. Sokołowski 1972 (BIL). GC54: Skryplewo, wet meadow, leg. W. Bajko 1973 (BIL).

Pomorskie. CA59: Osłonino, low-sedge fen in nature reserve "Beka", S of village, leg. H. Więcław 2009 (**).

Śląskie. DF34: Dąbrowa Górnicza, Pogoria I, fen in groundwater seepage area, leg. A. Czylok 1995 (KTU); Dąbrowa Górnicza Antoniów, poor fen, leg. A. Błońska $2011(*)$.

Zachodniopomorskie. AC25: Sitno, calcareous fen near Lake Tchórzyno, leg. H. Więcław 2010 (*). AC35: Mironów, Lake Chłop, meadow, leg. B. Kurnicki 2010 (SZUB).

\section{Carex $\times$ subviridula Fernald $[$ C. flava $\times$ C. viridula $]$}

Lódzkie. DE14: Zbyszek, mire and meadow, leg. L. Kucharski \& W. Przybylińska 1978 (LOD). DE24: Chmielowiec, mire, leg. J. Hereźniak 1963 (LOD). DE35: Stefanowizna, wet meadow on Widawka river, leg. J. Hereźniak 1964 (LOD). DE41: Krzeczów near Kraszkowice, mire, leg. L. Fagasiewicz 1979 (LOD). DE45: Giżyzna, leg. J. Hereźniak 1964 (LOD). DE47: Kodrąb, meadow on Widawka river, leg. J. Hereźniak 1966 (LOD). DE49: Bąkowa Góra, fen, leg. P. Witosławski 1981 (LOD). DE59: Wojciechów, mire, leg. H. Leder 1971 (LOD). ED40: Serwitut near village Borowiny, clear-cut area, leg. M. Chabior 1997 (LOD). EE00: Tomaszów Mazowiecki, nature reserve "Niebieskie Źródła", meadow, leg. J. Mowszowicz \& R. Olaczek 1960 (LOD).

Małopolskie. DF38: W of Wolbrom, meadow, leg. B. Babczyńska-Sendek $1981\left(^{*}\right)$.

Mazowieckie. ED48: Całowanie, leg. J. Zielińska 1951 (WA). FC74: Garnek, leg. P. Kalinowski 2010 (*). FD07: Laskowice N, leg. P. Kalinowski 2013 (*), Kamianki-Czabaje, leg. P. Kalinowski 2013 (*); Góry NW, leg. P. Kalinowski 2013 (*).

Podlaskie. FB07: Jemieliste, wet birch forest on poor fen, leg. A. Pliszko 2010 (KRA). FC44: Ostrożne, meadow, leg. N. Romańczuk 1970 (BIL).

Śląskie. DE72: Kalej, peaty meadow, leg. J. Hereźniak 1972 (LOD). DE83: Częstochowa Błeszno, 32-70 Długa Street, moist meadow, leg. J. Hereźniak 1987 (LOD). DF25: Poręba near Zawiercie, poor fen, leg. A. Błońska 2009 (*).

Warmińsko-Mazurskie. DB59: Warlity Wielkie, drained bottom of former fish pond, leg. M. Środa 1997 (OLTC). 